Sobre a estrutura e a classificação

dos espaços sub-simétricos de codimensão maior que um.

Dulce Mary de Almeida

TESE APRESENTADA

AO

INSTITUTO DE MATEMÁTICA E ESTATÍSTICA

DA

UNIVERSIDADE DE SÃO PAULO

PARA OBTENÇÃO DO GRAU DE DOUTOR

EMI

MATEM.ÁTICA

Área de Concentração: Geometria diferencial

Orientador: Prof. Dr. Cláudio Gorodski

Co-orientador: Prof. Dr. José Antonio Verderesi

-São Paulo, abril de 2000- 


\section{Sobre a estrutura e a classificação dos espaços sub-simétricos de codimensão maior que um}

Este exemplar corresponde à redaçào final da tese devidamente corrigida defendida por Dulce Mary de Almeida e aprovada pela comissão julgadora.

São Paulo, 26 de abril de 2.000

Banca examinadora:

- Prof. Dr. José Antonio Verderesi (Presidente) - IME - USP

- Prof. Dr. Fabiano Gustavo Braga Brito - IME - USP

- Prof. Dr. Renato Hyuda de Luna Pedrosa - IMECC - UNICAMP

- Prof. Dr. Nikolai Alexandrovitch Goussevskii - UFMG

- Prof. Dr. José Miguel Martins Veloso - UFPA 


\section{Resumo}

O tema central desta tese é o estudo sobre a estrutura e a classificação dos espaços sub-simétricos de codimensão maior que um.

Este trabalho foi motivado pelo interesse em analisar, para codimensões mais altas, resultados de classificação existentes para espaços sub-simétricos munidos com distribuições de contato.

Entre os resultados originais obtidos, têm-se:

- Um teorema de existência e unicidade de uma conexão adaptada definida sobre uma variedade Riemanniana munida com distribuição. Neste contexto, apresenta-se também, uma lista de invariantes geométricos associados a esta conexão e um teorema de equivalência para variedades homogêneas adaptadas.

- A classificação completa dos espaços sub-simétricos simplesmente conexos equipados com distribuição de Engel. A metodologia utilizada para este fim consiste em linearizar a estrutura sub-Riemanniana através de uma classe especial de álgebras de Lie involutivas e investigar invariantes geométricos associados.

- A classificação dos espaços sub-simétricos simplesmente conexos, de dimensão sete, munidos de distribuição fortemente geradora por colchetes de codimensão três, que admitem um grupo de Lie de sub-isometrias, nilpotente. agindo transitivamente.

- Exemplos quaterniônicos de espaços sub-simétricos cuja distribuição associada é fortemente geradora por colchetes de codimensão três e alguns resultados pertinentes. 


\section{Abstract}

The main subject of this thesis is a study of the structure and classification of the subsymmetric spaces of codimension greather than one.

It was motivated by some previous results of classification for sub-symmetric spaces with contact distributions.

Some of the original results of this work are:

- An existence and uniqueness theorem for an adapted connection defined on a Riemannian manifold equipped with distributions. In this context, one also shows a list of geometrical invariants associated to this adapted connection. It is also proved one equivalence theorem for adapted homogeneous manifolds.

- The complete classification of the simply-connected sub-symmetric spaces equipped with the Engel distributions. The method used for this classification consists of linearizing the sub-Riemannian structure via a special class of involutive Lie algebras and investigating associated geometrical invariants.

- A classification of the simply-connected sub-symmetric spaces in dimension seven which admit a transitive nilpotent Lie group of sub-isometries and are equipped with fat distributions in codimension three.

- Some examples of quaternionic sub-symmetric spaces, whose associated distributions are fat, and some pertinent results. 
À minha màe. Cleusa, e ao meu filho, Heitor. Eu os amo imensamente e para sempre. 


\section{Agradecimentos}

Agradeço ao prof. Dr. Cláudio Gorodski pela sugestão do problema de tese e pela orientação.

Ao prof. Dr. José Antonio Verderesi pela co-orientação. Seria muito difícil concluir este trabalho sem sua ajuda.

A Daniel Tausk pela ajuda em diversos pontos deste trabalho.

A Gladys pela correção tipográfica.

A Alice pela tradução do resumo e pelas palavras de estímulo.

A meus irmãos Lucia, Luciana, Ricardo e Jean Carlo pelo carinho e pela torcida.

A memória de meu pai, Valdivino, pelo seu jeito torto de fazer as coisas certas.

A meu esposo, Jeo, pela compreensão. Não tenho palavras para agradecê-lo pela função dupla de pai e mãe. Que Deus vos abençoe por isso.

Aos amigos circunstanciais e não circunstanciais, do IME e extra IME, com quem partilhei um pouco de mim mesma nestes últimos cinco anos.

A todos que contribuiram direta ou indiretamente para a realização deste trabalho.

Ao $\mathrm{CNPq}$ pelo apoio financeiro.

A Deus pela minha vida e pelo muito que tem me dado. 


\section{Conteúdo}

Introdução 11

1 Variedades Riemannianas com distribuição 3

1.1 Conexão adaptada a uma variedade Riemanniana com distribuição . . . . . . . 3

1.2 Equivalência entre variedades homogêneas adaptadas . . . . . . . . . . i

1.3 Invariantes geométricos . . . . . . . . . . . . . . . . . 11

2 Espaços sub-simétricos com distribuição de Engel $\quad 14$

2.1 Distribuição de Engel . . . . . . . . . . . . . . . . . . . . . . 14

2.2 Espaços simétricos sub-Riemannianos de tipo Engel e álgebras de Lie involutivas associadas ......................... 16

2.3 A classificação dos espaços sub-simétricos com distribuição de Engel . . . . . 20

3 Estrutura dos espaços sub-simétricos com distribuição fortemente geradora $\begin{array}{ll}\text { por colchetes } & 29\end{array}$

3.1 Distribuição fortemente geradora por colchetes . . . . . . . . . . . . . . . 29

3.2 Métrica associada a uma variedade sub-Riemanniana com distribuição fortemente geradora por colchetes . . . . . . . . . . . . . . . . . 30

3.3 Espaços sub-simétricos e álgebras de Lie involutivas sub-ortogonais associadas 3.5

3.4 Métrica canônica no caso sub-simétrico . . . . . . . . . . . . . . . . . 40

3.5 Sub-torções no caso sub-simétrico . . . . . . . . . . . . . . . . 43

4 Espaços sub-simétricos de codimensão três com distribuição fortemente geradora por colchetes $\quad 45$

4.1 Classificação dos espaços sub-simétricos em dimensão sete com grupo de isometrias nilpotente . . . . . . . . . . . . . . . . . . . 45

4.2 Exemplos quaterniônicos . . . . . . . . . . . . . . . . . . . 49

$\begin{array}{ll}\text { Bibliografia } & 61\end{array}$ 


\section{Introdução}

O objetivo deste trabalho é investigar a estrutura e a classificação dos espaços simétricos sub-Riemannianos equipados com distribuição geradora por colchetes de codimensão maior que um.

Este trabalho nasceu do interesse em analisar, para codimensòes mais altas, o comportamento de resultados de classificação existentes para o caso de distribuição de contato, uma vez que neste caso a distribuição em questão tem necessariamente codimensão um. Mais especificamente, foi motivado pela seguinte seqüência de resultados:

$(1.986,[10])$ Strichartz classificou completamente os espaços simétricos sub-Riemannianos de contato de dimensão três.

(1.996, [3]) Falbel e Gorodski ampliaram a classificação dada por Strichartz aos espaços homogêneos sub-Riemannianos de contato de dimensão três e aos espaços sub-simétricos de contato ímpar de dimensão quatro.

(1.995-8, [1], [2], [4]) Bieliavsky, Falbel, Gorodski e Rumin classificaram completamente os espaços simétricos sub-Riemannianos de contato.

No primeiro capítulo. demonstramos a existência e unicidade de uma conexão definida sobre uma variedade Riemanniana com distribuição e adaptada à distribuição. Esta conexão pode ser considerada como uma generalização da conexão adaptada definida em ([1], [5]) para variedades sub-Riemannianas equipadas com distribuição de contato. Listamos também, alguns invariantes geométricos associados a esta conexão e provamos um teorema de equivalência para variedades homogêneas adaptadas. Convém enfatizar que este capítulo tem um caráter bastante abrangente, uma vez que se aplica a todas as variedades sub-Riemannianas cuja métrica sub-Riemanniana possui uma extensão Riemanniana intrínsica.

No capítulo 2, apresentamos a classificação completa dos espaços simétricos sub-Riemannianos $(M, \mathcal{D}, g)$, simplesmente conexos, 4-dimensionais, com distribuição $\mathcal{D}$ regular e geradora por colchetes de codimensão dois. Neste caso, necessariamente, $\mathcal{D}$ possui vetor de crescimento $(2,3,4)$, isto é, para todo ponto $p \in M$ tem-se $\operatorname{dim}\left(\mathcal{D}^{2}\right)_{p}=3 \mathrm{e}\left(\mathcal{D}^{3}\right)_{p}=T_{p} M$ 
onde $\left(\mathcal{D}^{2}\right)_{p}$ é o subespaço de $T_{p} M$ gerado por todos os colchetes de seções de $\mathcal{D}$ avaliados no ponto $p$ e $\left(\mathcal{D}^{3}\right)_{p}$ é o subespaço de $T_{p} M$ gerado por todos os colchetes do tipo $[X,[Y, Z]]_{p}$ com $X, Y$ e $Z$ seções de $\mathcal{D}$. Uma distribuição $\mathcal{D}$ com estas propriedades é denominada distribuição de Engel. Sob estas hipóteses provamos que $M$ é um grupo de Lie $G$ com estrutura sub-Riemanniana invariante à esquerda $(\mathcal{D}, g)$ e cuja álgebra de Lie é involutiva, e elaboramos uma lista completa de possibilidades para $G, \mathcal{D}$ e $g$. Para distinguir dentre as possíveis distribuições $\mathcal{D}$ e métricas $g$ aquelas que originavam estruturas sub-Riemannians equivalentes, encontramos um paralelismo definido sobre uma variedade sub-Riemanniana arbitrária com estrutura de tipo Engel, isso nos permitiu associar invariantes geométricos. Finalmente, os resultados obtidos da investigação desses invariantes no caso de um espaço sub-simétrico tornou possível fechar completamente a referida classificação.

No terceiro capítulo, definimos distribuições fortemente geradoras por colchetes e observamos que esta é a generalização natural de distribuições de contato. Apresentamos para variedades sub-Riemannianas munidas com este tipo de distribuição a construçào de uma métrica Riemanniana, que estende canônicamente a métrica sub-Riemanniana. É importante destacar que esta construção se encontra em ([13], seção 7$)$, e os resultados aqui apresentados correspondem apenas a uma reinterpretaçào um pouco mais minuciosa. Provamos também um teorema de estrutura para espaços sub-simétricos cuja distribuição associada é deste tipo, via álgebras de Lie involutivas sub-ortogonais e utilizamos esta estrutura para caracterizar, no caso sub-simétrico, a métrica Riemanniana canônica e os invariantes definidos no primeiro capítulo.

Finalmente, no quarto e último capítulo, apresentamos alguns exemplos de espaços subsimétricos $(M . \mathcal{D}, g)$ equipados com distribuições fortemente geradoras por colchetes de codimensào três. A primeira classe de exemplos é obtida em dimensão sete, com a hipótese adicional da existência de um grupo de Lie nilpotente de sub-isometrias agindo diferenciavelmente e transitivamente em $M$. Neste contexto, provamos que $M$ é um grupo de Lie cuja álgebra de Lie possui uma estrutura involutiva bastante particular, e esta particularidade tornou possivel a classificação neste caso. Os demais exemplos correspondem a uma $S p(1)$ fibração sobre espaços simétricos Riemannianos equipados com estrutura quaterniônica e foram motivados pela técnica de se construir espaços sub-simétricos de contato, simplesmente conexos, irredutíveis, como $S^{1}$-fibração sobre espaços simétricos Hermitianos irredutíveis. 


\section{Capítulo 1}

\section{Variedades Riemannianas com distribuição}

Neste capítulo demonstraremos a existência e unicidade de uma conexão adaptada a uma variedade Riemanniana com distribuição e apresentaremos alguns invariantes geométricos associados. Provaremos também um teorema de equivalência para variedades homogêneas adaptadas.

\subsection{Conexão adaptada a uma variedade Riemanniana com distribuição}

No que segue, $(M, \mathcal{D},<\cdot, \cdot>)$ denota uma variedade Riemanniana munida de uma distribuição $\mathcal{D}$ (ie, $\mathcal{D}$ é um subfibrado do fibrado tangente $T M$ ) de dimensão $n$ e codimensão $d$ e $<\cdot, \cdot>$ é uma métrica Riemanniana definida em $M$.

Seja $\mathcal{D}^{\perp}$ a distribuição em $M$ que a cada ponto $x \in M$ associa o complemento ortogonal à $\mathcal{D}_{x}$ em $T_{x} M$. A métrica Riemannina $<\cdot, \cdot>$ definida sobre $M$, possibilita definir de modo natural e de maneira única uma conexão sobre $M$ adaptada a $\mathcal{D}$. Esta conexão, em analogia com a conexão Levi-Cività da geometria Riemanniana, é uma conexão com torção (em geral não nula) que preserva as distribuições $\mathcal{D}$ e $\mathcal{D}^{\perp}$ e é compatível com a métrica.

Mais precisamente, seja $(M, \mathcal{D},<\cdot, \cdot>)$ uma variedade Riemanniana com distribuição e sejam $\underline{T M}, \underline{\mathcal{D}}$ e $\underline{\mathcal{D}}^{\perp}$ o conjunto das seções de $T M, \mathcal{D}$ e $\mathcal{D}^{\perp}$ respectivamente, então

Teorema 1.1 Existe uma única conexão $\nabla: \underline{T M} \rightarrow \underline{T M^{*}} \odot \underline{T M}$, denominada a conexão adaptada, e unicamente dois tensores $\tau: \mathcal{D}^{\perp} \otimes \mathcal{D} \rightarrow \mathcal{D}$ e $\sigma: \mathcal{D} \odot \mathcal{D}^{\perp} \rightarrow \mathcal{D}^{\perp}$ simétricos na segunda variável, denominados as sub-torções, com as seguintes propriedades ( $T$ é o tensor torção da conexão): 
a. $\quad \nabla_{U}: \underline{\mathcal{D}} \rightarrow \underline{\mathcal{D}} \quad, \quad \nabla_{U^{\prime}}: \underline{\mathcal{D}}^{\perp} \rightarrow \underline{\mathcal{D}}^{\perp} ;$

b. $\nabla<\cdot, \cdot>=0$;

c. $T(\xi, X)=\tau_{\xi}(X)+\sigma_{X}(\xi)$,

$$
T(X, Y)=-\left[X, Y^{\perp}\right. \text {, }
$$$$
T(\xi, \eta)=-[\xi, \eta]^{\top} \text {. }
$$

para todo $X, Y \in \underline{\mathcal{D}} ; \xi, \eta \in \underline{\mathcal{D}}^{\perp} \in U \in \underline{T M}$, onde $\left[X, Y^{\perp}\right]^{\perp}$ denota a projeção de $[X, Y]$ em $\mathcal{D}^{\perp} e[\xi, \eta]^{\top}$ denota a projeção de $[\xi, \eta]$ em $\mathcal{D}$.

\section{Prova:}

Sejam $X, Y, Z \in \underline{\mathcal{D}}$ e $\xi, \eta, \zeta \in \underline{\mathcal{D}}^{\perp}$. Em analogia com a conexào Levi-Cività, $\nabla_{X} Y$ e $\nabla_{\eta} \xi$ são unicamente determinados usando a.. b., c. e a permutaçào cíclica. da seguinte maneira:

$$
\begin{aligned}
& X<Y, Z>+Y<Z, X>-Z<X, Y>= \\
& 2<\nabla_{X} Y, Z>+<Y \cdot[X, Z]+T(X, Z)> \\
& +<X,[Y, Z]+T(Y, Z)>+<Z,[Y, X]+T(Y, X)> \\
& =2<\nabla_{X} Y, Z>+<Y,[X, Z]^{\top}>+<X,[Y, Z]^{\top}>+<Z,[Y, X]^{\top}> \\
& \begin{aligned}
\eta< & \xi, \zeta>+\xi<\zeta, \eta>-\zeta<\eta, \xi>= \\
& 2<\nabla_{\eta} \xi, \zeta>+<\xi,[\eta \cdot \zeta]+T(\eta, \zeta)> \\
& +<\eta,[\xi, \zeta]+T(\zeta, \xi)>+<\zeta,[\xi, \eta]+T(\xi, \eta)> \\
& =2<\nabla_{\eta} \xi, \zeta>+<\xi,[\eta, \zeta]^{\perp}>+<\eta,[\xi, \zeta]^{\perp}>+<\zeta,[\xi, \eta]^{\perp}>
\end{aligned}
\end{aligned}
$$

Para definir $\nabla_{\xi} X$ e $\nabla_{X} \xi$, lembramos que $\nabla_{\xi} X-\nabla_{X} \xi=\left[\xi, X^{\prime}\right]+T(\xi, X)$, portanto

$$
\begin{aligned}
\nabla_{\xi} X^{\top} & =\left(\nabla_{\xi} X^{\top}-\nabla_{X} \xi\right)^{\top} \\
& =\left(\left[\xi, X^{\top}\right]+T(\xi, X)\right)^{\top}=\left[\xi, X^{\top}+\tau_{\xi}\left(X^{\top}\right)\right. \\
\nabla_{X} \xi & =-\left(\nabla_{\xi} X-\nabla_{X} \xi\right)^{\perp} \\
& =-\left(\left[\xi, X^{\top}\right]+T(\xi, X)\right)^{\perp}=-\left[\xi, X^{\perp}-\sigma_{X}(\xi)\right.
\end{aligned}
$$

Finalmente,

$$
\begin{aligned}
\xi<X, Y>= & <\nabla_{\xi} X, Y>+<X, \nabla_{\xi} Y> \\
= & <\left[\xi, X^{\top}+\tau_{\xi}(X), Y>+<X,[\xi, Y]^{\top}+\tau_{\xi}(Y)>\right. \\
& =2<\tau_{\xi}(X), Y>+<\left[\xi, X^{\top}\right]^{\top}, Y>+<[\xi, Y]^{\top}, X>
\end{aligned}
$$




$$
\begin{aligned}
X<\xi, \eta> & =<\nabla_{X} \xi, \eta>+<\xi, \Gamma_{X} \eta> \\
& =<-\left[\xi, X^{\perp}\right]^{\perp}-\sigma_{X}(\xi), \eta>+<\xi,-[\eta, X]^{\perp}-\sigma_{X}(\eta)> \\
& =-2<\sigma_{X}(\xi), \eta>-<[\xi, X]^{\perp}, \eta>-<\left[\eta, X^{\perp}, \xi>\right.
\end{aligned}
$$

determinam $\tau_{\xi}\left(X^{\prime}\right)$ e $\sigma_{X}(\xi)$ unicamente.

Observação 1.1 Se $\bar{\nabla}$ denota a conexão Levi-Cività relativa à métrica $\langle\cdot$,$\rangle . então$ $\nabla_{X} Y=\left(\bar{\nabla}_{X} Y\right)^{\top}$ e $\nabla_{\xi} \zeta=\left(\bar{\nabla}_{\xi} \zeta\right)^{\perp} \forall X, Y \in \underline{\mathcal{D}} ; \forall \xi, \zeta \in \underline{\mathcal{D}^{\perp}}$ (onde $\left(\bar{\nabla}_{X} Y\right)^{\top}$ denota a projeção de $\bar{\nabla}_{X} Y$ em $\mathcal{D}$ e $\left(\bar{\nabla}_{\xi} \zeta\right)^{\perp}$ denota a projeção de $\bar{\nabla}_{\xi} \zeta$ em $\left.\mathcal{D}^{\perp}\right)$.

No teorema seguinte daremos uma versão local do teorema anterior no contexto de formas. Por simplicidade de notação, a partir deste momento fica implícito (salvo mençào ao contrário) que os índices latinos $i, j, s$ variam de 1 a $n$ e os índices gregos $\alpha, \beta, \gamma$ variam de $n+1$ a $n+d$.

Teorema 1.2 Seja $(M, \mathcal{D},<\cdot, \cdot>)$ uma variedade Riemanniana com distribuição e sejam $\left\{E_{i}, E_{\alpha}\right\}$ referencial ortonormal local adaptado, definido em $U \subset M$ (ie $\left\{E_{i}\right\}$ é um referencial ortonormal de $\mathcal{D}$ e $\left\{E_{\alpha}\right\}$ é um referencial ortonormal de $\left.\mathcal{D}^{\perp}\right)$ e $\left\{\theta^{i}, \theta^{\alpha}\right\}$ o co-referencial dual associado. Então existe um único conjunto de l-formas $\left\{\omega_{i}^{j}, \omega_{\alpha}^{\beta}, \tau_{\alpha}^{i}, \sigma_{i}^{\alpha}\right\}$ definidas $\epsilon m U$, satisfazendo as equações:

$$
\begin{aligned}
& d \theta^{i}=\sum_{j=1}^{n} \theta^{j} \wedge \omega_{j}^{i}+\sum_{\alpha=n+1}^{n+d} \theta^{\alpha} \wedge \tau_{\alpha}^{i}+\sum_{n+1 \leq \alpha<\beta \leq n+d} \theta^{i}\left(\left[E_{\beta}, E_{\alpha}\right]\right) \theta^{\alpha} \wedge \theta^{3} \\
& d \theta^{\alpha}=\sum_{\beta=n+1}^{n+d} \theta^{\beta} \wedge \omega_{\beta}^{\alpha}+\sum_{i=1}^{n} \sigma_{i}^{\alpha} \wedge \theta^{i}+\sum_{1 \leq i<j \leq n} \quad \theta^{\alpha}\left(\left[E_{j}, E_{i}\right]\right) \theta^{i} \wedge \theta^{j}
\end{aligned}
$$

com condiçòes

$$
\begin{aligned}
& \text { i) } \omega_{j}^{i}=-\omega_{i}^{j} \\
& \text { ii) } \omega_{\beta}^{\alpha}=-\omega_{\alpha}^{\beta} \\
& \text { iii) } \sum_{i=1}^{n} \tau_{\alpha}^{i} \wedge \theta^{i}=0 \\
& \text { iv) } \sum_{\alpha=n+1}^{n+d} \sigma_{i}^{\alpha} \wedge \theta^{\alpha}=0
\end{aligned}
$$




\section{Prova:}

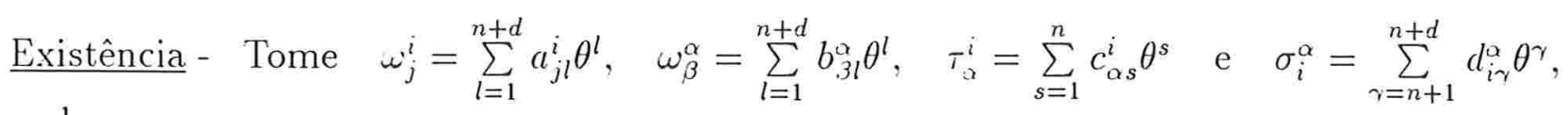
onde:

$$
\begin{aligned}
& a_{j s}^{i}=\frac{1}{2}\left(\theta^{i}\left[E_{s}, E_{j}\right]+\theta^{j}\left[E_{i}, E_{s}\right]+\theta^{s}\left[E_{i}, E_{j}\right]\right) \\
& a_{j \gamma}^{i}=\frac{1}{2}\left(\theta^{i}\left[E_{\gamma}, E_{j}\right]-\theta^{j}\left[E_{\gamma}, E_{i}\right]\right) \\
& b_{3 \gamma}^{\alpha}=\frac{1}{2}\left(\theta^{\alpha}\left[E_{\gamma}, E_{\beta}\right]+\theta^{\beta}\left[E_{\alpha}, E_{\gamma}\right]+\theta^{\gamma}\left[E_{\alpha}, E_{\beta}\right]\right) \\
& b_{3 s}^{\alpha}=\frac{1}{2}\left(\theta^{\alpha}\left[E_{s}, E_{\beta}\right]-\theta^{\beta}\left[E_{s}, E_{\alpha}\right]\right) \\
& c_{\alpha s}^{i}=-\frac{1}{2}\left(\theta^{i}\left[E_{\alpha}, E_{s}\right]+\theta^{s}\left[E_{\alpha}, E_{i}\right]\right) \\
& d_{i \gamma}^{\alpha}=\frac{1}{2}\left(\theta^{\alpha}\left[E_{i}, E_{\gamma}\right]+\theta^{\gamma}\left[E_{i}, E_{\alpha}\right]\right)
\end{aligned}
$$

Unicidade - Seja $\left\{\omega_{i}^{j}, \omega_{\alpha}^{\beta}, \tau_{\alpha}^{i}, \sigma_{i}^{\alpha}\right\}$ qualquer conjunto de 1-formas definidas em $U$, satisfazendo as condições enunciadas. Se $\left\{\tilde{\omega}_{i}^{j}, \tilde{\omega}_{\alpha}^{\beta}, \tilde{\tau}_{\alpha}^{i}, \tilde{\sigma}_{i}^{\alpha}\right\}$ também satisfaz as mesmas condições, então:

$$
\begin{array}{r}
\sum_{j=1}^{n} \theta^{j} \wedge\left(\omega_{j}^{i}-\tilde{\omega}_{j}^{i}\right)+\sum_{\alpha=n+1}^{n+d} \theta^{\alpha} \wedge\left(\tau_{\alpha}^{i}-\tilde{\tau}_{\alpha}^{i}\right)=0 \\
\sum_{\beta=n+1}^{n+d} \theta^{\beta} \wedge\left(\omega_{\beta}^{\alpha}-\tilde{\omega}_{\beta}^{\alpha}\right)+\sum_{i=1}^{n}\left(\sigma_{i}^{\alpha}-\tilde{\sigma}_{i}^{\alpha}\right) \wedge \theta^{i}=0 \\
\sum_{i=1}^{n}\left(\tau_{\alpha}^{i}-\tilde{\tau}_{\alpha}^{i}\right) \wedge \theta^{i}=0 \\
\sum_{\alpha=n+1}^{n+d}\left(\sigma_{i}^{\alpha}-\tilde{\sigma}_{i}^{\alpha}\right) \wedge \theta^{\alpha}=0 \\
\left(\omega_{j}^{i}-\tilde{\omega}_{j}^{i}\right)=-\left(\omega_{i}^{j}-\tilde{\omega}_{i}^{j}\right) \\
\left(\omega_{\beta}^{\alpha}-\tilde{\omega}_{\beta}^{\alpha}\right)=-\left(\omega_{\alpha}^{\beta}-\tilde{\omega}_{\alpha}^{\beta}\right)
\end{array}
$$

Aplicando o Lema de Cartan a equação (1.1) obtemos (para $i, j=1, \ldots, n ; \alpha=n+1, \ldots, n+d)$ :

$$
\begin{aligned}
& \omega_{j}^{i}-\tilde{\omega_{j}^{i}}=\sum_{k=1}^{n+d} a_{j k}^{i} \theta^{k} \\
& \tau_{\alpha}^{i}-\tilde{\tau_{\alpha}^{i}}=\sum_{k=1}^{n+d} a_{\alpha k}^{i} \theta^{k} \\
& a_{l k}^{i}=a_{k l}^{i} \\
& \text { para } l, k=1,2, \ldots, n+d \text {. }
\end{aligned}
$$


Da equação (1.5) segue que: $a_{j k}^{i}=-a_{i k}^{j}$ para $k=1.2 \ldots, n+d$. Assim:

$$
a_{j k}^{i}=-a_{i k}^{j}=-a_{k i}^{j}=a_{j i}^{k}=a_{i j}^{k}=-a_{k j}^{i}=-a_{j k}^{i} \quad \text { para } \quad k=1,2, \ldots, n .
$$

Portanto, $a_{j}^{i} k=0$ para $i . j, k=1,2, \ldots, n$. Novamente, aplicando o Lema de Cartan a equação (1.3) temos $(\forall a$ e $\forall i)$ :

$$
\tau_{\alpha}^{i}-\tilde{\tau_{\alpha}^{i}}=\sum_{s=1}^{n} b_{\alpha s}^{i} \theta^{s} \quad \operatorname{com} \quad b_{\alpha s}^{i}=b_{\alpha i}^{s} \quad \text { para } \quad i, s=1,2, \ldots, n .
$$

Então, $a_{\alpha k}^{i}=0$ para $k=n+1, n+2, \ldots, n+d$ e $a_{\alpha k}^{i}=b_{\alpha k}^{i}$ para $k=1,2, \ldots, n$. Assim,

$$
a_{\alpha k}^{i}=b_{\alpha k}^{i}=b_{\alpha i}^{k}=a_{\alpha i}^{k}=a_{i \alpha}^{k}=-a_{k \alpha}^{i}=-a_{\alpha k}^{i} \quad \text { para } k=1,2, \ldots, n .
$$

Logo, $a_{\alpha k}^{i}=0$ para $k=1,2, \ldots, n$ e como $a_{\alpha k}^{i}=a_{k \alpha}^{i}$ temos também que $a_{j k}^{i}=0$ para $k=n+1, n+2, \ldots, n+d$.

Provamos então a unicidade das 1 -formas $\omega_{j}^{i}$ e $\tau_{\alpha}^{i}$. De forma análoga, usando as equações $(1.2),(1.4)$ e (1.6) e o Lema de Cartan temos a unicidade das $\omega_{\alpha}^{\beta}$ e $\sigma_{i}^{\alpha}$.

Observação 1.2 Note que. se $\left\{E_{i}, E_{\alpha}\right\}$ é um referencial ortonormal local adaptado definido em $U \subset M$, então em $U$ podemos escrever : $\nabla E_{i}=\sum_{j=1}^{n} \omega_{i}^{j} \otimes E_{j}, \quad \nabla E_{\alpha}=\sum_{\beta=n+1}^{n+d} \omega_{\alpha}^{\beta} \otimes E_{\beta}$, $\tau_{E_{\alpha}}=\sum_{j=1}^{n} \tau_{\alpha}^{j} \odot E_{j} \quad$ e $\quad \sigma_{E_{i}}=\sum_{\alpha=n+1}^{n+d} \sigma_{i}^{\alpha} \odot E_{\alpha}$.

Definição 1.1 A curvatura desta conexão é dada por:

$$
R(X, Y) Z=\nabla_{X} \nabla_{Y} Z-\nabla_{Y} \nabla_{X} Z-\nabla_{[X, Y]} Z \quad \forall X, Y, Z \in \underline{T M}
$$

\subsection{Equivalência entre variedades homogêneas adap- tadas}

Uma isometria adaptada local entre variedades com distribuição $(M, \mathcal{D},<\cdot,>>)$ e $\left(M^{\prime}, \mathcal{D}^{\prime}\right.$, $\left.<\cdot, \cdot\rangle^{\prime}\right)$ é um difeomorfismo entre conjuntos abertos $\psi: U \subset M \rightarrow U^{\prime} \subset M^{\prime}$ tal que $\left.\psi^{*}<\cdot, \cdot\right\rangle^{\prime}=\langle\cdot, \cdot\rangle\left(\psi^{\prime}\right.$ é isometria local Riemanniana) e $\psi_{*}(\mathcal{D})=\mathcal{D}^{\prime}(\psi$ preserva a distribuição). Se $\psi$ está globalmente definida diremos apenas que $\psi$ é uma isometria adaptada. 
Um espaço homogêneo adaptado é uma variedade Riemanniana com distribuição $(M . \mathcal{D}$, $<\cdot, \cdot\rangle$ ) que admite um grupo de Lie de isometrias adaptadas agindo diferenciavelmente e transitivamente em $M$.

A seguir apresentaremos um teorema de equivalência entre variedades homogêneas adaptadas.

Teorema 1.3 Sejam $(M . \mathcal{D},<\cdot, \cdot>) e\left(M^{\prime}, \mathcal{D}^{\prime},<\cdot, \cdot>^{\prime}\right)$ variedades homogêneas adaptadas, com $M$ simplesmente conexa. Sejam $T, R$ (respectivamente $T^{\prime}, R^{\prime}$ ) os tensores torçào $e$ curvatura de $M$ (repectivamente $M^{\prime}$ ). Se $x_{0} \in M, x_{0}^{\prime} \in M^{\prime}$ e se $l: T_{x_{0}} M \rightarrow T_{x_{0}^{\prime}} M^{\prime}$ $\dot{\epsilon}$ uma isometria linear que leva $\mathcal{D}_{x_{0}}$ em $\mathcal{D}_{x_{0}^{\prime}}^{\prime}$ e os tensores $T_{x_{0}}$ e $R_{x_{0}}$ nos tensores $T_{x_{0}^{\prime}}^{\prime} e$ $R_{x_{0}^{\prime}}^{\prime}$, respectivamente, então existe $f: M \rightarrow M^{\prime}$ isometria adaptada tal que $f\left(x_{0}\right)=x_{0}^{\prime}$ e $(d f)_{x_{0}}=l$.

\section{Prova:}

Sejam $R(M)$ e $R\left(M^{\prime}\right)$ os fibrados de referenciais adaptados relativos à $M$ e $M^{\prime}$, respectivamente. Denotemos por $(\theta, \omega)$ e $\left(\theta^{\prime}, \omega^{\prime}\right)$ as respectivas forma fundamental e forma de conexão associadas a $R(M)$ e $R\left(M^{\prime}\right)$. Consideremos a variedade produto $R(M) \times R\left(M^{\prime}\right)$, sejam $\pi: R(M) \times R\left(M^{\prime}\right) \rightarrow R(M)$ e $\pi^{\prime}: R(M) \times R\left(M^{\prime}\right) \rightarrow R\left(M^{\prime}\right)$ as projeções. Consideremos agora as 1 -formas $\alpha, \beta$ definidas sobre $R(M) \times R\left(M^{\prime}\right)$ por:

$$
\begin{aligned}
& \alpha=\pi^{*} \theta-\left(\pi^{\prime}\right)^{*} \theta^{\prime} \\
& \beta=\pi^{*} \omega-\left(\pi^{\prime}\right)^{*} \omega^{\prime}
\end{aligned}
$$

Analisemos as condições de integrabilidade do sistema diferencial $\{$ a. 3$\}$ em $R(M) \times R\left(M^{\prime}\right)$. Denotemos por $\mathcal{T}$ e $\Omega$ (resp. $\mathcal{T}^{\prime}$ e $\Omega^{\prime}$ ) os tensores torçào e curvatura de $\omega$ em $R(M)$ (resp. $u^{\prime}$ em $\left.R\left(M^{\prime}\right)\right)$. Das equaçòes de estrutura temos:

$$
\left\{\begin{array} { r l } 
{ d \theta + \omega \wedge \theta } & { = \mathcal { T } } \\
{ d \omega + \omega \wedge \omega } & { = \Omega }
\end{array} \quad \text { } \quad \left\{\begin{array}{l}
d \theta^{\prime}+\omega^{\prime} \wedge \theta^{\prime}=\mathcal{T}^{\prime} \\
d \omega^{\prime}+\omega^{\prime} \wedge \omega^{\prime}=\Omega^{\prime}
\end{array}\right.\right.
$$

Assim, lembrando que a diferencial (das formas fundamental e de conexão) e o produto exterior comutam com o "pull back" (das projeções), temos:

$$
\begin{aligned}
d \alpha & =\pi^{*} d \theta-\left(\pi^{\prime}\right)^{*} d \theta^{\prime} \\
& =\pi^{*}(\mathcal{T}-\omega \wedge \theta)-\left(\pi^{\prime}\right)^{*}\left(\mathcal{T}^{\prime}-\omega^{\prime} \wedge \theta^{\prime}\right) \\
& =\pi^{*} \mathcal{T}-\left(\pi^{\prime}\right)^{*} \mathcal{T}^{\prime}-\pi^{*} \omega \wedge \pi^{*} \theta+\left(\pi^{\prime}\right)^{*} \omega^{\prime} \wedge\left(\pi^{\prime}\right)^{*} \theta^{\prime}
\end{aligned}
$$




$$
\begin{aligned}
& =\pi^{*} \mathcal{T}-\left(\pi^{\prime}\right)^{*} \mathcal{T}^{\prime}-\pi^{*} \omega \wedge \pi^{*} \theta+\left(\pi^{\prime}\right)^{*} \omega^{\prime} \wedge\left(\pi^{\prime}\right)^{*} \theta^{\prime}+\pi^{*} \omega \wedge\left(\pi^{\prime}\right)^{*} \theta^{\prime}-\pi^{*} \omega \wedge\left(\pi^{\prime}\right)^{*} \theta^{\prime} \\
& =\pi^{*} \mathcal{T}-\left(\pi^{\prime}\right)^{*} \mathcal{T}^{\prime}-\pi^{*} \omega \wedge\left(\pi^{*} \theta-\left(\pi^{\prime}\right)^{*} \theta^{\prime}\right)-\left(\pi^{*} \omega-\left(\pi^{\prime}\right)^{*} \omega^{\prime}\right) \wedge\left(\pi^{\prime}\right)^{*} \theta^{\prime} \\
& =\pi^{*} \mathcal{T}-\left(\pi^{\prime}\right)^{*} \mathcal{T}^{\prime}-\pi^{*} \omega \wedge \alpha+\left(\pi^{\prime}\right)^{*} \theta^{\prime} \wedge 3 \\
& d \beta=\pi^{*} d \omega-\left(\pi^{\prime}\right)^{*} d \omega^{\prime} \\
& =\pi^{*}(\Omega-\omega \wedge \omega)-\left(\pi^{\prime}\right)^{*}\left(\Omega^{\prime}-\omega^{\prime} \wedge \omega^{\prime}\right) \\
& =\pi^{*} \Omega-\left(\pi^{\prime}\right)^{*} \Omega^{\prime}-\pi^{*} \omega \wedge \pi^{*} \omega+\left(\pi^{\prime}\right)^{*} \omega^{\prime} \wedge\left(\pi^{\prime}\right)^{*} \omega^{\prime} \\
& =\pi^{*} \Omega-\left(\pi^{\prime}\right)^{*} \Omega^{\prime}-\pi^{*} \omega \wedge \pi^{*} \omega+\left(\pi^{\prime}\right)^{*} \omega^{\prime} \wedge\left(\pi^{\prime}\right)^{*} \omega^{\prime}+\pi^{*} \omega \wedge\left(\pi^{\prime}\right)^{*} \omega^{\prime}-\pi^{*} \omega \wedge\left(\pi^{\prime}\right)^{*} \omega^{\prime} \\
& =\pi^{*} \Omega-\left(\pi^{\prime}\right)^{*} \Omega^{\prime}-\pi^{*} \omega \wedge\left(\pi^{*} \omega-\left(\pi^{\prime}\right)^{*} \omega^{\prime}\right)-\left(\pi^{*} \omega-\left(\pi^{\prime}\right)^{*} \omega^{\prime}\right) \wedge\left(\pi^{\prime}\right)^{*} \omega^{\prime} \\
& =\pi^{*} \Omega-\left(\pi^{\prime}\right)^{*} \Omega^{\prime}-\pi^{*} \omega \wedge \beta+\left(\pi^{\prime}\right)^{*} \omega^{\prime} \wedge \beta
\end{aligned}
$$

Portanto, para concluir que o sistema é involutivo precisamos mostrar que $\left(\pi^{*} \mathcal{T}-\left(\pi^{\prime}\right)^{*} \mathcal{T}^{\prime}\right)$ e $\left(\pi^{*} \Omega-\left(\pi^{\prime}\right)^{*} \Omega^{\prime}\right)$ estão no ideal gerado por a e $\beta$. Lembremos que:

$$
\begin{array}{llll}
\theta: T R(M) & \rightarrow \mathbb{R}^{n+d}, & \theta=\left(\theta^{i}\right) \\
\omega: T R(M) & \rightarrow \mathfrak{o}(n) \times \mathfrak{o}(d) \subset \mathfrak{g l}(n+d, I R), & \omega=\left(\omega_{j}^{i}\right) \\
\mathcal{T}: \wedge^{2} T R(M) \rightarrow \mathbb{R}^{n+d}, & \mathcal{T}=\left(T^{i}\right) \\
\Omega: \wedge^{2} T R(M) \rightarrow \mathfrak{o}(n) \times \mathfrak{o}(d) \subset \mathfrak{g l}(n+d, I R), & \Omega=\left(\Omega_{j}^{i}\right)
\end{array}
$$

As formas $\left(\theta^{i}, \omega_{j}^{i}\right)$ definem um paralelismo em $R(M)$. Visto que a torção e a curvatura são horizontais, temos:

$$
\begin{aligned}
T^{i} & =\sum_{j, k=1}^{n+d} A_{j k}^{i} \theta^{j} \wedge \theta^{k} \\
\Omega_{j}^{i} & =\sum_{k, l=1}^{n+d} R_{j k l}^{i} \theta^{k} \wedge \theta^{l}
\end{aligned}
$$

onde $A_{j k}^{i}$ e $R_{j k l}^{i}$ são funções em $R(M)$ 
Agora, $R_{g}{ }^{*} \cdot T=g^{-1} \mathcal{T}$ onde $R_{g}: R(M) \rightarrow R(M)$ é a translação a direita por um elemento $g \in O(n) \times O(d)$. implica que:

$$
\mathcal{T}_{\epsilon \cdot g}\left(d R_{3}(u), d R_{g}(v)\right)=g^{-1}\left(\mathcal{T}_{e}(u, v)\right) \quad \forall e \in R(M) ; \forall u, v \in T_{e} R(M)
$$

portanto,

$$
\sum_{j, k=1}^{n+d} A_{j k}^{i}(e . g)\left(R_{g}{ }^{*} \theta^{j}\right) \wedge\left(R_{g}{ }^{*} \theta^{k}\right)(u, v)=\sum_{j, k, s=1}^{n+d}\left(g^{-1}\right)_{s}^{i} A_{j k}^{s}(e)\left(\theta^{j} \wedge \theta^{k}\right)(u, v)
$$

Como $R_{g}{ }^{\times} \theta=g^{-1} \theta$, vem que:

$$
\text { para } i=1 \ldots . n+d \text {. }
$$

$$
\sum_{j, k=1}^{n+d} \sum_{l, r=1}^{n+d} A_{j k}^{i}(e . g)\left(g^{-1}\right)_{l}^{j}\left(g^{-1}\right)_{r}^{k}\left(\theta^{l} \wedge \theta^{r}\right)(u, v)=\sum_{l, r, s=1}^{n+d}\left(g^{-1}\right)_{s}^{i} A_{l r}^{s}(e)\left(\theta^{l} \wedge \theta^{r}\right)(u, v)
$$

Segue que:

$$
\sum_{j, k=1}^{n+d} A_{j k}^{i}(e . g)\left(g^{-1}\right)_{l}^{j}\left(g^{-1}\right)_{r}^{k}=\sum_{s=1}^{n+d}\left(g^{-1}\right)_{s}^{i} A_{l r}^{s}(e)
$$

De onde para $i, l, r=1, \ldots, n+d$.

$$
A_{j k}^{i}(e . g)=\sum_{l, r=1}^{n+d}\left\{\sum_{s=1}^{n+d}\left(g^{-1}\right)_{s}^{i} A_{l r}^{s}(\epsilon)\right\} g_{j}^{l} g_{k}^{r}
$$

Assim,

para $i, j, k=1 \ldots, n+d$.

$$
T^{i}(e . g)=\sum_{j, k=1}^{n+d} \sum_{l, r=1}^{n+d}\left\{\sum_{s=1}^{n+d}\left(g^{-1}\right)_{s}^{i} A_{l r}^{s}(e)\right\} g_{j}^{l} g_{k}^{r} \theta_{e . g}^{j} \wedge \theta_{e . g}^{k}
$$

$$
\text { para } i=1, \ldots, n+d \text {. }
$$

Abreviaremos por:

$$
T_{e . g}=g^{-1} A(e)(g \theta)_{e . g} \wedge(g \theta)_{e . g}
$$

Por hipótese $M$ é uma variedade homogênea adaptada. Assim, para cada $x \in M$ existe uma isometria adaptada $f^{x}: M \rightarrow M$ tal que $f^{x}\left(x_{0}\right)=x$, onde $x_{0} \in M$ está fixado. Fixemos em 
$x_{0}$ um referencial adaptado $e_{x_{0}}=\left(e_{1}, \ldots, e_{n+d}\right)$ de $R(M)$ e consideremos a seção de $R(. M)$ definida por:

$$
\epsilon(x)=\left(x, d f_{x_{0}}^{x}\left(e_{1}\right), \ldots, d f_{x_{0}}^{x}\left(\epsilon_{n+d}\right)\right)
$$

Como isometria preserva a torção, temos:

$$
\left(e^{*} \mathcal{T}\right)_{x}=\left(e^{*} T^{i}\right)_{x}=\sum_{j, k=1}^{n+d} A_{j k}^{i}(e(x)) e^{*} \theta^{j} \wedge e^{*} \theta^{k} \quad \text { com } A_{j k}^{i}(\epsilon(x))=\text { constante }=A
$$

Então, $\left(\pi^{*} \mathcal{T}\right)_{e(x) \cdot g}=g^{-1} A\left[g \pi^{*} \theta \wedge g \pi^{*} \theta\right]$.

Considerando que $M^{\prime}$ é outra variedade homogênea adaptada, pelo mesmo argumento, também temos: $\left[\left(\pi^{\prime}\right)^{*} \mathcal{T}^{\prime}\right]_{e^{\prime}(x) \cdot g}=g^{-1} A^{\prime}\left[g\left(\pi^{\prime}\right)^{*} \theta^{\prime} \wedge g\left(\pi^{\prime}\right)^{*} \theta^{\prime}\right]$.

Agora, podemos escolher $e_{x_{0}}=\left(e_{1}, . ., e_{n+d}\right)$ e $e_{x_{0}}{ }^{\prime}=\left(\epsilon_{1}^{\prime}=l\left(e_{1}\right), \ldots, e_{n+d}^{\prime}=l\left(\epsilon_{n+d}\right)\right)$ de maneira que $A=A^{\prime}$, pois a função linear $l: T_{x_{0}} M \rightarrow T_{x_{0}^{\prime}} \cdot M^{\prime}$ transporta a torção de $M$ na torção de $M^{\prime}$. Então temos:

$$
\begin{aligned}
\left(\pi^{*} \mathcal{T}\right)_{e(x) \cdot g}-\left(\left(\pi^{\prime}\right)^{*} \mathcal{T}^{\prime}\right)_{e^{\prime}(x) \cdot g} & =g^{-1} A\left\{g \pi^{*} \theta \wedge g \pi^{*} \theta-g\left(\pi^{\prime}\right)^{*} \theta^{\prime} \wedge g\left(\pi^{\prime}\right)^{*} \theta^{\prime}\right\} \\
& =g^{-1} A\left\{\left[g \pi^{*} \theta-g\left(\pi^{\prime}\right)^{*} \theta^{\prime}\right] \wedge\left[g \pi^{*} \theta+g\left(\pi^{\prime}\right)^{*} \theta^{\prime}\right]\right\} \\
& =g^{-1} A g\left\{\alpha \wedge\left[g \pi^{*} \theta+g\left(\pi^{\prime}\right)^{*} \theta^{\prime}\right]\right\}
\end{aligned}
$$

Analogamente fazemos o mesmo raciocínio para a curvatura. Logo o sistema é integrável. Então, dados $\left(e, e^{\prime}\right) \in R(M) \times R\left(M^{\prime}\right)$ existe uma única variedade conexa maximal, que passa por $\left(e, e^{\prime}\right)$, integral a $\{\alpha, \beta\}$, dada pelo Teorema de Frobenius. Esta subvariedade integral é dada pelo gráfico de uma função $F: R(M) \rightarrow R\left(M^{\prime}\right)$, definida apenas localmente, a qual se projeta numa funçào $f: U \subset M \rightarrow U^{\prime} \subset M^{\prime}$, que é uma isometria local adaptada. Agora, $M, M^{\prime}$ são homogêneas, portanto completas, $M$ é simplesmente conexa, então, por argumento de analiticidade $f$ se prolonga a uma isometria global.

\subsection{Invariantes geométricos}

Associaremos alguns invariantes geométricos, como segue.

Para cada $x \in M$ consideremos o operador bilinear anti-simétrico $b: \mathcal{D}_{x} \times \mathcal{D}_{x} \rightarrow \mathcal{D}_{x}^{\perp}$, definido por

$$
b(\mathrm{v}, \mathrm{w})=\left([\mathrm{V}, \mathrm{W}]_{x}\right)^{\perp} .
$$


onde $V$. W sào extensòes de $v$. w à seçòes de $\mathcal{D}$. Assim. cada $\xi \in \mathcal{D}_{x}^{\perp}$ induz uma 1-forma bilinear anti-simétrica a valores reais $b_{\xi}: \mathcal{D}_{x} \times \mathcal{D}_{x} \rightarrow \mathbb{R}$, dada por

$$
b_{\xi}(v, w)=<b(v, w), \xi>_{x} .
$$

Portanto, existe um único operador linear anti-simétrico $A_{\xi}: \mathcal{D}_{x} \rightarrow \mathcal{D}_{x}$ tal que

$$
b_{\xi}(\mathrm{v}, \mathrm{w})=<A_{\xi}(\mathrm{v}), \mathrm{w}>_{x}
$$

Denotamos por $\operatorname{End}\left(\mathcal{D}_{x}^{\perp}\right)$ o conjunto de todos os endomorfismo de $\mathcal{D}_{x}^{\perp}$ e definimos o operador bilinear $\bar{b}: \mathcal{D}_{x} \times \mathcal{D}_{x} \rightarrow \operatorname{End}\left(\mathcal{D}_{x}^{\perp}\right) . \bar{b}(\mathrm{v}, \mathrm{w})=\bar{b}_{\mathrm{vw}}: \mathcal{D}_{x}^{\perp} \rightarrow \mathcal{D}_{x}^{\perp}$ dado por

$$
\bar{b}_{\mathrm{vw}}(\xi)=b\left(A_{\xi}(\mathrm{v}), \mathrm{w}\right) \text {. }
$$

Observamos que o operador linear

$$
\hat{b}=\operatorname{traço} \bar{b}=\sum_{i=1}^{n} \bar{b}_{e_{i} e_{i}}: \mathcal{D}_{x}^{\perp} \rightarrow \mathcal{D}_{x}^{\perp}
$$

onde $\beta=\left\{e_{1}, e_{2}, \ldots, e_{n}\right\}$ é uma base ortonormal de $\mathcal{D}_{x}$, independe da base $\beta$ escolhida e é um operador linear simétrico. Este fato nos possibilita definir os invariantes $\lambda_{n+1}, \ldots, \lambda_{n+d}$ auto-valores associados ao operador $\hat{b}$.

Analogamente, cada $v \in \mathcal{D}_{x}$ induz uma 1-forma bilinear anti-simétrica a valores reais $t_{\mathrm{v}}: D_{x}{ }^{\perp} \times \mathcal{D}_{x}{ }^{\perp} \rightarrow \mathbb{R}$,

$$
t_{v}(\xi, \eta)=<t(\xi, \eta), v>_{x}
$$

onde $t: \mathcal{D}_{x}^{\perp} \times \mathcal{D}_{x}^{\perp} \rightarrow \mathcal{D}_{x}$ é dado por

$$
t(\xi, \eta)=\left([\tilde{\xi}, \tilde{\eta}]_{x}\right)^{\top}
$$

onde $\tilde{\xi} ; \tilde{\eta}$ são extensões de $\xi, \eta$ à seções de $\mathcal{D}^{\perp}$. Assim, denotando por $\operatorname{End}\left(\mathcal{D}_{x}\right)$ o conjunto de todos os endomorfismos de $\mathcal{D}_{x}$. temos um operador bilinear $\bar{t}: \mathcal{D}_{x}{ }^{\perp} \times \mathcal{D}_{x}{ }^{\perp} \rightarrow \operatorname{End}\left(\mathcal{D}_{x}\right)$, $\bar{t}(\xi, \eta)=\bar{t}_{\xi \eta}: \mathcal{D}_{x} \rightarrow \mathcal{D}_{x}$ definido por

$$
\bar{t}_{\xi \eta}(\mathrm{v})=\mathrm{t}\left(\mathrm{S}_{\mathrm{v}}(\xi), \eta\right)
$$

onde $S_{\mathrm{v}}: \mathcal{D}_{x}{ }^{\perp} \rightarrow \mathcal{D}_{x}^{\perp}$ é o único operador linear anti-simétrico tal que

$$
<S_{\mathrm{v}}(\xi), \eta>_{x}=t_{\mathrm{v}}(\xi, \eta)
$$

O operador linear

$$
\hat{t}=\operatorname{traço} \bar{t}=\sum_{\alpha=n+1}^{n+d} \bar{t}_{e_{\alpha} e_{\alpha}}: \mathcal{D}_{x} \rightarrow \mathcal{D}_{x}
$$


onde $\gamma=\left\{e_{n+1}, e_{n+2}, \ldots . \epsilon_{n+d}\right\}$ é uma base ortonormal de $\mathcal{D}_{x}{ }^{\perp}$, independe da base $;$ escolhida e é um operador linear simétrico. Assim, podemos definir os invariantes $\lambda_{1}, \ldots . \lambda_{n}$ auto-valores associados ao operador $\hat{t}$.

Por outro lado, os operadores bilineares:

$$
\begin{aligned}
& A: \mathcal{D}_{x}^{\perp} \times \mathcal{D}_{x}^{\perp} \longrightarrow \operatorname{End}\left(\mathcal{D}_{x}\right) \\
& (\xi, \eta) \longmapsto A_{\xi} \circ A_{\eta} \\
& S: \mathcal{D}_{x} \times \mathcal{D}_{x} \longrightarrow \operatorname{End}\left(\mathcal{D}_{x}^{\perp}\right) \\
& (\mathrm{v}, \mathrm{w}) \longmapsto S_{\mathrm{v}} \circ S_{\mathrm{w}}
\end{aligned}
$$

induzem. respectivamente, os operadores lineares simétricos:

$$
\begin{aligned}
& \hat{A}=\operatorname{traço~} A=\sum_{\alpha=n+1}^{n+d} A\left(e_{\alpha}, e_{\alpha}\right)=\sum_{\alpha=n+1}^{n+d}\left(A_{e_{\alpha}} \circ A_{e_{\alpha}}\right): \mathcal{D}_{x} \rightarrow \mathcal{D}_{x} \\
& \hat{S}=\operatorname{traço~} S=\sum_{i=1}^{n} S\left(e_{i}, e_{i}\right)=\sum_{i=1}^{n}\left(S_{e_{i}} \circ S_{e_{i}}\right): \mathcal{D}_{x}^{\perp} \rightarrow \mathcal{D}_{x}^{\perp}
\end{aligned}
$$

onde $\left\{\epsilon_{1}, \ldots, e_{n+d}\right\}$ é qualquer base ortonormal adaptada de $T_{x} M$, e nos fornece os invariantes $\rho_{i}(i=1 \ldots, n)$ e $\rho_{\alpha}(\alpha=n+1, \ldots, n+d)$ auto-valores associados aos operadores $\hat{A}$ e $\hat{S}$.

Temos também os invariantes, definidos através dos tensores $\tau$ e $\sigma$, da seguinte maneira. Consideramos os operadores bilineares:

$$
\begin{aligned}
& \bar{\tau}: \mathcal{D}_{x}^{\perp} \times \mathcal{D}_{x}^{\perp} \longrightarrow \operatorname{End}\left(\mathcal{D}_{x}\right) \\
& (\xi, \eta) \longmapsto \tau_{\xi} \circ \tau_{\eta} \\
& \bar{\sigma}: \mathcal{D}_{x} \times \mathcal{D}_{x} \longrightarrow \operatorname{End}\left(\mathcal{D}_{x}^{\perp}\right) \\
& (\mathrm{v}, \mathrm{w}) \longmapsto \sigma_{\mathrm{v}} \circ T_{\mathrm{w}}
\end{aligned}
$$

Definimos os operadores lineares:

$$
\begin{aligned}
& \hat{\tau}=\operatorname{traço} \bar{\tau}=\sum_{\alpha=n+1}^{n+d} \bar{\tau}\left(e_{\alpha}, e_{\alpha}\right)=\sum_{\alpha=n+1}^{n+d}\left(\tau_{e_{\alpha}} \circ \tau_{e_{\alpha}}\right): \mathcal{D}_{x} \rightarrow \mathcal{D}_{x} \\
& \hat{\sigma}=\operatorname{traço~} \bar{\sigma}=\sum_{i=1}^{n} \bar{\sigma}\left(e_{i}, e_{i}\right)=\sum_{i=1}^{n}\left(\sigma_{e_{i}} \circ \sigma_{e_{i}}\right): \mathcal{D}_{x}^{\perp} \rightarrow \mathcal{D}_{x}^{\perp}
\end{aligned}
$$

Temos que $\hat{\tau}$ e $\hat{\sigma}$ são operadores lineares simétricos, bem definidos e nos permitem definir os invariantes $\widehat{\tau}_{1}, \ldots, \widehat{\tau}_{n}$ e $\widehat{\sigma}_{n+1}, \ldots, \widehat{\sigma}_{n+d}$, auto-valores associados aos operadores $\hat{\tau}$ e $\hat{\sigma}$, respectivamente.

Evidentemente, os vetores

$$
\begin{aligned}
& H_{\tau}=\operatorname{traço~} \tau_{x}=\sum_{\alpha=n+1}^{n+d}\left(\text { traço } \tau_{e_{\alpha}}\right) e_{\alpha} \in \mathcal{D}_{x}{ }^{\perp} \\
& H_{\sigma}=\operatorname{traço~} \sigma_{x}=\sum_{i=1}^{n}\left(\operatorname{traço~} \sigma_{e_{i}}\right) e_{i} \in \mathcal{D}_{x}
\end{aligned}
$$

também são invariantes. 


\section{Capítulo 2}

\section{Espaços sub-simétricos com distribuição de Engel}

Uma variedade sub-Riemanniana é uma terna $(M, \mathcal{D}, g)$ onde $M$ é uma variedade diferenciável, $\mathcal{D}$ é uma distribuicão sobre $M$ (ie, $\mathcal{D}$ é um subfibrado vetorial próprio do fibrado tangente $T M$ ) e $g$ é uma métrica diferenciável definida em $\mathcal{D}$.

Neste capítulo restringiremos nossa atenção às variedades sub-Riemannianas orientadas cuja distribuição associada é uma distribuição de Engel orientada. Obteremos um paralelismo sobre uma estrutura sub-Riemanniana de tipo Engel com a finalidade de definir invariantes geométricos. e então classificaremos todas as variedades sub-Riemannianas 4 dimensionais simplesmente conexas, as quais são sub-simétricas, usando uma linearizaçào da estrutura via uma classe especial de álgebras de Lie involutivas.

\subsection{Distribuição de Engel}

Seja $T_{x} M$ o espaço tangente no ponto $x \in M$. Dizemos que a distribuição $\mathcal{D}$ é geradora por colchetes se para qualquer $x \in M$ as seções locais de $\mathcal{D}$ juntamente com todos os seus colchetes iterados avaliados no ponto $x$ geram o espaço vetorial $T_{x} M$.

Seja $\left(\mathcal{D}^{2}\right)_{x}$ o subespaço de $T_{x} M$ gerado por todos os colchetes de seções de $\mathcal{D}$ avaliados no ponto $x$. Denote-se por $\left(\mathcal{D}^{3}\right)_{x}$ o subespaço de $T_{x} M$ gerado por todos os campos vetoriais do tipo $[[X, Y], Z]_{x}$, onde $X, Y, Z$ variam sobre todas as seções de $\mathcal{D}$.

Neste capítulo consideraremos somente o caso em que $\mathcal{D}$ é uma distribuição de Engel. Isto significa que $\mathcal{D}$ é uma distribuição de codimensão dois em uma variedade 4-dimensional.$M$. regular e geradora por colchetes, ou equivalentemente $\mathcal{D}$ é uma distribuição de codimensão dois em uma variedade $M$ de dimensào 4 e para cada $x \in M$ tem-se $\operatorname{dim}\left(\mathcal{D}^{2}\right)_{x}=3 \mathrm{e}$ $\left(\mathcal{D}^{3}\right)_{x}=T_{x} M$. 
Denotemos por $\mathcal{D}^{2}$ a distribuiçào definida sobre $M$, que associa a cada $x \in M$ o subespaço $\left(\mathcal{D}^{2}\right)_{x}$ de $T_{x}$, I. Consideremos a forma de Levi de $\mathcal{D}^{2}$

$$
\mathcal{L}: \mathcal{D}^{2} \times \mathcal{D}^{2} \rightarrow T M / \mathcal{D}^{2}, \quad \mathcal{L}_{x}(v, w)=[V, W]_{x} \quad \bmod \mathcal{D}_{x}^{2}
$$

onde $V, W$ são extensões de $v, w$ a seções de $\mathcal{D}^{2}$. Temos o seguinte lema.

Lema 2.1 Seja $\mathcal{D}$ uma distribuição de Engel e $\mathcal{L}$ a forma de Levi de $\mathcal{D}^{2}$, então $\mathcal{L}$ é uma forma bilinear anti-simétrica sobre $\mathcal{D}^{2}$, seu núcleo é 1-dimensional $\epsilon$ está contido $\in m \mathcal{D}$.

\section{Prova:}

É claro que $\mathcal{D} \subset \mathcal{D}^{2}$, pois dado $X, Y$ seções de $\mathcal{D}$ passando por $x \in M$, podemos localmente encontrar uma funçào $f$, definida numa vizinhança aberta de $x$ a valores reais. tal que, $d f_{x}\left(Y_{x}\right)=1$. Desta forma, $\left[Y, f X^{\prime}\right]_{x}=X_{x}+f(x)\left[Y, X_{x}\right.$, portanto $X_{x} \in \mathcal{D}_{x}^{2}$. Como $\operatorname{dim}$ $T_{x} M=4$ e $\operatorname{dim} \mathcal{D}_{x}^{2}=3$ temos que $T_{x} M / \mathcal{D}_{x} \cong \mathbb{R}$, e esta identificaçào junto com a antisimetria do colchete de Lie nos dizem que $\mathcal{L}$ é uma forma bilinear anti-simétrica sobre $\mathcal{D}^{2}$. Assim, posto de $\mathcal{L}$ é par, logo as únicas possibilidades são posto de $\mathcal{L}$ igual a zero ou posto de $\mathcal{L}$ igual a dois. Mas, posto de $\mathcal{L}=0$ não pode ocorrer, pois neste caso o núcleo de $\mathcal{L}$ coincide com $\mathcal{D}^{2}$, e isto contraria a hipótese $\left[\mathcal{D}^{2}, \mathcal{D}\right]=T M$, imposta na definição de distribuição de Engel. Portanto, posto de $\mathcal{L}$ é igual a dois e consequentemente o núcleo de $\mathcal{L}$ é 1-dimensional. Vamos mostrar agora que o núcleo de $\mathcal{L}$ está contido em $\mathcal{D}$. Seja $\xi$ no núcleo de $\mathcal{L}$, então em particular $\xi \in \mathcal{D}^{2}$ e assim podemos expressar $\xi$ localmente por $\xi=a X+b Y+c[X, Y]$ onde $X, Y$ são seçòes locais de $\mathcal{D}$ num aberto $U$, tais que $\mathcal{D}_{x}$ é gerado por $\left\{X_{x}, Y_{x}\right\} \forall x \in U$, e $a, b, c$ são funções $\mathcal{C}^{\infty}$ definidas em $U$ a valores reais. Agora.

$$
\begin{gathered}
{[\xi, X]_{x}=-d a_{x}\left(X_{x}\right) X_{x}-d b_{x}\left(X_{x}\right) Y_{x}+\left(b(x)+d c_{x}\left(X_{x}\right)\right)\left[Y . X^{\prime}\right]_{x}+c(x)\left[[X, Y], X^{\prime}\right]_{x}} \\
{[\xi, Y]_{x}=-d a_{x}\left(Y_{x}\right) X_{x}-d b_{x}\left(Y_{x}\right) Y_{x}+\left(a(x)-d c_{x}\left(Y_{x}\right)\right)[X . Y]_{x}+c(x)[[X, Y], Y]_{x} .}
\end{gathered}
$$

Como $\{X, Y,[X, Y]\}$ é um referencial local de $\mathcal{D}^{2}$, segue que $\xi$ pertence ao núcleo de $\mathcal{L}$ se, e somente se, $\forall x \in U$ tem-se: i) $c(x)=0$, ou ii) $\left[[X . Y], X^{\prime}\right]_{x}$ e $\left.[X, Y], Y\right]_{x} \in \mathcal{D}_{x}^{2}$. Porém, novamente da hipótese $\left[\mathcal{D}^{2}, \mathcal{D}\right]=T M$, temos que $i i$ ) não pode ocorrer. Portanto, em $U$ temos $\xi=a X+b Y$, ou seja $\xi \in \mathcal{D}$.

A partir de agora admitiremos (salvo menção explícita em contrário) $M$ e $\mathcal{D}$ orientadas, nestas condições segue do Lema [2.1] a existência de uma estrutura Riemanniana canônica 
sobre $(M, \mathcal{D}, g)$, determinada como segue: escolhemos um campo vetorial unitário $Y_{2} \in \mathcal{D}$, tal que $Y_{2}$ gera o núcleo de $\mathcal{L}$ (observe que nestas condições existem somente duas escolhas possíveis para $Y_{2}$ ); escolhido $Y_{2}$ consideremos $Y_{1}$ como o único campo vetorial unitário sobre $\mathcal{D}$ tal que $\left\{Y_{1}, Y_{2}\right\}$ é um referencial ortonormal positivo sobre $\mathcal{D}$. Definimos $\xi_{3}=\left[Y_{1}, Y_{2}\right]$, $\xi_{4}=\left[Y_{1}, \xi_{3}\right]$ e estendemos $g$ a uma métrica Riemanniana $\langle\cdot, \cdot\rangle$ sobre $M$, impondo $\xi_{3}, \xi_{4}$ ortonormais à $\mathcal{D}$. Eliminamos a ambiguidade na escolha de $Y_{2}$ exigindo que $\left\{Y_{1}, Y_{2}, \xi_{3}, \xi_{4}\right\}$, construido sob as condiçòes acima. seja um referencial ortonormal positivo sobre $M$.

O referencial ortonormal positivo $\left\{Y_{1}, Y_{2}, \xi_{3}, \xi_{4}\right\}$ definido acima é denominado referencial canônico associado a variedade sub-Riemanniana $(M, \mathcal{D}, g)$.

\subsection{Espaços simétricos sub-Riemannianos de tipo En- gel e álgebras de Lie involutivas associadas}

Uma isometria local sub-Riemanniana entre duas variedades sub-Riemannianas $(M, \mathcal{D}, g)$ e $\left(M^{\prime}, \mathcal{D}^{\prime}, g^{\prime}\right)$ é um difeomorfismo entre conjuntos abertos $\psi: U \subset M \rightarrow U^{\prime} \subset M^{\prime}$ tal que $\psi_{*}(\mathcal{D})=\mathcal{D}^{\prime}$ e $\psi^{*} g^{\prime}=g$. No caso em que $\mathcal{D}$ é uma distribuição de Engel, $\psi$ é uma isometria Riemanniana local relativa à métrica Riemanniana estendida sobre $M$ e $M^{\prime}$ (uma vez que $\psi_{*} Y_{1}= \pm Y_{1}^{\prime}, \psi_{*} Y_{2}= \pm Y_{2}^{\prime}$ e $\left.\psi_{*}[\cdot, \cdot]=\left[\psi_{*} \cdot, \psi_{*} \cdot\right]\right)$. Se $\psi$ está globalmente definida de $M$ em $M^{\prime}$, diremos simplesmente que $\psi$ é uma isometria sub-Riemanniana.

Um espaço homogêneo sub-Riemanniano é uma variedade sub-Riemanniana $(M, \mathcal{D}, g)$ que admite um grupo de Lie transitivo de isometrias sub-Riemannianas agindo diferenciavelmente em $M$.

Um espaço simétrico sub-Riemanniano (ou espaço sub-simétrico) é uma variedade subRiemanniana $(M, \mathcal{D}, g)$ tal que, para cada ponto $x_{0} \in M$ existe uma isometria sub-Riemanniana $\psi$, chamada sub-simetria em $x_{0}$, satisfazendo $\psi\left(x_{0}\right)=x_{0}$ e $\left.\psi_{*}\right|_{\mathcal{D}_{x_{0}}}=-I d$. Ressaltamos que esta é uma definição simplificada, que se aplica ao caso das distribuições particulares consideradas neste trabalho: distribuições de Engel e distribuições fortemente geradoras por colchetes (estudadas nos capítulos 3 e 4). A título de informação, mencionamos agora, a definição de espaços sub-simétricos, dada em geral.

Definição 2.1 (Strichartz [10]) l'm espaço simétrico sub-Riemanniano é uma variedade sub-Riemanniana $(M, \mathcal{D}, g)$ que admite um grupo de Lie de isometrias sub-Riemannianas, agindo diferenciavelmente e transitivamente em $\mathrm{M}$, com as seguintes propriedades:

i) O subgrupo de isotropia $\mathrm{K}$ de um ponto $x_{0}$ é compacto. 
ii) Is contém um elemento $\psi$ tal que $\left.d \psi_{x_{0}}\right|_{\mathcal{D}_{x_{0}}}=-I d$ e $\psi^{2}=I d$.

Observamos que se as propriedades i) e ii) valem em um ponto, então da homogeneidade, elas se verificam em todos os pontos. No caso das distribuições de Engel, o subgrupo de isotropia $K$ de um ponto $x_{0}$ é finito, portanto compacto, e o quadrado da sub-simetria em $x_{0}, \psi^{2}$, é igual a identidade, uma vez que, neste caso, uma isometria sub-Riemanniana de $M$, fixa (a menos de sinais) o referencial canônico.

O lema seguinte e seu corolário justificam a omissão da hipótese de homogeneidade na definição simplificada de espaços sub-simétricos. Nos casos particulares das distribuições estudadas neste trabalho, a homogeneidade decorre da existência da sub-simetria em cada ponto.

Lema 2.2 Seja $(M, \mathcal{D},<\cdot, \cdot>)$ variedade Riemanniana com $\mathcal{D}$ distribuição geradora por colchetes $\in \Gamma$ sua conexão adaptada, então quaisquer dois pontos de $M$ podem ser ligados por uma $\Gamma$-geodésica horizontal quebrada.

\section{Prova:}

Análoga a demonstração do Lema (1.1) de [1]

Corolário 2.1 Seja $(M, \mathcal{D}, g)$ uma variedade sub-Riemanniana, com $\mathcal{D}$ distribuiçào de Engel, tal que para cada ponto $x_{0} \in M$ existe uma isometria sub-Riemanniana $\psi: M \rightarrow M$ satisfazendo $\psi\left(x_{0}\right)=\left.x_{0} e \psi_{*}\right|_{\mathcal{D}_{x_{0}}}=-I d$. Então $(M, \mathcal{D}, g)$ é um espaço homogéneo subRiemanniano.

\section{Prova:}

Seja $\gamma:[0.1] \rightarrow M$ uma $\nabla$-geodésica horizontal, então a sub-simetria em $\gamma(t / 2)$ leva $\gamma(0)$ em $\gamma(t)$. Como do Lema [2.2] quaisquer dois pontos podem ser ligados por uma $\nabla$-geodésica quebrada. o resultado segue.

Uma álgebra de Lie involutiva é uma álgebra de Lie real g equipada com um automorfismo $s: \mathfrak{g} \rightarrow \mathfrak{g}$ satisfazendo $s^{2}=1$ ( $s$ é chamada uma involução da álgebra de Lie $\mathfrak{g}$ ). É claro que se $s$ é uma involução da álgebra de Lie $\mathfrak{g}$ então $\mathfrak{g}=\mathfrak{g}^{+} \oplus \mathfrak{g}^{-}$onde $\mathfrak{g}^{+}=\left\{X \in \mathfrak{g} \mid s\left(X^{+}\right)=X\right\}$, $\mathfrak{g}^{-}=\left\{X \in \mathfrak{g} \mid s(X)=-X^{\prime}\right\}$. 
O próximo teorema e seu corolário mostram que um espaço homogêneo sub-Riemanniano de tipo Engel pode ser identificado com um grupo de Lie $G$, o qual possui uma estrutura subRiemanniana invariante à esquerda $(\mathcal{D}, g)$ e cuja álgebra de Lie $\mathfrak{g}$ possui uma decomposição especial; em particular, se o espaço é sub-simétrico então g é involutiva.

Teorema 2.1 Seja $(M, \mathcal{D}, g)$ um espaço homogêneo sub-Riemanniano simplesmente conexo 4-dimensional, onde $\mathcal{D}$ é uma distribuição de Engel. Então:

1. a componente conexa contendo a identidade do grupo de todas as isometrias subRiemannianas de. $\mathrm{I}$, denotado por $G$, é um grupo de Lie, simplesmente conexo. que age transitivamente e simplesmente sobre $M$;

2. a álgebra de Lie $\mathfrak{g}$ de $G$ possui uma decomposição $\mathfrak{g}=\mathfrak{p}+[\mathfrak{p}, \mathfrak{p}]+[[\mathfrak{p}, \mathfrak{p}] . \mathfrak{p}]$, onde $\mathfrak{p}$ corresponde à $\mathcal{D}_{x_{0}}$ através da identificação de $\mathfrak{g}$ com $T_{x_{0}} M$, para um ponto base escolhido $x_{0}$ e $\mathfrak{p}$ nào depende do ponto $x_{0}$ escolhido:

3. o produto interno $B$ induzido sobre $\mathfrak{p}$ pela identificação de $\mathfrak{p}$ com $\mathcal{D}_{x_{0}}$ não depende do ponto base $x_{0} \in M$ escolhido.

\section{Prova:}

$G$ é um subgrupo fechado do grupo de Lie todas as isometrias Riemannianas de $M$. portanto é um grupo de Lie. Consideremos $x_{0} \in M$ e seja $K$ o subgrupo de isotropia em $x_{0}$. Então $M$ é representado como o espaço das classes $G / K$. Agora, uma isometria sub-Riemanniana de $M$ fixa (a menos de sinais) o referencial canônico e isso implica que $K$ é finito. Como assumimos $M$ simplesmente conexa, segue que a isotropia é trivial. Portanto, a projeção $\pi: G \rightarrow M, \pi(g)=g\left(x_{0}\right)$ é um difeomorfismo, isto é, a ação de $G$ sobre $M$ é transitiva simples (em particular, $G$ é simplesmente conexo). Seja $\mathfrak{g}$ a álgebra de Lie de $G$. Temos que a aplicação induzida $\pi_{*}$ identifica $\mathfrak{g}$ com o espaço tangente $T_{x_{0}} M$. Definimos $\mathfrak{p}$ como a imagem inversa de $\mathcal{D}_{x_{0}}$ em $\mathfrak{g}$ através de $\pi_{*}$. Então $\mathfrak{g}=\mathfrak{p}+[\mathfrak{p}, \mathfrak{p}]+[[\mathfrak{p}, \mathfrak{p}], \mathfrak{p}]$, uma vez que $\mathcal{D}$ é uma distribuição de Engel. Finalmente, o subespaço $\mathfrak{p}$ de $\mathfrak{g}$ e o produto interno $g_{x_{0}}$ levantado para $\mathfrak{p}$ através de $\pi_{*}$, denotado por $B$, não dependem do $x_{0}$ escolhido, visto que $G$ age sobre $M$ preservando $\mathcal{D}$ e $g$.

Para um espaço homogêneo sub-Riemanniano $(M, \mathcal{D}, g)$ de tipo Engel temos agora uma terna $(\mathfrak{g}, \mathfrak{p}, B)$ associada, onde $\mathfrak{g}$ é uma álgebra de Lie 4-dimensional, $\mathfrak{p}$ é um subespaço 2-dimensional de $\mathfrak{g}$ tal que $\mathfrak{g}=\mathfrak{p}+[\mathfrak{p}, \mathfrak{p}]+[[\mathfrak{p}, \mathfrak{p}], \mathfrak{p}]$ e $B$ é um produto interno sobre $\mathfrak{p}$. Úma terna $(\mathfrak{g}, \mathfrak{p}, B)$ com estas propriedades é denominada álgebra de Engel. 
Corolário 2.2 Se (M.D.g) é um espaço sub-simétrico, simplesmente conexo, f-dimensional, onde $\mathcal{D}$ é uma distribuição de Engel, então sua álgebra de Engel associada (g, p, B) possui as propriedades adicionais: $[[[\mathfrak{p}, \mathfrak{p}], \mathfrak{p}], \mathfrak{p}] \subset[\mathfrak{p}, \mathfrak{p}]$ e $\operatorname{dim}(\mathfrak{p}+[[\mathfrak{p}, \mathfrak{p}], \mathfrak{p}])=3$; em particular $\mathfrak{g} e ́$ involutiva.

\section{Prova:}

Neste caso, g possui uma involução $s$ dada por $A d_{\psi}: \mathfrak{g} \rightarrow \mathfrak{g}$, onde $\iota$ é a sub-simetria em $x_{0}$. Observando então, que a $A d_{K}$-ação sobre g e a $K$-ação sobre $T_{x_{0}} \cdot K$ são $\pi_{*}$-equivalentes, temos a inclusào $\mathfrak{p} \subset \mathfrak{g}^{-}$(onde $\mathfrak{g}^{-}$denota o auto-espaço -1 de $s$ ). Assim, a decomposição de $\mathfrak{g}$ nos \pm 1 auto-espaços de $\mathfrak{s}, \mathfrak{g}=\mathfrak{g}^{+} \oplus \mathfrak{g}^{-}$, satisfaz $\mathfrak{g}^{+}=[\mathfrak{p}, \mathfrak{p}], \mathfrak{g}^{-}=\mathfrak{p}+[[\mathfrak{p}, \mathfrak{p}], \mathfrak{p}]$ e isto conclui a demonstraçào.

Reciprocamente, dada uma álgebra de Engel $(\mathfrak{g}, \mathfrak{p}, B)$ podemos construir um espaço homogêneo sub-Riemanniano de tipo Engel como segue. Seja $M=G$ o grupo de Lie simplesmente conexo com álgebra de Lie $\mathfrak{g} ; \mathcal{D}$ a distribuição $G$-invariante sobre $G$ tal que $\mathcal{D}_{e}=\mathfrak{p}$ e portanto $\mathcal{D}_{m}=\left(d L_{m}\right)_{e}\left(\mathcal{D}_{e}\right), \forall m \in G$, onde $L_{m}$ denota o automorfismo translação à esquerda por $m$ sobre $G$. Devido às hipóteses sobre $\mathfrak{p}$ e $\mathfrak{g}$ temos que $\mathcal{D}$ assim construida é uma distribuição de Engel. Seja $g$ a métrica $G$-invariante sobre $\mathcal{D}$ determinada por $B$ tal que $g_{e}=B$, isto é, $g_{m}\left(X_{m}, Y_{m}\right)=g_{e}\left(\left(d L_{m}^{-1}\right)_{m}\left(X_{m}\right),\left(d L_{m}^{-1}\right)_{m}\left(Y_{m}\right)\right), \forall m \in G$. Se a terna possui as propriedades adicionais $[[[\mathfrak{p}, \mathfrak{p}], \mathfrak{p}], \mathfrak{p}] \subset[\mathfrak{p}, \mathfrak{p}]$ e $\operatorname{dim}(\mathfrak{p}+[[\mathfrak{p}, \mathfrak{p}], \mathfrak{p}])=3$ então o automorfismo involutivo $s$ de $\mathfrak{g}$, que é +1 sobre $[\mathfrak{p}, \mathfrak{p}]$ e é -1 sobre $\mathfrak{p}+[[\mathfrak{p}, \mathfrak{p}], \mathfrak{p}]$, induz um automorfismo $\psi$ em $G$ que é uma isometria sub-Riemanniana de $G$ e $\psi_{*}=s$. Portanto $\psi$ é a sub-simetria na identidade e $L_{m}$ ○ $\psi \circ L_{m^{-1}}: G \rightarrow G$ é a sub-simetria em $m$, assim $G$ é sub-simétrico.

Observação 2.1 Pode-se provar que todo espaço sub-simétrico, simplesmente conexo, de tipo Engel, é também um espaço sub-simétrico de contato ímpar, da seguinte maneira: Dado $(M, \mathcal{D}, g)$ espaço sub-simétrico de tipo Engel, simplesmente conexo, tome seus associados $(\mathfrak{g}, \mathfrak{p}, B)$. Uma vez que $\mathfrak{g}$ possui uma estrutura involutiva, $\mathfrak{g}^{+}=\mathfrak{p}+[[\mathfrak{p}, \mathfrak{p}], \mathfrak{p}], \mathfrak{g}^{-}=[\mathfrak{p}, \mathfrak{p}]$ e $\operatorname{dim} \mathfrak{g}^{+}=3$, considere a sub-ALIO (de contato ímpar) abstrata $\left(\mathfrak{g}, \mathfrak{p}^{\prime}, B^{\prime}\right)$ (ie, $\mathfrak{g}$ é uma álgebra de Lie real 4-dimensional, $\mathfrak{p}^{\prime}$ é um subespaço 3-dimensional de $\mathfrak{g}$ tal que $\mathfrak{g}=\mathfrak{p}^{\prime}+\left[\mathfrak{p}^{\prime}, \mathfrak{p}^{\prime}\right]$, $\left[\left[\mathfrak{p}^{\prime}, \mathfrak{p}^{\prime}\right], \mathfrak{p}^{\prime}\right] \subset \mathfrak{p}^{\prime}$ e $B^{\prime}$ é um produto interno sobre $\left.\mathfrak{p}\right)$, onde $\mathfrak{p}^{\prime}=\mathfrak{g}^{+}$e $B^{\prime}$ é a restrição à $\mathfrak{p}^{\prime}$ da métrica canônica definida em $\mathfrak{g}$ via a aplicação induzida $\pi_{*}$. $\dot{A}$ terna $\left(\mathfrak{g}, \mathfrak{p}^{\prime}, B^{\prime}\right)$ corresponde um espaço sub-simétrico de contato ímpar $\left(G \equiv M, \mathcal{D}^{\prime}, g^{\prime}\right)$, onde $G^{\prime}$ é o grupo simplesmente conexo com álgebra de Lie $\mathfrak{g} ; \mathcal{D}^{\prime}$ é a distribuição $G$-invariante definida em $G$, tal que $\mathcal{D}_{e}^{\prime}=\mathfrak{p}^{\prime}$ e $\mathfrak{g}^{\prime}$ é a métrica $G$-invariante definida em $\mathcal{D}^{\prime}$, tal que $g_{e}^{\prime}=B^{\prime}$. Maiores detalhes sobre esta última afirmação podem ser encontrados em [3]. 


\subsection{A classificação dos espaços sub-simétricos com dis- tribuição de Engel}

Vesta seção apresentaremos a classificação dos espaços sub-simétricos com distribuição de Engel. Mais precisamente, mostraremos que existem exatamente 10 classes de exemplos destes espaços, são elas o recobrimento universal (denotados com um til) do: produto direto do grupo de movimentos próprios do plano Euclidiano $E u c_{2}^{+}=S O(2) \bowtie \mathbb{R}^{2}$ com $\mathbb{R}$; produto direto do grupo próprio ortocronológico de Poincaré Poinc $c_{2}^{+}=S O_{e}(1.1) \bowtie \mathbb{R}^{2}$ com $\mathbb{R}$; grupo unitário indefinido $U(1,1)$ (este espaço admite quatro classes distintas de estruturas sub-Riemannianas $(\mathcal{D}, g)$ nào equivalentes - isto segue olhando-se os invariantes na tabela 2.1 - distinguimos estes espaços um do outro por "linhas"); grupo de Engel $E^{4}$ cuja álgebra de Lie $e_{4}$ possui uma base $\beta=\left\{x_{1}, x_{2}, y=\left[x_{1}, x_{2}\right], z=\left[x_{2}, y\right]\right\}$ satisfazendo $\left[y, x_{1}\right]=\left[z, x_{1}\right]=$ $[y . z]=\left[z, x_{2}\right]=0$; grupo unitário $U(2)$; produto semi-direto do grupo ortogonal especial $\varsigma O(2)$ com o grupo de Heisenberg $H^{3}$; produto semi-direto do grupo ortogonal indefinido $5 \mathrm{O}_{e}(1,1)$ com o grupo de Heisenberg $H^{3}$.

Observação 2.2 A lista detalhada destes espaços com suas respectivas distribuições, métricas e invariantes se encontra no final deste capítulo na tabela 2.1.

Observação 2.3 Embora o teorema (2.1) e sua recíproca permitam lidar com o problema de classificação dos espaços homogêneos sub-Riemannianos em geral. uma análise preliminar mostra que os exemplos geometricamente mais significativos pertencem a classe dos espaços sub-simétricos.

Retomamos agora o problema de classificação. Seja $(G, \mathcal{D}, g)$ um espaço sub-simétrico simplesmente conexo de dimensão $4 \mathrm{com}$ uma distribuição de Engel. Considerem-se seus associados $(\mathfrak{g}, \mathfrak{p}, B)$. Entào, $\operatorname{dim} \mathfrak{g}=4, \operatorname{dim} \mathfrak{p}=2, \operatorname{dim}[\mathfrak{p}, \mathfrak{p}]=1, \operatorname{dim}(\mathfrak{p}+[[\mathfrak{p}, \mathfrak{p}], \mathfrak{p}])=3$, $\mathfrak{g}=\mathfrak{p}+[\mathfrak{p}, \mathfrak{p}]+[[\mathfrak{p}, \mathfrak{p}], \mathfrak{p}], \quad[[[\mathfrak{p}, \mathfrak{p}], \mathfrak{p}], \mathfrak{p}] \subset[\mathfrak{p}, \mathfrak{p}]$ e $B$ é um produto interno sobre $\mathfrak{p}$. Sob estas hipóteses, utilizando as identidades de Jacobi, temos que a base ortonormal de $\mathfrak{g}$, $3=\left\{Y_{1}, Y_{2}, \xi_{3}, \xi_{4}\right\}$, dada pelo referencial canônico, satisfaz: 


$$
\begin{aligned}
& {\left[Y_{1}, Y_{2}\right]=\xi_{3}} \\
& {\left[Y_{1}, \xi_{3}\right]=\xi_{4}} \\
& {\left[Y_{1}, \xi_{4}\right]=-a \xi_{3}} \\
& {\left[Y_{2}, \xi_{3}\right]=-b Y_{1}-c Y_{2}} \\
& {\left[Y_{2}, \xi_{4}\right]=-c \xi_{3}} \\
& {\left[\xi_{3}, \xi_{4}\right]=-a\left(b Y_{1}+c Y_{2}\right)-c \xi_{4}}
\end{aligned}
$$

Portanto. neste caso, as equações de estrutura associadas a conexão adaptada (definida no capítulo 1), expressam-se no co-referencial $\left\{\theta^{1}, \theta^{2}, \theta^{3}, \theta^{4}\right\}$ dual do referencial canônico $\left\{Y_{1}, Y_{2}, \xi_{3}, \xi_{4}\right\}$, como:

$$
\begin{aligned}
& d \theta^{1}=\theta^{2} \wedge \frac{1}{2} b \theta^{3}+\theta^{3} \wedge\left(-\frac{1}{2} b \theta^{2}\right)+a b \theta^{3} \wedge \theta^{4} \\
& d \theta^{2}=\theta^{1} \wedge\left(-\frac{1}{2} b \theta^{3}\right)+\theta^{3} \wedge\left(-\frac{1}{2} b \theta^{1}-c \theta^{2}\right)+a c \theta^{3} \wedge \theta^{4} \\
& d \theta^{3}=\theta^{4} \wedge\left(-\frac{1}{2}(1+a) \theta^{1}-\frac{1}{2} c \theta^{2}-c \theta^{4}\right)+\frac{1}{2}(1-a) \theta^{4} \wedge \theta^{1}-\frac{1}{2} c \theta^{4} \wedge \theta^{2}-\theta^{1} \wedge \theta^{2} \\
& d \theta^{4}=\theta^{3} \wedge\left(\frac{1}{2}(1+a) \theta^{1}+\frac{1}{2} c \theta^{2}+c \theta^{4}\right)+\frac{1}{2}(1-a) \theta^{3} \wedge \theta^{1}-\left(\frac{1}{2} c \theta^{3}\right) \wedge \theta^{2}
\end{aligned}
$$

de onde:

$$
\begin{aligned}
\omega_{1}^{2} & =-\frac{1}{2} b \theta^{3} \\
\omega_{3}^{4} & =\frac{1}{2}(1+a) \theta^{1}+\frac{1}{2} c \theta^{2}+c \theta^{4} \\
\tau_{3}^{1} & =-\frac{1}{2} b \theta^{2} \\
\tau_{3}^{2} & =-\frac{1}{2} b \theta^{1}-c \theta^{2} \\
\tau_{4}^{1} & =0
\end{aligned}
$$




$$
\begin{aligned}
& \tau_{4}^{2}=0 \\
& \sigma_{1}^{3}=\frac{1}{2}(1-a) \theta^{4} \\
& \sigma_{2}^{3}=-\frac{1}{2} c \theta^{4} \\
& \sigma_{1}^{4}=\frac{1}{2}(1-a) \theta^{3} \\
& \sigma_{2}^{4}=-\frac{1}{2} c \theta^{3}
\end{aligned}
$$

As curvaturas tangenciais e normais são dadas por:

$$
\begin{aligned}
& \Omega=d \omega_{1}^{2}\left(Y_{1}, Y_{2}\right)=\frac{1}{2}<\left[\xi_{3}, Y_{2}\right], Y_{1}>=\frac{1}{2} b \\
& \mu=d \omega_{3}^{4}\left(\xi_{3}, \xi_{4}\right)=\frac{1}{2}\left\{(1+a)\left(a b+c^{2}\right)+c^{2}\right\}
\end{aligned}
$$

Além das curvaturas calculamos os invariantes relacionados com as sub-torçòes. $\tau$ e $\sigma$. definidos no capítulo 1, na seção 1.3, e obtivemos:

$$
\begin{aligned}
\hat{\sigma}_{1}=\hat{\sigma}_{2} & =\frac{1}{4}\left\{(1-a)^{2}+c^{2}\right\} \\
\text { traço de } \hat{\tau} & =\frac{1}{2} b^{2}+c^{2} \\
\text { determinante de } \hat{\tau} & =\frac{1}{16} b^{4}
\end{aligned}
$$

A existência do referencial canônico definido sobre a variedade nos permite definir. além dos invariantes citados acima, outros invariantes geométricos. Tendo em vista o objetivo de classificação definimos os invariantes que seguem:

$$
\begin{aligned}
& \mu_{1}=<\left[\xi_{4}, Y_{1}\right], \xi_{3}>=a \\
& \mu_{2}=<\left[\xi_{4}, Y_{1}\right], \xi_{3}>.<\left[\xi_{3}, Y_{2}\right], Y_{1}>+\left(<\left[\xi_{4}, Y_{2}\right], \xi_{3}>\right)^{2}=a b+c^{2}
\end{aligned}
$$

Salientamos que $2 \Omega, \mu_{1}$ e $\mu_{2}$ independem das orientações escolhidas sobre $\mathcal{D}$ e $M$. 
Temos dois casos a considerar:

Primeiro caso: $\quad \operatorname{dim}[[\mathfrak{p}, \mathfrak{p}], \mathfrak{p}]=1$

Neste caso, $b=c=0$, e então $\beta=\left\{Y_{1}, Y_{2}, \xi_{3}, \xi_{4}\right\}$ é uma base ortonormal de gat, satisfazendo:

$$
\begin{aligned}
& {\left[Y_{1}, Y_{2}\right]=\xi_{3}} \\
& {\left[Y_{1}, \xi_{3}\right]=\xi_{4}} \\
& {\left[Y_{1}, \xi_{4}\right]=-a \xi_{3}} \\
& {\left[Y_{2}, \xi_{3}\right]=0} \\
& {\left[Y_{2}, \xi_{4}\right]=0} \\
& {\left[\xi_{3}, \xi_{4}\right]=0}
\end{aligned}
$$

Se $a=0, \mathfrak{g}$ é a álgebra de Lie do grupo de Engel $E^{4}$.

Se $a \neq 0$, a transformação

$$
\begin{aligned}
Y_{1}^{\prime} & =\frac{1}{\sqrt{|a|}} Y_{1} \\
Y_{2}^{\prime} & =\xi_{3} \\
\xi_{3}^{\prime} & =\frac{1}{\sqrt{|a|}} \xi_{4} \\
\xi_{4}^{\prime} & =a Y_{2}+\xi_{4}
\end{aligned}
$$

é tal que

$$
\begin{aligned}
& {\left[Y_{1}^{\prime}, Y_{2}^{\prime}\right]=\xi_{3}^{\prime}} \\
& {\left[Y_{1}^{\prime}, \xi_{3}^{\prime}\right]=-\operatorname{sgn}(a) Y_{2}^{\prime \prime}}
\end{aligned}
$$




$$
\begin{aligned}
& {\left[Y_{1}^{\prime \prime}, \xi_{4}^{\prime}\right]=0} \\
& {\left[Y_{2}^{\prime \prime}, \xi_{3}^{\prime}\right]=0} \\
& {\left[Y_{2}^{\prime}, \xi_{4}^{\prime}\right]=0} \\
& {\left[\xi_{3}^{\prime}, \xi_{4}^{\prime}\right]=0}
\end{aligned}
$$

portanto, é um isomorfismo sobre a álgebra de Lie de

$$
\begin{cases}\widetilde{\text { Uuc }_{2}^{+}} \times \mathbb{R} & \text { se } a>0 \\ \text { Poinc }_{2}^{+} \times \mathbb{R} & \text { se } a<0\end{cases}
$$

Segundo caso: $\quad \operatorname{dim}[[\mathfrak{p}, \mathfrak{p}], \mathfrak{p}]=2$

Temos duas possibilidades a considerar:

Possibilidade A): $\quad\left[Y_{2}, \xi_{3}\right]$ pertence ao núcleo da $\mathcal{L}$

Neste caso, $b=0$ e $c \neq 0$, e então $\beta=\left\{Y_{1}, Y_{2}, \xi_{3}, \xi_{4}\right\}$ é uma base ortonormal de $\mathfrak{g}$, satisfazendo:

$$
\begin{aligned}
& {\left[Y_{1}, Y_{2}\right]=\xi_{3}} \\
& {\left[Y_{1}, \xi_{3}\right]=\xi_{4}} \\
& {\left[Y_{1}, \xi_{4}\right]=-a \xi_{3}} \\
& {\left[Y_{2}, \xi_{3}\right]=-c Y_{2}} \\
& {\left[Y_{2}, \xi_{4}\right]=-c \xi_{3}} \\
& {\left[\xi_{3}, \xi_{4}\right]=-a c Y_{2}-c \xi_{4}}
\end{aligned}
$$

A transformação

$$
Y_{1}^{\prime}=\frac{1}{c} \xi_{3}
$$




$$
\begin{aligned}
Y_{2}^{\prime} & =\frac{1}{c \sqrt{2}}\left(\left(1-\frac{a}{2}\right) Y_{2}-\xi_{4}\right) \\
\xi_{3}^{\prime} & =\frac{1}{c \sqrt{2}}\left(\left(1+\frac{a}{2}\right) Y_{2}+\xi_{4}\right) \\
\xi_{4}^{\prime} & =Y_{1}-\frac{a}{c} Y_{2}-\frac{1}{c} \xi_{4}
\end{aligned}
$$

é tal que

$$
\begin{aligned}
& {\left[Y_{1}^{\prime}, Y_{2}^{\prime}\right]=\xi_{3}^{\prime}} \\
& {\left[Y_{1}^{\prime}, \xi_{3}^{\prime}\right]=Y_{2}^{\prime}} \\
& {\left[Y_{1}^{\prime}, \xi_{4}^{\prime}\right]=0} \\
& {\left[Y_{2}^{\prime}, \xi_{3}^{\prime}\right]=-Y_{1}^{\prime}} \\
& {\left[Y_{2}^{\prime}, \xi_{4}^{\prime}\right]=0} \\
& {\left[\xi_{3}^{\prime}, \xi_{4}^{\prime}\right]=0}
\end{aligned}
$$

portanto, é um isomorfismo sobre a álgebra de Lie de $\widetilde{U(1,1)}$.

Possibilidade $\mathrm{B}): \quad\left[Y_{2}, \xi_{3}\right] \quad$ não pertence ao núcleo da $\mathcal{L}$

Neste caso, $b \neq 0$, e então $\beta=\left\{Y_{1}, Y_{2}, \xi_{3}, \xi_{4}\right\}$ é uma base ortonormal de $\mathfrak{g}$, satisfazendo:

$$
\begin{aligned}
& {\left[Y_{1}, Y_{2}\right]=\xi_{3}} \\
& {\left[Y_{1}, \xi_{3}\right]=\xi_{4}} \\
& {\left[Y_{1}, \xi_{4}\right]=-a \xi_{3}} \\
& {\left[Y_{2}, \xi_{3}\right]=-b Y_{1}-c Y_{2}} \\
& {\left[Y_{2}, \xi_{4}\right]=-c \xi_{3}} \\
& {\left[\xi_{3}, \xi_{4}\right]=-a\left(b Y_{1}+c Y_{2}\right)-c \xi_{4}} \\
& (a \in \mathbb{R}, b \neq 0, c \geq 0)
\end{aligned}
$$


Se $\left(a b+c^{2}\right)=0$, a transformaçào

$$
\begin{aligned}
Y_{1}^{\prime} & =-\frac{1}{\sqrt{|b|}} Y_{2} \\
Y_{2}^{\prime} & =b Y_{1}+c Y_{2} \\
\xi_{3}^{\prime} & =\frac{1}{\sqrt{|b|}} b \xi_{3} \\
\xi_{4}^{\prime} & =-\frac{1}{\sqrt{|b|}} b^{2} \xi_{4}+\frac{1}{\sqrt{|b|}} b c\left(b Y_{1}+c Y_{2}\right)
\end{aligned}
$$

é tal que

$$
\begin{aligned}
& {\left[Y_{1}^{\prime}, Y_{2}^{\prime}\right]=\xi_{3}^{\prime}} \\
& {\left[Y_{1}^{\prime}, \xi_{3}^{\prime}\right]=\operatorname{sgn}(b) Y_{2}^{\prime}} \\
& {\left[Y_{1}^{\prime}, \xi_{4}^{\prime}\right]=0} \\
& {\left[Y_{2}^{\prime}, \xi_{3}^{\prime}\right]=-\xi_{4}^{\prime}} \\
& {\left[Y_{2}^{\prime}, \xi_{4}^{\prime}\right]=0} \\
& {\left[\xi_{3}^{\prime}, \xi_{4}^{\prime}\right]=0}
\end{aligned}
$$

portanto, é um isomorfismo sobre a álgebra de Lie de

$$
\begin{cases}S O \widetilde{(2)} H^{3} & \text { se } b<0 ; \\ S O_{e}(1,1) \bowtie H^{3} & \text { se } b>0 .\end{cases}
$$

Se $\left(a b+c^{2}\right) \neq 0$, a transformação

$$
Y_{1}^{\prime}=-\frac{1}{b\left(a b+c^{2}\right) \sqrt{|b|}}\left\{b^{2} \xi_{4}-b c\left(b Y_{1}+c Y_{2}\right)\right\}
$$




$$
\begin{aligned}
Y_{2}^{\prime} & =\frac{1}{\sqrt{\left|b\left(a b+c^{2}\right)\right|}}\left(b Y_{1}+c Y_{2}\right) \\
\xi_{3}^{\prime} & =-\frac{1}{|b| \sqrt{\left|a b+c^{2}\right|}}\left(b \xi_{3}\right) \\
\xi_{4}^{\prime} & =a b^{2} Y_{2}+b^{2} \xi_{4}-b^{2} c Y_{1}
\end{aligned}
$$

é tal que

$$
\begin{aligned}
& {\left[Y_{1}^{\prime}, Y_{2}^{\prime}\right]=\xi_{3}^{\prime}} \\
& {\left[Y_{1}^{\prime}, \xi_{3}^{\prime}\right]=\operatorname{sgn}(b) Y_{2}^{\prime}} \\
& {\left[Y_{1}^{\prime}, \xi_{4}^{\prime}\right]=0} \\
& {\left[Y_{2}^{\prime}, \xi_{3}^{\prime}\right]=\operatorname{sgn}\left[b \cdot\left(a b+c^{2}\right)\right] Y_{1}^{\prime}} \\
& {\left[Y_{2}^{\prime}, \xi_{4}^{\prime}\right]=0} \\
& {\left[\xi_{3}^{\prime}, \xi_{4}^{\prime}\right]=0}
\end{aligned}
$$

portanto, é um isomorfismo sobre a álgebra de Lie de

$$
\left\{\begin{array}{lll}
\widetilde{U(1,1)^{\prime}} & \text { se } b>0, & \left(a b+c^{2}\right)<0 \\
\widetilde{U(1,1)^{\prime \prime}} & \text { se } b<0, & \left(a b+c^{2}\right)>0 \\
\widetilde{U(1,1)^{\prime \prime \prime}} & \text { se } b>0, & \left(a b+c^{2}\right)>0 \\
\widetilde{U(2)} & \text { se } b<0, & \left(a b+c^{2}\right)<0 .
\end{array}\right.
$$

A classificação completa está sumarizada na tabela 2.1. Na primeira coluna, listamos representativos $G$ para os grupos de Lie 4-dimensionais simplesmente conexos, os quais são sub-simétricos de tipo Engel. Na segunda coluna, descrevemos a estrutura da álgebra de Lie g: existe uma base $\left\{Y_{1}^{\prime}, Y_{2}^{\prime \prime}, \xi_{3}^{\prime}, \xi_{4}^{\prime}\right\}$ da álgebra de Lie de $G_{r}$ tal que $\xi_{4}^{\prime}$ é central, $\xi_{3}^{\prime}=\left[Y_{1}^{\prime}, Y_{2}^{\prime}\right]$ e $A^{\prime}$ é a matriz transposta de $\operatorname{ad}_{\xi_{3}^{\prime}}$, restrita à base $\left\{Y_{1}^{\prime}, Y_{2}^{\prime}, \xi_{4}^{\prime}\right\}$. Na terceira coluna, damos uma base ortonormal $\left\{Y_{1}, Y_{2}\right\}$ de $\mathfrak{p}$. As colunas seguintes listam os invariantes: $2 \Omega, \mu_{1}$ e $\mu_{2}$. 


\begin{tabular}{|c|c|c|c|c|c|}
\hline$G$ & $A^{\prime}$ & $\left\{Y_{1}, Y_{2}\right\}$ é uma base o.n. de $\mathfrak{p}$ & $2 \Omega$ & $\mu_{1}$ & $\mu_{2}$ \\
\hline$E^{4}$ & $\left(\begin{array}{ccc}0 & 0 & -1 \\
0 & 0 & 0 \\
0 & 0 & 0\end{array}\right)$ & $\begin{array}{l}Y_{1}=Y_{1}^{\prime} \\
Y_{2}=Y_{2}^{\prime \prime}\end{array}$ & 0 & 0 & 0 \\
\hline$E u c_{2}^{+} \times \mathbb{R}$ & $\left(\begin{array}{lll}0 & 1 & 0 \\
0 & 0 & 0 \\
0 & 0 & 0\end{array}\right)$ & $\begin{array}{l}Y_{1}=\sqrt{b} Y_{1}^{\prime} \\
Y_{2}=\frac{1}{b} \xi_{4}^{\prime}-\frac{1}{\sqrt{b}} \xi_{3}^{\prime}\end{array}$ & 0 & $b$ & 0 \\
\hline Poinc $_{2}^{+} \times \mathbb{R}$ & $\left(\begin{array}{ccc}0 & -1 & 0 \\
0 & 0 & 0 \\
0 & 0 & 0\end{array}\right)$ & $\begin{array}{l}Y_{1}=\sqrt{b} Y_{1}^{\prime} \\
Y_{2}=-\frac{1}{b} \xi_{4}^{\prime}+\frac{1}{\sqrt{b}} \xi_{3}^{\prime}\end{array}$ & 0 & $-b$ & 0 \\
\hline$U(1,1)$ & $\left(\begin{array}{ccc}0 & -1 & 0 \\
1 & 0 & 0 \\
0 & 0 & 0\end{array}\right)$ & $\begin{aligned} Y_{1}= & \frac{1}{\sqrt{2}}\left(-1+\frac{a}{2}\right) Y_{2}^{\prime}+\xi_{4}^{\prime} \\
& +\frac{1}{\sqrt{2}}\left(1+\frac{a}{2}\right) \xi_{3}^{\prime} \\
Y_{2}= & \frac{c}{\sqrt{2}}\left(Y_{2}^{\prime}+\xi_{3}^{\prime}\right)\end{aligned}$ & 0 & $a$ & $c^{2}$ \\
\hline$S O \widetilde{(2) \bowtie H^{3}}$ & $\left(\begin{array}{lll}0 & 1 & 0 \\
0 & 0 & 1 \\
0 & 0 & 0\end{array}\right)$ & $\begin{array}{l}Y_{1}=-\frac{1}{b}\left(Y_{2}^{\prime}+c \sqrt{b} Y_{1}^{\prime}\right) \\
Y_{2}=-\sqrt{b} Y_{1}^{\prime}\end{array}$ & $-b$ & $a$ & 0 \\
\hline$S O_{e}(1,1) \bowtie H^{3}$ & $\left(\begin{array}{ccc}0 & -1 & 0 \\
0 & 0 & 1 \\
0 & 0 & 0\end{array}\right)$ & $\begin{array}{l}Y_{1}=\frac{1}{b}\left(Y_{2}^{\prime}+c \sqrt{b} Y_{1}^{\prime}\right) \\
Y_{2}=-\sqrt{b} Y_{1}^{\prime}\end{array}$ & $b$ & $a$ & 0 \\
\hline$\widetilde{U(2)}$ & $\left(\begin{array}{ccc}0 & 1 & 0 \\
-1 & 0 & 0 \\
0 & 0 & 0\end{array}\right)$ & $\begin{aligned} Y_{1}= & -c \sqrt{b} Y_{1}^{\prime}-\left\{\sqrt{b\left(a b+c^{2}\right)} / b\right\} Y_{2}^{\prime} \\
& -\left\{c / b\left(a b+c^{2}\right)\right\} \xi_{4}^{\prime} \\
Y_{2}= & \sqrt{b} Y_{1}^{\prime}+\left\{1 / b\left(a b+c^{2}\right)\right\} \xi_{4}^{\prime}\end{aligned}$ & $-b$ & $a$ & $-\left(a b+c^{2}\right)$ \\
\hline$\widehat{U(1,1)^{\prime}}$ & $\left(\begin{array}{ccc}0 & -1 & 0 \\
1 & 0 & 0 \\
0 & 0 & 0\end{array}\right)$ & $\begin{aligned} Y_{1}= & -c \sqrt{b} Y_{1}^{\prime}+\left\{\sqrt{\left.b\left(a b+c^{2}\right) / b\right\} Y_{2}^{\prime}}\right. \\
& +\left\{c / b\left(a b+c^{2}\right)\right\} \xi_{4}^{\prime} \\
Y_{2}= & \sqrt{b} Y_{1}^{\prime}-\left\{1 / b\left(a b+c^{2}\right)\right\} \xi_{4}^{\prime}\end{aligned}$ & $b$ & $a$ & $-\left(a b+c^{2}\right)$ \\
\hline$\widetilde{U(1,1)^{\prime \prime}}$ & $\left(\begin{array}{ccc}0 & -1 & 0 \\
1 & 0 & 0 \\
0 & 0 & 0\end{array}\right)$ & $\begin{aligned} Y_{1}= & -c \sqrt{b} \xi_{3}^{\prime}-\left\{\sqrt{b\left(a b+c^{2}\right)} / b\right\} Y_{2}^{\prime} \\
& +\left\{c / b\left(a b+c^{2}\right)\right\} \xi_{4}^{\prime} \\
Y_{2}= & \sqrt{b} \xi_{3}^{\prime}-\left\{1 / b\left(a b+c^{2}\right)\right\} \xi_{4}^{\prime}\end{aligned}$ & $-b$ & $a$ & $a b+c^{2}$ \\
\hline$\widetilde{U(1,1)^{\prime \prime \prime}}$ & $\left(\begin{array}{ccc}0 & -1 & 0 \\
1 & 0 & 0 \\
0 & 0 & 0\end{array}\right)$ & $\begin{aligned} Y_{1}= & -c \sqrt{b} Y_{1}^{\prime}+\left\{\sqrt{b\left(a b+c^{2}\right)} / b\right\} \xi_{3}^{\prime} \\
& -\left\{c / b\left(a b+c^{2}\right)\right\} \xi_{4}^{\prime} \\
Y_{2}= & \sqrt{b} Y_{1}^{\prime}+\left\{1 / b\left(a b+c^{2}\right)\right\} \xi_{4}^{\prime}\end{aligned}$ & $b$ & $a$ & $a b+c^{2}$ \\
\hline
\end{tabular}

Tabela 2.1: Espaços sub-simétricos de tipo Engel: $\left\{Y_{1}^{\prime}, Y_{2}^{\prime}, \xi_{3}^{\prime}=\left[Y_{1}^{\prime}, Y_{2}^{\prime}\right], \xi_{4}^{\prime}\right\}$ é uma base da álgebra de Lie de $G$, tal que $\xi_{4}^{\prime}$ é central; $A^{\prime}$ é a matriz transposta de $\operatorname{ad}_{\xi_{3}^{\prime}}$ restrita à base $\left\{Y_{1}^{\prime}, Y_{2}^{\prime}, \xi_{4}^{\prime}\right\}: 2 \Omega, \mu_{1}$ e $\mu_{2}$ são invariantes; $a, b, c$ são parâmetros com $a \in \mathbb{R}, b>0$, e $c>0$ é tal que $\left(a b+c^{2}\right)>0$. 


\section{Capítulo 3}

\section{Estrutura dos espaços sub-simétricos com distribuição fortemente geradora por colchetes}

Neste capítulo definiremos distribuição fortemente geradora por colchetes. Construiremos uma métrica Riemanniana canônica para uma variedade sub-Riemanniana cuja distribuição associada é deste tipo. Apresentaremos um teorema de estrutura para espaços sub-simétricos munidos com este tipo de distribuição, via álgebras de Lie involutivas sub-ortogonais e utilizaremos esta estrutura para caracterizar as sub-torções.

\subsection{Distribuição fortemente geradora por colchetes}

Fixada uma distribuição $\mathcal{D}$ em uma variedade diferenciável $M$, dizemos que a distribuição $\mathcal{D}$ é fortemente geradora por colchetes quando dado $x \in . M$ e qualquer seção local $Z$ de $\mathcal{D}$ $\operatorname{com} Z(x) \neq 0$, obtemos:

$$
T_{x} M=\mathcal{D}_{x}+\left\{\left[Z, X^{\prime}\right]_{x}: X \text { seçào de } \mathcal{D}\right\}
$$

No que segue, $\mathcal{L}$ denota a forma de Levi de $\mathcal{D}$ definida por:

$$
\mathcal{L}_{x}: \mathcal{D}_{x} \times \mathcal{D}_{x} \rightarrow T_{x} M / \mathcal{D}_{x}, \quad \mathcal{L}_{x}(\mathrm{v}, \mathrm{w})=[V, W]_{x} \quad \bmod \mathcal{D}_{x}
$$

onde $V, W$ são extensões de $\mathrm{v}, \mathrm{w}$ às seções de $\mathcal{D}$.

Observamos que, em termos da forma de Levi de $\mathcal{D}$. a condição $\mathcal{D}$ fortemente geradora por colchetes é equivalente a: dado $x \in M$ e $\mathrm{v} \in \mathcal{D}_{x}, \forall \neq 0$, o operador linear $\mathcal{L}_{x}(\mathrm{v}, \cdot)$ é sobrejetor. É claro que quando $\mathcal{D}$ tem codimensào um essa condição é equivalente à condição de contato. 
Dada, uma variedade diferenciável $M$, seja $T^{*} . M=\left\{(x, \lambda): x \in M \cdot \lambda \in T_{x} M^{*}\right\}$ o fibrado cotangente de $M$. Denotemos por $\mathcal{D}^{0}$ o subfibrado vetorial de $T^{*} M$ cuja fibra sobre o ponto $x \in M$ é o anulador de $\mathcal{D}_{x}$ em $T_{x} . M$ ie, $\mathcal{D}^{0}=\left\{(x, \lambda) \in T^{*} M / \lambda\left(\mathcal{D}_{x}\right)=0\right\}$, por simplicidade diremos apenas que $\mathcal{D}^{0} \subset T^{*} . Y$ é o anulador de $\mathcal{D}$ em $T . M$.

\subsection{Métrica associada a uma variedade sub-Rieman- niana com distribuição fortemente geradora por colchetes}

Os resultados desta seçào correspondem a uma interpretação minuciosa de ([13], seção 7$)$. Seja $(M, \mathcal{D}, g)$ uma variedade sub-Riemanniana, $\mathcal{D}$ distribuição fortemente geradora por colchetes de posto n e codimensão d. Sejam $x \in M, \omega_{x} \in \mathcal{D}_{x}^{0}$ e $\omega$ uma seção local de $\mathcal{D}^{0}$ passando por $\omega_{x}$. Se $X . Y$ sào seçòes locais de $\mathcal{D}$ próximas de $x$, então:

$$
d \omega(X, Y)=X(\omega(Y))-Y(\omega(X))-\omega\left(\left[X, Y^{\prime}\right]\right)=-\omega([X, Y]),
$$

em particular, $d \omega\left(X_{x}, Y_{x}\right)=-\omega_{x}\left([X, Y]_{x}\right)$, portanto $\left.d \omega\right|_{\mathcal{D}_{x} \times \mathcal{D}_{x}}$ depende somente de $\omega_{x}$ e temos então bem definida a aplicação linear:

$$
\begin{aligned}
& J_{x}: \mathcal{D}_{x}^{0} \longrightarrow \mathcal{D}_{x}^{*} \wedge \mathcal{D}_{x}^{*} \\
& \left.\omega_{x} \longmapsto d \omega\right|_{\mathcal{D}_{x} \times \mathcal{D}_{x}}: \mathcal{D}_{x} \times \mathcal{D}_{x} \longrightarrow \mathbb{I R} \\
& \left(X_{x}, Y_{x}\right) \longmapsto d \omega\left(X_{x}, Y_{x}\right)=-\omega_{x}\left([X, Y]_{x}\right)
\end{aligned}
$$

onde $X, Y$ são extensòes horizontais de $X_{x}, Y_{x}$.

Observe que a aplicação $J_{x}$ é injetora, pois se $\omega_{x} \in \mathcal{D}_{x}^{0}$ (ie $\left.\omega_{x}\left(\mathcal{D}_{x}\right)=0\right)$ e $\omega_{x}\left([X, Y]_{x}\right)=0$ $\forall X, Y$ seçòes locais de $\mathcal{D}$ próximas de $x$, então $\omega_{x}\left(T_{x} M\right)=0$, ou seja $\omega_{x}$ é identicamente nula, uma vez que $\mathcal{D}$ é fortemente geradora por colchetes.

Assim sendo, como a métrica $g$ definida em $\mathcal{D}$ induz naturalmente uma métrica Riemanniana em $\mathcal{D}^{*} \wedge \mathcal{D}^{*}$, temos uma métrica Riemanniana natural, denotada por $g^{0}$, definida em $\mathcal{D}^{0}$ induzida de $\mathcal{D}^{*} \wedge \mathcal{D}^{*}$. via $J\left(J: \mathcal{D}^{0} \rightarrow \mathcal{D}^{*} \wedge \mathcal{D}^{*}\right)$.

Para cada seção local $\omega \in \mathcal{D}^{0}$ passando pelo ponto $x \in M$, definimos a aplicação linear:

$$
\begin{aligned}
& (J \omega)_{x}: \mathcal{D}_{x} \longrightarrow T_{x} M^{*} \\
& v \longmapsto i_{v} \circ d \omega: T_{x} M \longrightarrow \mathbb{R} \\
& u \longmapsto d \omega(v, u)
\end{aligned}
$$

Se $\omega_{x} \neq 0$ então $\left(J_{\omega}\right)_{x}$ é injetora. De fato, $(J \omega)_{x}(v)=0$ é equivalente a $d \omega\left(v, T_{x} M\right)=0$, em particular $d \omega\left(v, \mathcal{D}_{x}\right)=0$, ou seja $\omega_{x}\left([V, \mathcal{D}]_{x}\right)=0, \forall V$ extensão horizontal de $v$ e isto implica que $v=0$ ou $\omega_{x}\left(T_{r}: I I\right)=0$ (visto que, da condição fortemente geradora por colchetes, 
se $r \neq 0$, tem-se $T_{x} M=\mathcal{D}_{x}+[V \mathcal{D}]_{x}$, e também por hipótese $\left.\omega_{x}\left(\mathcal{D}_{x}\right)=0\right)$. Agora. por hipótese $\omega_{x} \neq 0$, assim a única possibilidade é $v=0$, ou seja $(J \omega)_{x}$ é injetora.

Da injetividade de $(J \omega)_{x}$ segue que $\operatorname{dim}(J \omega)_{x}\left(\mathcal{D}_{x}\right)=\operatorname{dim} \mathcal{D}_{x}$. Também se $\omega_{x} \neq 0$ temos que $\mathcal{D}_{x}^{0} \cap(J \omega)_{x}\left(\mathcal{D}_{x}\right)=0$, pois se $i_{v} \circ d \omega \in \mathcal{D}_{x}^{0}$ para algum $v \in \mathcal{D}_{x}$ então $\left(i_{v} \circ d \omega\right)\left(\mathcal{D}_{x}\right)=$ $d_{\omega}\left(v, \mathcal{D}_{x}\right)=0$, portanto $v=0$ (pelo mesmo argumento acima, já que $\dot{\omega}_{x} \neq 0$ ) e assim $i_{2} \circ d \omega=0$. Observando-se então que $\mathcal{D}_{x}^{0}+(J \omega)_{x}\left(\mathcal{D}_{x}\right) \subset T_{x} M^{*}$. segue que:

$$
T_{x} M^{*}=\mathcal{D}_{x}^{0} \oplus(J \omega)_{x}\left(\mathcal{D}_{x}\right) \quad \forall \text { seçào local } \omega \in \mathcal{D}^{0} \operatorname{com} \omega_{x} \neq 0 .
$$

Proposição 3.1 Para cada $x \in M$ e para cada seção local $\omega \in \mathcal{D}^{0}$. passando pelo ponto $x$ com $\omega_{x} \neq 0$, existe um único vetor $\left(\xi_{\omega}\right)_{x} \in\left(T_{x} M-\mathcal{D}_{x}\right)$ tal que :

i) $\omega_{x}\left(\xi_{\omega}\right)_{x}=1$;

ii) $\eta_{x}\left(\xi_{\omega}\right)_{x}=0 \quad \forall \eta_{x} \perp \omega_{x} \quad\left(\eta_{x} \in \mathcal{D}_{x}^{0}\right)$;

iii) $\left.\left(i_{\left(\xi_{\omega_{x}}\right)} \circ d \omega\right)\right|_{\mathcal{D}_{x}}=0$.

\section{Prova:}

Consideremos o $(n+d-1)$ subespaço de $T_{x} M^{*}$, dado por $\left(S_{\omega}\right)_{x}=\left\{\eta_{x} \in \mathcal{D}_{x}^{0}: \eta_{x} \perp \omega_{x}\right\} \oplus$ $(J \omega)_{x}\left(\mathcal{D}_{x}\right)$. Notemos que $\left(S_{\omega}\right)_{x}$ é transversal ao subespaço $\mathbb{R} \omega_{x}$. Seja agora, $\left(L_{\omega}\right)_{x}$ o subespaço 1-dimensional de $T_{x} M$ anulado por $\left(S_{\omega}\right)_{x}$. Então, temos que $\left(L_{\omega}\right)_{x}$ é transversal ao núcleo de $\omega_{x}$. Definimos $\left(\xi_{\omega}\right)_{x}$ como o único vetor em $\left(L_{\omega}\right)_{x}$ satisfazendo $\omega_{x}\left(\xi_{\omega}\right)_{x}=1$.

Proposição 3.2 Se norma de $\omega$ é constante, então $\left(\xi_{\omega}\right)_{x}$ só depende de $\omega$ no ponto $x$.

\section{Prova:}

Suponhamos que $\tilde{\omega}$ é outra seçào de $\mathcal{D}^{0}$ passando pelo ponto $x, \operatorname{com} \omega_{x}=\tilde{\omega}_{x}$ e $\|\omega\|=\|\tilde{\omega}\|=c$ (onde $c$ denota uma constante). Afirmamos que, $\forall v \in \mathcal{D}_{x}$ tem-se $\left(i_{v} \text { od }(\omega-\widetilde{\omega})\right)_{x} \in \mathcal{D}_{x}^{0}$ e ortogonal a $\tilde{\omega}_{x}$. De fato, seja $\left\{\theta^{1}, \theta^{2}, \ldots, \theta^{d}\right\}$ referencial ortonormal local de $\mathcal{D}^{0}$ em torno de x. Então:

$$
\begin{array}{cc}
\omega=\sum_{i=1}^{d} f_{i} \theta^{i} & \tilde{\omega}=\sum_{i=1}^{d} g_{i} \theta^{i} \\
\sum_{i=1}^{d} f_{i}^{2}=\sum_{i=1}^{d} g_{i}^{2}=c & f_{i}(x)=g_{i}(x) \text { para } i=1,2, \ldots, d .
\end{array}
$$


Portanto:

$$
d \omega-d \tilde{\omega}=\sum_{i=1}^{d}\left(d f_{i}-d g_{i}\right) \wedge \theta^{i}+\left(f_{i}-g_{i}\right) d \theta^{i}
$$

Calculando em $x$ e aplicando $i_{v}$ temos:

$$
\left(i_{v} \circ d(\omega-\tilde{\omega})\right)=\sum_{i=1}^{d}\left[d f_{i}(v)-d g_{i}(v)\right] \theta^{i}
$$

Por outro lado, das equações (3.2) segue:

$$
\sum_{i=1}^{d} g_{i}(x)\left[d f_{i}(v)-d g_{i}(v)\right]=0
$$

que comparando com (3.1) e (3.3) é equivalente a:

$$
g_{x}^{0}\left(i_{v} \circ d(\omega-\tilde{\omega}), \tilde{\omega}\right)=0 .
$$

Assim, da proposição [3.1], usando a condiçào ii) temos que $d(\omega-\tilde{\omega})\left(\mathcal{D}_{x},\left(\xi_{\tilde{\omega}}\right)_{x}\right)=0$, e então da condição iii) $\left.\left(i_{\left(\xi_{\tilde{\omega}}\right)_{x}} \circ d \omega\right)\right|_{\mathcal{D}_{x}}=\left.\left(i_{\left(\xi_{\tilde{\omega}}\right)_{x}} \circ d \tilde{\omega}\right)\right|_{\mathcal{D}_{x}}=0$, e o resultado segue da unicidade de $\left(\xi_{\omega}\right)_{x}$.

Portanto, se $U \subset M$ é tal que $\omega_{x} \neq 0 \forall x \in U$, então fica definido um campo $\xi_{\omega}$ em $U$, dado por $\xi_{\omega}: x \in U \mapsto\left(\xi_{\omega}\right)_{x}$.

\section{Cálculo de $\xi_{\omega}$ em coordenadas}

Sejam $\left\{E_{i}, \tilde{E}_{\alpha}\right\}$ referencial local definido em $\mathcal{L}^{*} \subset M$ (restringindo $U$ se necessário) $\operatorname{com}\left\{E_{i}\right\}$ base ortonormal local de $\mathcal{D}$, e $\left\{\theta^{i}, \tilde{\theta}^{\alpha}\right\}$ o referencial dual associado. Então $\left\{\bar{\theta}^{i}=\left.\theta^{i}\right|_{\mathcal{D}}\right\}$ é base ortonormal de $\mathcal{D}^{*}$ e $\left\{\tilde{\theta}^{\alpha}\right\}$ é base de $\mathcal{D}^{0}$. Ortonormalizamos $\left\{\tilde{\theta}^{\alpha}\right\}$ e obtemos uma base ortonormal $\left\{\theta^{\alpha}\right\}$ de $\mathcal{D}^{0}$. Considere-se entào $\left\{E_{i}, E_{\alpha}=\xi_{\theta^{\alpha}}\right\}$ novo referencial definido em $U$.

$$
\begin{gathered}
\text { Temos: } \omega=\sum_{\alpha=n+1}^{n+d} a_{\alpha} \theta^{\alpha}\left(\text { pois } \omega \in \mathcal{D}^{0}\right) \quad . \quad \xi_{\omega}=\sum_{j=1}^{n} X^{j} E_{j}+\sum_{\beta=n+1}^{n+d} X^{\beta} E_{\beta} \\
\eta \in \mathcal{D}^{0} \quad \text { e } \eta \perp \omega \Longrightarrow \eta=\sum_{\alpha=n+1}^{n+d} b_{\alpha} \theta^{\alpha} \quad \text { e } \sum_{\alpha=n+1}^{n+d} a_{\alpha} b_{\alpha}=0 \\
\eta\left(\xi_{\omega}\right)=0 \quad \forall \quad \eta \perp \omega \Longrightarrow \sum_{\beta=n+1}^{n+d} b_{\beta} X^{3}=0 \quad \forall \quad b_{\beta} \quad \text { tal que } \sum_{\beta=n+1}^{n+d} a_{\beta} b_{\beta}=0 \\
\Longrightarrow X^{\gamma^{3}=\lambda} a_{3} \text { para } \beta=n+1, \ldots, n+d
\end{gathered}
$$




$$
\begin{aligned}
\omega\left(\xi_{\omega}\right)=1 & \Longrightarrow \sum_{\beta=n+1}^{n+d} a_{\beta} X^{\beta}=1 \Longrightarrow \sum_{\beta=n+1}^{n+d} a_{\beta}\left(\lambda a_{\beta}\right)=1 \Longrightarrow \lambda \sum_{\beta=n+1}^{n+d}\left(a_{\beta}\right)^{2}=1 \\
& \Longrightarrow \lambda\|\omega\|^{2}=1 \Longrightarrow \lambda=\frac{1}{\|\omega \cdot\|^{2}}
\end{aligned}
$$

Logo:

$$
X^{\beta}=\frac{a_{\beta}}{\|\omega\|^{2}} \quad \text { para } \quad \beta=n+1, \ldots, n+d
$$

Falta determinar $X^{j}$ para $j=1, \ldots, n$; para tal usaremos a condição $\left.\left(i_{\xi_{\omega}} \circ d_{\omega}\right)\right|_{\mathcal{D}}=0$. Temos:

$$
\begin{aligned}
d \omega & =\sum_{\alpha=n+1}^{n+d}\left(d a_{\alpha} \wedge \theta^{\alpha}+a_{\alpha} d \theta^{\alpha}\right) \\
i_{\xi_{\omega}} \circ d \omega & =\sum_{j=1}^{n} X^{j}\left(i_{E_{j}} \circ d \omega\right)+\sum_{\beta=n+1}^{n+d} X^{\beta}\left(i_{E_{\beta}} \circ d \omega\right) \\
& =\sum_{j=1}^{n} \sum_{\alpha=n+1}^{n+d}\left(X^{j} \frac{\partial a_{\alpha}}{\partial E_{j}} \theta^{\alpha}+X^{\gamma j} a_{\alpha}\left(i_{E_{j}} \circ d \theta^{\alpha}\right)\right)+ \\
& +\sum_{\alpha, \beta=n+1}^{n+d}\left(X^{\beta} \frac{\partial a_{\alpha}}{\partial E_{\beta}} \theta^{\alpha}-X^{\beta} \delta_{\alpha}^{\beta} d a_{\alpha}+X^{\beta} a_{\alpha}\left(i_{E_{\beta}} \circ d \theta^{\alpha}\right)\right)
\end{aligned}
$$

$$
\begin{aligned}
\left.\left(i_{\xi_{\omega}} \circ d \omega\right)\right|_{\mathcal{D}}=0 \Longrightarrow & \left.\sum_{j=1}^{n} \sum_{\alpha=n+1}^{n+d} X^{j} a_{\alpha}\left(i_{E_{j}} \circ d \theta^{\alpha}\right)\right|_{\mathcal{D}}-\left.\sum_{\alpha=n+1}^{n+d} X^{\alpha} d a_{\alpha}\right|_{\mathcal{D}} \\
& +\left.\sum_{\alpha, \beta=n+1}^{n+d} X^{\beta} a_{\alpha}\left(i_{E_{\beta}} \circ d \theta^{\alpha}\right)\right|_{\mathcal{D}}=0 \\
\Longrightarrow & \sum_{j=1}^{n}\left(\sum_{\alpha=n+1}^{n+d} a_{\alpha} d \theta^{\alpha}\left(E_{j}, E_{k}\right)\right) X^{j}=\sum_{\alpha=n+1}^{n+d} \frac{a_{\alpha}}{\|\omega\|^{2}} d a_{\alpha}\left(E_{k}\right) \\
& +\sum_{\alpha=n+1}^{n+d} \frac{a_{\alpha}}{\|\omega\|^{2}} d \theta^{\alpha}\left(E_{k}, \sum_{\beta=n+1}^{n+d} a_{\beta} E_{\beta}\right)
\end{aligned}
$$

$$
\forall \quad \mathrm{k}=1, \ldots \mathrm{n} \text {. }
$$

Supondo $\|\omega\|$ constante, temos $\sum_{\alpha=n+1}^{n+d} a_{\alpha}^{2}=c\left(\right.$ constante) e segue que $a_{\alpha} d a_{\alpha}\left(E_{k}\right)=0 \quad \forall k$. 
Assim, se ||$\omega||$ é constante a condição $\left.\left(i_{\xi_{\omega}} \circ d \omega\right)\right|_{\mathcal{D}}=0$ é equivalente à:

$$
\sum_{j=1}^{n} \omega\left(\left[E_{j}, E_{k}\right]\right) X^{j}=\sum_{\alpha=n+1}^{n+d} \frac{a_{\alpha}}{\|\omega\|^{2}} d \theta^{\alpha}\left(E_{k}, \sum_{\beta=n+1}^{n+d} a_{\beta} E_{\beta}\right) \quad \forall \quad k=1, \ldots, n .
$$

Da condição fortemente geradora por colchetes, temos que a matriz $\omega\left(\left[E_{j}, E_{k}\right]\right)$ é inversível, e portanto quando $\|\omega\|$ é constante os $X^{j}$ 's sào unicamente determinados como soluçào do sistema linear (3.5) dado acima.

Vamos agora apresentar a construção da métrica canônica associada a $M$, objetivo principal desta seção.

Consideremos a aplicação: $F: \mathcal{D}_{x}^{0}-\{0\} \rightarrow\left(T_{x} . M-\mathcal{D}_{x}\right)$

$$
\omega_{x} \longmapsto\left(\xi_{\omega}\right)_{x}
$$

Observamos que $F$ é nào linear e que, fixada uma base ortonormal $\left(\theta^{\alpha}\right)_{x}$ de $\mathcal{D}_{x}^{0}$, temos $d$ vetores $\left(\xi_{\theta^{\alpha}}\right)_{x}$ linearmente independentes em $\left(T_{x} M-\mathcal{D}_{x}\right)$. Podemos portanto definir um produto interno $g_{x}^{\theta^{\alpha}}$ em $T_{x} M$ : estabelecendo $\left(\xi_{\theta^{\alpha}}\right)_{x}$ ortonormais e perpendiculares à $\mathcal{D}_{x}$.

Agora, o espaço $S$ das bases ortonormais de $\mathcal{D}_{x}^{0}$ pode ser identificado com o grupo ortogonal compacto $O(d)$, através da aplicação: $A \in O(d) \longmapsto A .\left(\theta^{\star x}\right)_{x} \in S$.

Interessante seria se os $d$ vetores $\left(\xi_{\theta^{\alpha}}\right)_{x}$ e $\left(\xi_{A \cdot\left(\theta^{\alpha}\right)}\right)_{x}$ também mudassem por uma matriz ortogonal. Porém, em geral isso não é verdade. Para se certificar dessa afirmaçào basta observar que a restrição de $\left(\xi_{A \cdot\left(\theta^{\alpha}\right)}\right)_{x}$ à $\mathcal{D}_{x}$ está relacionada com a equação (3.5).

Assim, para se definir uma métrica intrínsica em $M$. consideremos a medida de Haar normalizada em $O(d)$ (a qual satisfaz $\mu(O(d))=1$ ) e definimos:

$$
<\cdot, \cdot>_{x}=\int_{O(d)} g_{x}^{A \cdot\left(\theta^{\alpha}\right)_{x}}(\cdot, \cdot) d \mu(A)
$$

A métrica Riemanniana $\langle\cdot, \cdot>$ definida como acima é tal que sua restrição à distribuição $\mathcal{D}$ coincide com a métrica $g$ e é denominada extensão canônica de $g$.

Sob a hipótese fortemente geradora por colchetes, temos que toda isometria sub-Riemanniana de $M$ é uma isometria Riemanniana na métrica Riemanniana canônica estendida sobre $M$. Assim, o grupo $G$ de todas as isometrias sub-Riemannianas de $M$ é um subgrupo (fechado) do grupo de todas as isometrias Riemannianas de $M$, portanto um grupo de Lie. Também o subgrupo de isotropia $K \subset G$ de um ponto $x_{0} \in M$ é um subgrupo de isotropia cujos elementos são isometrias Riemannianas, portanto compacto. 


\subsection{Espaços sub-simétricos e álgebras de Lie involuti- vas sub-ortogonais associadas}

Relembramos que um espaço sub-simétrico é uma variedade sub-Riemanniana $(M, \mathcal{D}, g)$ tal que para cada ponto $x_{0} \in M$ existe uma isometria sub-Riemanniana $\psi$, satisfazendo $\psi\left(x_{0}\right)=x_{0}$ e $\left.\psi_{\times}\right|_{\mathcal{D}_{x_{0}}}=-I d$.

Novamente, com argumento similar ao utilizado para as distribuições de Engel (Lema [2.2] e Corolário [2.1]) temos que a homogeneidade dos espaços sub-simétricos, no caso das distribuições fortemente geradora por colchetes, decorre da existência da sub-simetria em cada ponto. A compacidade do subgrupo de isotropia é, como observado anteriormente, consequência da extensào canônica da métrica sub-Riemanniana $g$. Finalmente, a condição $\psi^{2}=I d$ (onde $\psi$ é a sub-simetria) é demonstrada no lema abaixo.

Lema 3.1 Seja $(M, \mathcal{D}, g)$ variedade sub-Riemanniana, com $\mathcal{D}$ fortemente geradora por colchetes. Seja $x \in M$ e $\sigma: M \rightarrow M$ uma sub-isometria satisfazendo $\sigma(x)=x$ e $\left.\sigma_{*}\right|_{\mathcal{D}_{x}}=I d$, então $\sigma=I d_{M}$.

\section{Prova:}

Sejam $X, Y$ seções locais de $\mathcal{D}$ passando por $x$, então:

$$
\sigma_{*}([X, Y])=\left[\sigma_{*} X, \sigma_{*} Y\right] \Rightarrow \sigma_{*}\left([X, Y]_{x}\right)-[X, Y]_{x} \in \mathcal{D}_{x}
$$

Como, $T_{x} M=\mathcal{D}_{x}+\left\{\left[Z, X^{\prime}\right]_{x}: X\right.$ seção de $\left.\mathcal{D}\right\}$ para qualquer seção local $Z$ de $\mathcal{D}$ com $Z(x) \neq 0$, segue que $\sigma_{*}(\mathrm{v})-\mathrm{v} \in \mathcal{D}_{x}, \forall \mathrm{v} \in T_{x} M$. Por outro lado, usando a extensão canônica da métrica $g$, temos $T_{x} M=\mathcal{D}_{x} \Phi \mathcal{D}_{x}^{\perp}$ e $\sigma_{*}\left(\mathcal{D}_{x}^{\perp}\right)=\mathcal{D}_{x}^{\perp}$. Portanto, em particular, se $\mathrm{v} \in \mathcal{D}_{x}^{\perp}$ então $\sigma_{*}(\mathrm{v})-\mathrm{v} \in \mathcal{D}_{x}$ e $\sigma_{*}(\mathrm{v})-\mathrm{v} \in \mathcal{D}_{x}^{\perp}$, ou seja $\sigma_{*}(\mathrm{v})=\mathrm{v}$. Como $\sigma$ é uma isometria Riemanniana na métrica estendida. temos o resultado desejado.

Associaremos agora um objeto linear a um espaço sub-simétrico com distribuição fortemente geradora por colchetes. Para o caso particular de distribuições de contato os resultados desta seção encontram-se em [2].

No que segue $\mathcal{D}$ é uma distribuição de posto $n$ e codimensão $d$.

Teorema 3.1 Seja $(M, \mathcal{D}, g)$ um єspaço sub-simétrico com distribuição fortemente geradora por colchetes e $G$ o grupo de Lie de todas as isometrias sub-Riemannianas de M. Consideremos $x_{0} \in M$, seja $K$ o subgrupo de isotropia em $x_{0}$ e $\psi \in K$ a sub-simetria em $x_{0}$. Sejam 
$\mathfrak{g}$ e $\mathfrak{k}$ as respectivas álgebras de Lie de $G \in \mathbb{K}, \in$ finalmente seja $\mathfrak{g}=\mathfrak{h} \oplus \mathfrak{p}$ a decomposição de g nos \pm l-auto espaços da involução $s=A d_{\psi}$ de g. Então:

a. $M$ é representado como o espaço das classes $G / K$;

b. a projeção $\pi: G \rightarrow M$, dada por $\pi(g)=g\left(x_{0}\right)$, tem diferencial $\left.\left(\pi_{*}\right)\right|_{\mathfrak{p}}: \mathfrak{p} \cong \mathcal{D}_{x_{0}}$;

c. Ké uma subálgebra de $\mathfrak{h}$, compacta. de codimensão d que não contém ideais nào nulos de $\mathfrak{g}$ :

d. se $k \in K^{\prime}$ e $X \in \mathfrak{p}$, então $\pi_{*}\left(. A d_{k} X^{\prime}\right)=k_{*}\left(\pi_{*} X^{\prime}\right)$;

e. o produto interno $B$ induzido sobre $\mathfrak{p}$ pela identificação de $\mathfrak{p}$ com $\mathcal{D}_{x_{0}} \dot{e} \cdot \mathcal{A} d_{K^{-}}$-invariante,

f. $\forall X \in \mathfrak{p}$ o operador linear $\Theta_{X}: \mathfrak{p} \rightarrow \mathfrak{h} / \mathfrak{k}$ definido por $\Theta_{X}(Y)=[X, Y] \bmod \mathfrak{k} e ́$ sobrejetor.

\section{Prova:}

a. é imediata se observarmos que a ação de $G$ em $M$ é transitiva e preserva a estrutura sub-Riemanniana. Visto que a métrica sub-Riemanniana $g$ se estende canônicamente a uma métrica Riemanniana $\langle\cdot, \cdot\rangle$ sobre $M$ (vide seção [3.2]), $G$ é um grupo de isometrias Riemannianas relativas a métrica $\langle\cdot, \cdot\rangle$, e então $\mathrm{K}$ é compacto. Assim, existe uma decomposição $A d_{K}$-invariante $\mathfrak{g}=\mathfrak{k} \oplus \mathfrak{m}$. Consideremos a projeção $\pi: G \rightarrow M, \pi(g)=g\left(x_{0}\right)$. Temos que $\pi_{*}$

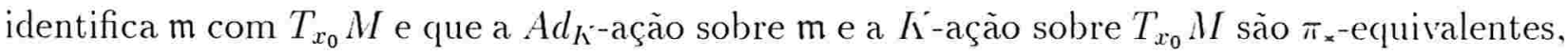
isto é, $\pi_{*}\left(A d_{k} X^{\prime}\right)=k_{*}\left(\pi_{*} X^{\prime}\right) \quad \forall k \in K, \forall X^{\prime} \in \mathfrak{m}$. Definimos $\mathfrak{p}_{0} \subset \mathfrak{m}$ como a imagem inversa de $\mathcal{D}_{x_{0}}$ através de $\pi_{*}$. Entào, $A d_{K}\left(\mathfrak{p}_{0}\right) \subset \mathfrak{p}_{0}$ e $\mathfrak{p}_{0} \subset \mathfrak{p}$, uma vez que $\left.\psi_{*}\right|_{\mathcal{D}_{x_{0}}}=-I d$. Segue-se que, o produto interno $g_{x_{0}}$ levantado para $\mathfrak{p}_{0}$ através de $\pi_{*}$, denotado por $B$, é $A d_{K^{-}}$-invariante. Também, segue-se que $\mathfrak{g}=\mathfrak{k}+\mathfrak{p}_{0}+\left[\mathfrak{p}_{0}, \mathfrak{p}_{0}\right]$ é uma decomposição $A d_{K}$-invariante, visto que $\mathcal{D}$ é uma distribuição fortemente geradora por colchetes. Agora, $A d_{K}$ é efetivo sobre m (porque $K$ é efetivo sobre $T_{x_{0}} M$ ), e isto implica que $A d_{K}$ é efetivo sobre $\mathfrak{p}_{0}$ (uma vez que $\mathfrak{g} / \mathfrak{k}$ é gerado por $\mathfrak{p}_{0}$ módulo $\mathfrak{k}$, devido à condição fortemente geradora por colchetes), ou equivalentemente que o centralizador de $\mathfrak{p}_{0}$ em $\mathfrak{k}$ é zero. Portanto, $\mathfrak{k}$ não contém ideais não nulos de $\mathfrak{g}$, pois se $\mathfrak{A} \subset \mathfrak{k}$ é um ideal de $\mathfrak{g}$ então $\mathfrak{A}$ está no centralizador de $\mathfrak{p}_{0}$ em $\mathfrak{k}$. Seja $X \in \mathfrak{k}$ and $Y \in \mathfrak{p}_{0}$, temos

$$
[X-s(X), Y]=[X, Y]-[s(X), Y]=[X, Y]+s([X, Y])=[X, Y]-[X, Y]=0
$$

e assim $a d_{X-s(X)}\left[\mathfrak{p}_{0}\right]=0, \operatorname{logo} \mathfrak{k} \subset \mathfrak{h}$. Portanto $\mathfrak{h}=\mathfrak{k}+\left[\mathfrak{p}_{\mathrm{o}}, \mathfrak{p}_{\mathrm{o}}\right]$ e $\mathfrak{p}_{0}=\mathfrak{p}$. Isso conclui a demonstração de b., c., d., e. Finalmente, $\Theta$ sobre $\mathfrak{p}$ é equivalente à $\mathcal{L}_{x_{0}}$ sobre $\mathcal{D}_{x_{0}} ;$ assim, f. segue da condição fortemente geradora por colchetes. 
Para um ponto $x_{0}$ de um espaço sub-simétrico com distribuiçào fortemente geradora por colchetes associamos uma quádrupla $(\mathfrak{g}, s, \mathfrak{k}, B)$. onde $\mathfrak{g}$ é uma álgebra de Lie real; $s$ é um automorfismo involutivo de $\mathfrak{g}$; $\mathfrak{k}$ é uma subálgebra de $\mathfrak{h}$ (auto espaço +1 de $s$ ), compacta. de codimensão $d$ e que não contém ideais não nulos de g e $B$ é um produto interno $a d_{\mathfrak{k}^{-}}$ invariante sobre $\mathfrak{p}$ (auto espaço -1 de $s$ ). Além disso. $\forall X \in \mathfrak{p}$ o operador $\Theta_{X}$ é sobrejetor. A quádrupla $(\mathfrak{g}, s, \mathfrak{k}, B)$ é denominada álgebra de Lie incolutiva sub-ortogonal (LISO-álgebra) de $M$ em $x_{0}$. Como $M$ é homogêneo, suas LISO-álgebras são as mesmas em todos os pontos

Uma LISO-álgebra abstrata é uma quádrupla ( g, s, k $B$ ) com as propriedades do parágrafo anterior. Uma subálgebra de Lie involutiva sub-ortogonal (LISO-subálgebra) de (g, s, k. B) é uma LISO-álgebra $\left(\mathfrak{g}^{\prime}, s^{\prime}, \mathfrak{k}^{\prime}, B^{\prime}\right)$ tal que $\mathfrak{g}^{\prime}$ é uma subálgebra de $\mathfrak{g}, s^{\prime}$ é a restrição de $s$ à $\mathfrak{g}^{\prime}$, $\mathfrak{k}^{\prime}$ é uma subálgebra de $\mathfrak{k}, \mathfrak{p}^{\prime}=\mathfrak{p}$ e $B^{\prime}=B$.

Dado uma LISO-álgebra abstrata $(\mathfrak{g}, s, \mathfrak{k}, B)$ podemos construir um espaço sub-simétrico. simplesmente conexo, com distribuiçào fortemente geradora por colchetes, como segue. Sejam $\widetilde{G}$ o grupo de Lie simplesmente conexo com álgebra de Lie $\mathfrak{g}, \mathfrak{g}=\mathfrak{h} \oplus \mathfrak{p}$ a decomposiçào de $\mathfrak{g}$ nos \pm 1 -auto-espaços de $s$ e $\widetilde{K} \subset \widetilde{G}$ o subgrupo conexo associado à subálgebra $\mathfrak{k} \subset \mathfrak{g}$. Consideremos a variedade simplesmente conexa $. M=\widetilde{G} / \widetilde{K}$ e a projeção $\pi: \widetilde{G}_{t} \rightarrow M$ dada por $\pi(g)=g \widetilde{K}=g\left(x_{0}\right)$, onde $x_{0}$ é a classe $\widetilde{K}$. Então o subespaço $\mathcal{D}_{x_{0}}=\pi_{*}(\mathfrak{p}) \subset T_{x_{0}} M$ e o produto interno $B$-projetado para $\mathcal{D}_{x_{0}}$ através de $\pi_{*}$, denotado por $g_{x_{0}}$, são $\widetilde{K^{-}}$-invariantes, visto que $\pi_{\times}\left(A d_{k} X\right)=\left(d L_{k}\right)_{x_{0}}\left(\pi_{*} X\right) \quad \forall k \in \widetilde{\Lambda^{\prime}}, \forall X \in \mathfrak{p}$, onde $L_{k}: M \rightarrow M, L_{k}\left(g \widetilde{K^{\prime}}\right)=k g \widetilde{\Lambda^{\prime}}$. Seja $\mathcal{D}$ a distribuição $\tilde{C}_{\boldsymbol{i}}$-invariante sobre $M$ determinada por $\mathcal{D}_{x_{0}}$. A distribuição $\mathcal{D}$ assim definida é fortemente geradora por colchetes, pois o operador linear $\Theta_{X}$ é sobrejetor $\forall X \in \mathfrak{p}$. Seja $g$ a métrica $\widetilde{G}_{t}$-invariante sobre $\mathcal{D}$ determinada por $g_{x_{0}}$. O automorfismo $s$ de $\mathfrak{g}$ induz um automorfismo $\varphi$ de $\tilde{G}$ tal que $\varphi_{*}=s$, o qual se estende a uma transformação $\psi: M \rightarrow M$ definida por $\psi\left(g \widetilde{K^{\prime}}\right)=\varphi(g) \widetilde{K^{\prime}}$. Então $\psi$ é uma isometria sub-Riemanniana de $M$ relativa à métrica sub-Riemanniana $g$ e também $\psi_{*}\left(\pi_{*} X\right)=\pi_{*}\left(s X^{\prime}\right)=-\pi_{*} X \quad \forall X \in \mathfrak{p}$, assim $\psi$ fixa $x_{0}$ e $\psi_{*}$ é -1 sobre $\mathcal{D}_{x_{0}}$. Portanto $\psi$ é a sub-simetria em $x_{0}$ e $\phi_{g\left(x_{0}\right)}: M \rightarrow M$ definida por $\phi_{g\left(x_{0}\right)}(x)=g \psi g^{-1}(x)$ é a sub-simetria em $g\left(x_{0}\right)$. Provamos então que $M$ é sub-simétrico com distribuição fortemente geradora por colchetes.

Proposição 3.3 Seja $(\mathfrak{g}, s, \mathfrak{k}, B)$ uma LISO-álgebra abstrata. Seja M o espaço sub-simétrico com distribuição fortemente geradora por colchetes, simplesmente conexo, construido à partir de $(\mathfrak{g}, s, \mathfrak{k}, B)$. Então $(\mathfrak{g}, s, \mathfrak{k}, B)$ é uma LISO-subálgebra da LISO-álgebra associada à $M$.

\section{Prova:}

De fato, sejam $\widetilde{G}$ e $\widetilde{K}$ os grupos de Lie construidos a partir da LISO-álgebra abstrata $(\mathfrak{g}, s, \mathfrak{k}, B)$, como no parágrafo anterior. Seja $G^{\prime}$ o grupo de Lie de todas as isometrias 
sub-Riemannianas de $M=\widetilde{G} / \widetilde{K^{\prime}}, K^{\prime \prime} \subset G^{\prime}$ o subgrupo de isotropia em $x_{0} \in M$ (onde $x_{0}$ é a classe $\left.\widetilde{K^{\prime}}\right)$ e construimos a LISO-álgebra associada $\left(\mathfrak{g}^{\prime}, s^{\prime}, \mathfrak{k}^{\prime}, B^{\prime}\right)$. Consideremos o homomorfismo de grupos $f: \tilde{G}_{i} \rightarrow C_{r}^{\prime \prime}$ dado por $f(g)=\bar{L}_{g}: M \rightarrow M, \bar{L}_{g}(x)=g . x \quad \forall x \in M$. Como $G^{\prime}$ possui a topologia compacto-aberta, temos que $f$ é contínuo $\left(\mathcal{C}^{\infty}\right)$, pois um aberto em $G^{\prime \prime}$ é da forma $\mathcal{A}(\mathrm{C}, \mathrm{U})=\left\{g^{\prime} \in G^{\prime} / g^{\prime}(\mathrm{C}) \subset \mathrm{U}\right\}$ onde $\mathrm{C} \subset M=\widetilde{G} / \widetilde{K^{\prime}}$ é compacto e $\mathrm{U} \subset . M$ é aberto e assim $f^{-1}\left(\mathcal{A}\left(C . C^{-}\right)\right)=\left\{\tilde{g} \in \tilde{G} / \bar{L}_{\tilde{g}}(C) \subset \mathrm{U}\right\}$ é aberto, uma vez que a açào de $\tilde{G}$ em $M$ é contínua. Denotamos por $\widetilde{Z}$ o núcleo da aplicaçào $f$ e observamos que $\tilde{Z}$ está contido em $\widetilde{K}$. Temos que $G_{0}^{\prime}=\widetilde{G} / \tilde{Z}$ é isomorfo ao subgrupo $f(\tilde{G}) \subset G^{\prime}$ e $\kappa_{0}=\widetilde{K^{\prime}} / \tilde{Z}$ é isomorfo ao subgrupo $f\left(\widetilde{K^{-}}\right) \subset \Lambda^{-1}$. Visto que $\mathfrak{k}$ nào contém ideais não nulos de $\mathfrak{g}, \widetilde{Z}$ é discreto, assim $\mathfrak{g}$ e $\mathfrak{k}$ são as álgebras de Lie de $G_{0}$ e $K_{0}$, e temos as inclusões de subálgebras de Lie $\mathfrak{g} \subset \mathfrak{g}^{\prime}$ e $\mathfrak{k} \subset \mathfrak{k}^{\prime}$. Também o diagrama comutativo:

\begin{tabular}{|c|c|c|c|c|c|c|}
\hline$G_{0}$ & $c$ & $G^{\prime}$ & & & & \\
\hline$\pi \downarrow$ & & $\downarrow \pi^{\prime}$ & & $p$ & $\longrightarrow$ & $\mathfrak{p}^{\prime}$ \\
\hline$\tilde{G} / \widetilde{K^{\prime}} \cong G_{0} / K_{0}$ & & $G^{\prime} / K^{\prime}$ & induz & $\pi_{*} \downarrow \cong$ & & $\cong \downarrow \pi_{*}^{\prime}$ \\
\hline$\|$ & & $\|$ & & $\mathcal{D}_{x_{0}}$ & $\overrightarrow{I d}$ & $\mathcal{D}_{x_{0}}$ \\
\hline .11 & $\overrightarrow{I d}$ & $M$ & & & & \\
\hline
\end{tabular}

Portanto $\mathfrak{p}=\mathfrak{p}^{\prime}, s^{\prime}=\left.s\right|_{\mathfrak{g}^{\prime}}$ e $B=B^{\prime}$.

A importância deste resultado decorre da

Proposição 3.4 Sejam $(\mathfrak{g}, s, \mathfrak{k}, B) \subset\left(\mathfrak{g}^{\prime}, s^{\prime}, \mathfrak{k}^{\prime}, B^{\prime}\right)$ LISO-álgebras, sejam $M$ e $M^{\prime}$ os espaços sub-simétricos, simplesmente conexos, com distribuições fortemente geradoras por colchetes correspondentes. Então . I e $M^{\prime}$ são isométricos.

\section{Prova:}

Sejam $\mathfrak{g}=\mathfrak{h}+\mathfrak{p}$ e $\mathfrak{g}^{\prime}=\mathfrak{h}^{\prime}+\mathfrak{p}^{\prime}$ sob $s$ e $s^{\prime}$, respectivamente. Denotemos $M=\widetilde{G} / \widetilde{K^{\prime}}$ e $M^{\prime}=\widetilde{G}^{\prime} / \widetilde{L^{\prime}}$, onde $\tilde{G}$ e $\widetilde{G}_{r}^{\prime}$ são os grupos simplesmente conexos determinados por $\mathfrak{g}$ e $\mathfrak{g}^{\prime}$. Integrando a inclusào $\mathfrak{g} \subset \mathfrak{g}^{\prime}$ obtemos $f: \tilde{G} \rightarrow \tilde{G}^{\prime}$ homomorfismo com núcleo discreto. Como $\operatorname{Lie}\left(\widetilde{K^{\prime}}\right)=\mathfrak{k} \subset \mathfrak{k}^{\prime}=\operatorname{Lie}\left(\widetilde{K^{\prime \prime}}\right)$ temos $f\left(\widetilde{K^{\prime}}\right) \subset \widetilde{\Lambda^{\prime \prime}}$. Assim, fica induzida uma aplicaçào $\bar{f}: M=\widetilde{G^{\prime}} / \widetilde{K^{\prime}} \rightarrow M^{\prime}=\widetilde{G^{\prime}} / \widetilde{K^{\prime \prime}}$ dada por $\bar{f}\left(\tilde{g} \widetilde{K^{\prime}}\right)=f(\tilde{g}) \widetilde{K^{\prime \prime}}$. A aplicação $\bar{f}$ é sobrejetora, pois $M=(\exp \mathfrak{m})\left(x_{0}\right), M^{\prime}=(\exp \mathrm{m})\left(x_{0}^{\prime}\right)$ e $f(\exp \mathrm{m})=\exp \mathbf{m}$, onde $\mathbf{m}=\mathfrak{p}+[\mathfrak{p}, \mathfrak{p}] \subset \mathfrak{g} \subset \mathfrak{g}^{\prime}$, 
$x_{0}$ e $x_{0}^{\prime}$ sào as classes $\widetilde{K^{\prime}}$ e $\widetilde{\Lambda^{\prime \prime}}$. Agora $M^{\prime}=\widetilde{G}^{\prime \prime} / \widetilde{K^{\prime \prime}}$ onde $\widetilde{G}^{\prime \prime \prime}=f(\widetilde{G})$. $\widetilde{K^{\prime \prime}}=f(\widetilde{G}) \cap \widetilde{K^{\prime \prime}}$ e entào $f\left(\widetilde{K^{\prime}}\right) \subset \widetilde{K^{\prime \prime}}$ e $\bar{f}: \widetilde{G} / \widetilde{K^{\prime}} \rightarrow \widetilde{G}^{\prime \prime \prime} / \widetilde{\Lambda^{\prime \prime}}$. Visto que o núcleo do homomorfismo $f$ é discreto, $\operatorname{Lie}\left(\tilde{G}^{\prime \prime}\right)=\operatorname{Lie}(\widetilde{G})=\mathfrak{g e} \operatorname{Lie}\left(f\left(\widetilde{\Pi^{\prime}}\right)\right)=\operatorname{Lie}\left(\widetilde{K^{\prime}}\right)=\mathfrak{k} \subset \operatorname{Lie}\left(\widetilde{K^{\prime \prime}}\right)$. Mas.

$$
\operatorname{dim} \widetilde{K^{\prime \prime}}=\operatorname{dim} \widetilde{G^{\prime \prime \prime}}-\operatorname{dim} M^{\prime}=\operatorname{dim} f(\widetilde{G})-\left[\operatorname{dim}\left(\mathfrak{p}^{\prime}\right)+d\right]=\operatorname{dim} \widetilde{G}-\operatorname{dim} M=\operatorname{dim} \widetilde{K^{\prime}}
$$

portanto Lie $\left(\widetilde{K^{\prime \prime}}\right)=\mathfrak{k}$. Logo, o diagrama comutativo

$$
\begin{aligned}
& \tilde{G}^{\prime} \stackrel{f}{\longrightarrow} \tilde{G}^{\prime \prime \prime} \\
& \mathfrak{g} \stackrel{I d}{\longrightarrow} \quad \mathfrak{g} \\
& \pi \downarrow \quad \downarrow \pi^{\prime} \quad \text { induz } \quad \pi_{*} \downarrow \quad \downarrow \pi_{*}^{\prime} \\
& M=\tilde{G} / \widetilde{K^{\prime}} \underset{\bar{f}}{\longrightarrow} \tilde{G^{\prime \prime}} / \widetilde{K^{\prime \prime \prime}}=M^{\prime} \quad \mathfrak{g} / \mathfrak{k} \underset{(d \bar{f})_{x_{0}}}{\longrightarrow} \mathfrak{g} / \mathfrak{k}
\end{aligned}
$$

e então $(d \bar{f})_{x_{0}}=I d$. Portanto $\bar{f}$ é difeomorfismo local em $x_{0}$, como $\mathfrak{p}=\mathfrak{p}^{\prime}$ e $B=B^{\prime}$ segue que $\bar{f}$ é isometria sub-Riemanniana local em $x_{0}$. Agora $\bar{f}$ é equivariante (isto é, $\bar{f} \circ L_{\tilde{g}}=L_{f(\bar{g})}^{\prime} \circ \bar{f}$, $\forall \check{g} \in \widetilde{G}^{\prime}$, onde $L_{\tilde{g}}: M \rightarrow M$ e $L_{f(\tilde{g})}^{\prime}: M^{\prime} \rightarrow M^{\prime}$ são as translaçòes à esquerda), $B$ e p são $a d_{\mathfrak{k}}$-invariantes e as estruturas sub-Riemannianas de $M$ e $M^{\prime}$ são as transladadas de $B$ e $\mathfrak{p}$, $\operatorname{assim} \bar{f}$ é isometria sub-Riemanniana local em todos os pontos. Sendo $M$ e $M^{\prime}$ completas temos que $\bar{f}$ é um recobrimento. Como $M^{\prime}$ é simplesmente conexa, $\bar{f}$ é difeomorfismo global, portanto isometria global.

No sentido oposto, podemos enunciar a

Proposição 3.5 Sejam M um espaço sub-simétrico, simplesmente conexo, com distribuição fortemente geradora por colchetes, $(\mathfrak{g}, s, \mathfrak{k}, B)$ a LISO-álgebra associada e $\widetilde{M}$ o espaço sub-

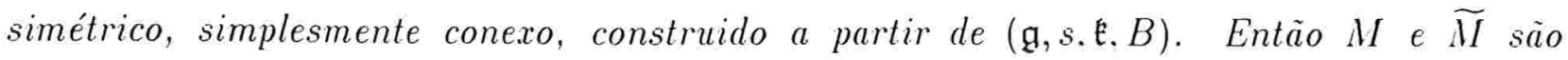
isométricos.

\section{Prova:}

De fato, denotemos $M=G / K$ e $\widetilde{M}=\widetilde{G} / \widetilde{K^{T}}$ onde $G$ é o grupo de Lie de todas as isometrias sub-Riemannianas de $M, \Lambda$ é o subgrupo de isotropia em $x_{0} \in M$, $\tilde{G}_{r}$ é o grupo de Lie simplesmente conexo com álgebra de Lie g e $\widetilde{K} \subset \widetilde{G}$ é o grupo conexo definido por $\mathfrak{k} \subset \mathfrak{g}$. Como $\widetilde{G}_{i}$ é simplesmente conexo e Lie $(\widetilde{G})=\mathfrak{g}=\operatorname{Lie}\left(G_{r}\right)$, existe um recobrimento $r: \widetilde{G}_{i} \rightarrow G_{r}$ tal que $r_{\star}=I d$. Também Lie $\left(\widetilde{K^{*}}\right)=\mathfrak{k}=\operatorname{Lie}\left(\kappa^{\prime}\right)$ implica que $r\left(\widetilde{K^{\prime}}\right) \subset K$, assim $r$ induz uma 
aplicação sobrejetora $\bar{r}: \widetilde{M} \rightarrow M$. dada por $\bar{r}\left(\tilde{g} \widetilde{K^{r}}\right)=r(\tilde{g}) K$. Temos $\mathfrak{g}=\mathfrak{h} \oplus \mathfrak{p}$ sob s e o diagrama comutativo

$$
\begin{aligned}
& \widetilde{G} \stackrel{r}{\longrightarrow} G \\
& \mathfrak{g} \stackrel{I d}{\longrightarrow} \mathfrak{g} \\
& \tilde{\pi} \downarrow \quad \downarrow \pi \\
& \text { induz } \\
& \tilde{\pi}_{x} \downarrow \\
& \downarrow \pi_{x} \\
& \widetilde{M}=\tilde{G} / \widetilde{K} \longrightarrow G / K=M \\
& \mathfrak{g} / \mathfrak{k} \quad \longrightarrow \quad \mathfrak{g} / \mathfrak{k} \\
& \bar{r} \\
& (d \bar{r})_{\tilde{x}_{0}}
\end{aligned}
$$

portanto $(d \bar{r})_{\tilde{x}_{0}}=I d$, onde $\tilde{x}_{0}=1 \widetilde{K}$. Como o produto interno em $\tilde{\pi}_{*}(\mathfrak{p})$ e em $\pi_{\times}(\mathfrak{p})$ são os mesmos temos que $\bar{r}$ é isometria sub-Riemanniana local em $\tilde{x}_{0}$. Agora $\bar{r}$ é equivariante, e as estruturas sub-Riemannianas de $M$ e $\widetilde{M}$ sào preservadas pelas respectivas açòes de $G$ e $\tilde{G}$, então segue que $\bar{r}$ é isometria sub-Riemanniana local em todos os pontos. Mas $M$ e $\widetilde{M}$ são homogêneas. portanto completas, assim $\bar{r}$ é um recobrimento sub-Riemanniano. Finalmente, $M$ é simplesmente conexo, $\bar{r}$ é isometria global.

Para facilitar os cálculos temos a

Proposição 3.6 Se $(\mathfrak{g}, s, \mathfrak{k}, B)$ é uma LISO-álgebra então ela admite uma LISO-subálgebra $\left(\mathfrak{g}^{\prime}, s^{\prime}, \mathfrak{k}^{\prime}, B^{\prime}\right)$ tal que $\left[\mathfrak{p}^{\prime}, \mathfrak{p}^{\prime}\right]=\mathfrak{h}^{\prime}$.

\section{Prova:}

Seja $\mathfrak{g}=\mathfrak{h}+\mathfrak{p}$ sob $s$. Consideremos $\mathfrak{g}^{\prime}=\mathfrak{p}+[\mathfrak{p}, \mathfrak{p}]$ (é claro que $\mathfrak{g}^{\prime}$ assim definida é uma subálgebra de $\mathfrak{g}$, na verdade, um ideal) e definimos $\mathfrak{k}^{\prime}=\mathfrak{k} \cap(\mathfrak{p}+[\mathfrak{p}, \mathfrak{p}])=\mathfrak{k} \cap[\mathfrak{p}, \mathfrak{p}], \mathfrak{p}^{\prime}=\mathfrak{p}$, $s^{\prime}=\left.s\right|_{\mathfrak{g}^{\prime}}$ e $B^{\prime}=B$.

\subsection{Métrica canônica no caso sub-simétrico}

Para os espaços sub-simétricos podemos caracterizar a métrica canônica, definida pela relação (3.6), em termos da sua estrutura de LISO-álgebra. Com esta finalidade apresentamos na sequência duas proposições e um lema preliminares.

Proposição 3.7 Seja $(\mathfrak{g}, s, \mathfrak{k}, B)$ LISO-álgebra abstrata com $\mathfrak{g}=[\mathfrak{p}, \mathfrak{p}] \oplus \mathfrak{p}$, então o produto interno ad $d_{\mathfrak{k}}$-invariante $B$, definido sobre $\mathfrak{p}$, se estende intrinsicamente a um produto interno ad $d_{\mathfrak{k}}$-invariante $\bar{B}$ sobre g. 


\section{Prova:}

Sejam $\mathfrak{p}^{*}$ e $[\mathfrak{p}, \mathfrak{p}]^{*}$ os espaços vetoriais duais de $\mathfrak{p}$ e $[\mathfrak{p}, \mathfrak{p}]$, respectivamente.

Consideremos a aplicação $j:[\mathfrak{p}, \mathfrak{p}]^{\times} \longrightarrow \mathfrak{p}^{*} \wedge \mathfrak{p}^{*}$

$$
\rho \quad \longmapsto \rho([\cdot, \cdot])
$$

Temos que $j$ é injetora e $a d_{\mathfrak{k}}$-invariante (ie, $j \circ a d_{\mathfrak{k}}=a d_{\mathfrak{k}} \circ j$ ). Como um produto interno $\operatorname{ad}_{\mathfrak{k}}$ invariante sobre $\mathfrak{p}$ induz naturalmente $u m$ produto interno ad $d_{\mathfrak{k}}$-invariante sobre $\mathfrak{p}^{\times} \wedge \mathfrak{p}^{*}$, este produto interno pode ser $j$-levantado para $[\mathfrak{p}, \mathfrak{p}]^{*}$. Assim, temos um produto interno $\operatorname{ad}_{\mathfrak{k}}$-invariante definido em $[\mathfrak{p}, \mathfrak{p}]^{*}$. Como $\mathfrak{g}^{\times}=[\mathfrak{p}, \mathfrak{p}]^{\times} \oplus \mathfrak{p}^{*}$ o resultado segue.

Proposição 3.8 Sejam $(\mathfrak{g}, s, \mathfrak{k}, B)$ LISO-álgebra abstrata com $\mathfrak{g}=[\mathfrak{p}, \mathfrak{p}] \oplus \mathfrak{p}, \mathfrak{k}^{\perp}$ o complemento $\bar{B}$-ortogonal de $(\mathfrak{k} \oplus \mathfrak{p})$ em $\mathfrak{g} . \mathfrak{m}=\mathfrak{k}^{\perp} \pm \mathfrak{p} \in \pi \mathfrak{m}: \mathfrak{g}=\mathfrak{k} \oplus \mathfrak{m} \rightarrow \mathfrak{m}$ a projeçào. Então. dado $\omega \neq 0 \in \mathfrak{p}^{0} \subset \mathfrak{m}^{*}$. existe um único l'etor $\left(\xi^{\omega}\right) \in \mathfrak{m}=\mathfrak{k}^{\perp} \oplus \mathfrak{p}$ tal que :

i) $\omega\left(\xi^{\omega}\right)=1$;

ii) $\eta\left(\xi^{\omega}\right)=0 \quad \forall \eta \perp \omega \quad\left(\eta \in \mathfrak{p}^{0}\right)$;

iii) $\omega\left(\pi_{\mathfrak{m}}\left[\xi^{\omega}, \mathrm{v}\right]\right)=0, \quad \forall \mathrm{v} \in \mathfrak{p}$.

Além disso, a condição iii) $\omega\left(\pi_{\mathfrak{m}}\left[\xi^{\omega}, \mathrm{v}\right]\right)=0, \quad \forall \mathrm{v} \in \mathfrak{p} \quad$ é equivalente a $\xi^{\omega} \in \mathfrak{k}^{\perp}$.

\section{Prova:}

A existência e unicidade de $\left(\xi^{\omega}\right)$ pode ser demonstrada da mesma maneira que na prova da proposição 3.1 , substituindo naquela demonstração $T_{x} M$ por $\mathfrak{m}, \mathcal{D}_{x}^{0}$ por $\mathfrak{p}^{0},(J \omega)_{x}\left(\mathcal{D}_{x}\right)$ por $\left\{\omega\left(\pi_{\mathfrak{m}}[\mathrm{v}, \cdot]\right): \mathfrak{m} \rightarrow \mathbb{R} / \mathrm{v} \in \mathfrak{p}\right\}$. A equivalência entre iii) e a condição $\xi^{\omega} \in \mathfrak{k}^{\perp}$. prova-se como segue: seja $\xi^{\omega} \in \mathfrak{m}, \xi^{\omega}=\xi_{1}+\xi_{2} \operatorname{com} \xi_{1} \in \mathfrak{k}^{\perp}$ e $\xi_{2} \in \mathfrak{p}$. Temos, $\omega\left(\pi_{\mathfrak{m}}\left[\xi^{\omega}, \mathrm{v}\right]\right)=0, \forall \mathrm{v} \in \mathfrak{p}$ se, e somente se $\omega\left(\pi_{\mathfrak{m}}\left[\xi_{2}, v\right]\right)=0, \forall \mathrm{v} \in \mathfrak{p}$, uma vez que $\omega \in \mathfrak{p}^{0}$ e $\left[\xi_{1}, v\right] \in \mathfrak{p} \forall v \in \mathfrak{p}$. Agora, da hipótese fortemente geradora por colchetes temos: se $\xi_{2} \neq 0$ então $\left\{\pi \mathfrak{m}\left[\xi_{2}, v\right]: v \in \mathfrak{p}\right\} \oplus \mathfrak{p}=\mathfrak{m}$. Portanto, $\omega \neq 0 \in \mathfrak{p}^{0} \subset \mathrm{m}^{*}$ e $\omega\left(\pi_{\mathfrak{m}}\left[\xi_{2}, v\right]\right)=0 \forall \mathrm{v} \in \mathfrak{p}$ implica que $\xi_{2}=0$.

O próximo lema juntamente com sua demonstração encontra-se em [12].

Lema 3.2 Sejam $G$ um grupo de Lie e $K \subset G$ um subgrupo fechado, com álgebras de Lie $\mathfrak{g}$ e k respectivamente. Seja $\mathrm{m} \subset \mathfrak{g}$ um subespaço complementar de $\mathfrak{k}$ em $\mathfrak{g}$ e seja $\mathrm{s}: U \subset$ $G / K \rightarrow G$ uma seção local tal que $1 K \in U . \mathrm{s}(1 \mathrm{~K})=1$ e tal que a imagem de $(d \mathrm{~s})_{1 K}$ seja m. Para $\mathrm{v} \in \mathfrak{g}$ definimos um campo $\vec{V}$ em $U$ fazendo $\vec{V}_{g K}=\left(d \bar{L}_{\mathrm{s}(\mathrm{gK})}\right)_{1 K}\left(\pi_{\times}(\mathrm{v})\right)$, onde $\pi: G \rightarrow G / K$ é a projeção e $\bar{L}_{\mathrm{s}(\mathrm{gK})}: G / K \rightarrow G / K$ é a translação à esquerda por $\mathrm{s}(\mathrm{g} K)$. Então para $\mathrm{v}, \mathrm{w} \in \mathrm{m}$ temos $\left[\vec{V}, \overrightarrow{W^{*}}\right]_{1 K^{\circ}}=\overrightarrow{[V, W]_{1 K}}=\pi_{*}([\mathrm{v}, \mathrm{w}])$. 


\section{Prova:}

Seja $g H^{*} \in L^{*}$ e suponha $g=s\left(g K^{*}\right)$. Diferenciando a identidade $\pi \circ L_{g}=\bar{L}_{g} \circ \pi$ no ponto 1 e aplicando sobre $u m$ vetor $\mathrm{v} \in \mathfrak{g}$ concluímos que:

$$
\vec{V}_{g K}=d \pi_{g}\left(V_{g}^{L}\right)
$$

onde $V^{L}$ é o campo invariante à esquerda tal que $V_{1}^{L}=v$.

Seja $f: U \rightarrow \mathbb{R}$ uma funçào. Basta mostrar que $[\vec{V}, \vec{W}]_{1 K}$ e $\left.\overrightarrow{[V, W}\right]_{1 K}=\pi_{x}([v, w])$ coincidem quando aplicados sobre $f$ (como derivadas direcionais). Segue de (3.7) que:

$$
\vec{V}(f)=V^{L}(f \circ \pi) \circ s .
$$

Como $\pi_{*} \circ d s_{1 K}=I d$ e $\mathrm{w} \in \mathfrak{m}$ temos $d s_{1 \kappa} \circ \pi_{*}(\mathrm{w})=\mathrm{w}$. Aplicando o vetor $\vec{W}_{1 K}=\pi_{*}(\mathrm{w})$ nos dois lados de (3.8) vem:

$$
\vec{W} \vec{V}(f)_{1 \kappa}=W^{-L} V^{L}(f \circ \pi)_{1} .
$$

Trocando os papéis de v e w em (3.9) e subtraindo as duas equações obtidas segue o resultado desejado.

Observação 3.1 Sempre existem seções locais como as enunciadas no lema (3.2). Podemos. por exemplo. considerar a inversa da restrição de $\pi$ à imagem pela exponencial de um aberto suficientemente pequeno contendo a origem $\mathrm{em} \mathrm{m}$.

Enunciamos agora o resultado principal desta seção

Teorema 3.2 Seja $(\mathfrak{g}, s, \mathfrak{k}, B)$ uma LISO-álgebra abstrata com $\mathfrak{g}=[\mathfrak{p}, \mathfrak{p}] \oplus \mathfrak{p}$ e $M=G / K$ o espaço sub-simétrico, simplesmente conexo, associado. Então, a métrica canônica definida em $M=G / K$ é a métrica $G$-invariante determinada no ponto base pelo produto interno $\bar{B}$ projetado através de $\pi_{*}$.

\section{Prova:}

Como $G / K$ é homogêneo basta verificar que estas métricas coincidem no ponto base. Consideramos a projeção $\pi: G \rightarrow G / K$ e temos que $\pi_{x}$ identifica $\mathfrak{m}=\mathfrak{k}^{\perp} \oplus \mathfrak{p} \operatorname{com}(\mathfrak{g} / \mathfrak{k})=T_{x_{0}} . M$ (onde $x_{0}$ é classe $1 K$ ) e $\mathfrak{p}$ com $D_{x_{o}}$. Com esta identificaçào $\pi_{*}$ corresponde à $\pi_{\mathrm{m}}$ e o produto interno canônico definido em $\mathcal{D}_{x_{0}}^{0} \equiv \mathfrak{p}^{0} \subset \mathfrak{m}^{*}$. denotado por $g_{x_{0}}^{0}$, corresponde ao produto interno induzido de $\mathfrak{p}^{*} \wedge \mathfrak{p}^{*}$ através da aplicação injetora $J: \mathfrak{p}^{0} \rightarrow \mathfrak{p}^{*} \wedge \mathfrak{p}^{*}, J(\rho)=\rho(\pi \mathfrak{m}[\cdot, \cdot])$. 
Agora, dado $\omega_{x_{0}} \in\left(T_{x_{0}}, \mathcal{H}\right)^{\times} \equiv(\mathfrak{g} / \mathfrak{k})^{\times} \operatorname{com} \omega_{x_{0}}\left(\mathcal{D}_{x_{0}}\right)=0$. podemos estendê-lo localmente em $\mathcal{D}^{0}$ com norma constante de uma maneira conveniente: Escolhemos $s: U \subset G / K \rightarrow G$ seção local com $1 K \in U, s\left(1 K^{\circ}\right)=1$ e tal que a imagem de $(d s)_{1 K^{\circ}}$ seja $\mathrm{m}$. Definimos $\vec{\omega}$ em $U$ por $\vec{\omega}\left(g K^{\prime}\right)=\left(\bar{L}_{s\left(g K^{\circ}\right)}{ }^{-1}\right)^{*} \omega$. onde $\bar{L}_{s\left(g K^{\circ}\right)}: M \rightarrow M$ é a translaçào à esquerda, temos que $\vec{\omega}$ está em $\mathcal{D}^{0}$ e $\|\vec{\omega}\|$ é constante em $U$, pois a ação de $G$ é por isometrias sub-Riemannianas. Dados, $\mathrm{v}, \mathrm{w} \in \mathrm{m}$ temos: $d \overrightarrow{\mathrm{u}}(\vec{V}, \vec{W})=-\vec{w}([\vec{V}, \vec{W}])$, onde $\vec{V} \cdot \vec{W}$ são construidos como no lema (3.2). Logo, no ponto base, tem-se :

$$
d \vec{\omega}_{x_{0}}(\mathrm{v}, \mathrm{w})=-\omega_{x_{0}}\left([\vec{V}, \vec{W}]_{x_{0}}\right)=-\omega_{x_{0}}\left(\pi_{\times}[\mathrm{v}, \mathrm{w}]\right) \equiv-\omega_{x_{0}}(\pi \mathfrak{m}[\mathrm{v}, \mathrm{w}]) .
$$

Assim, através da identificação de $\mathfrak{m} \operatorname{com} T_{x_{0}} M$. o vetor $\xi^{\omega} \in \mathfrak{m}$ definido na proposição (3.8) corresponde ao vetor $\xi_{\omega} \in T_{x_{0}} M$ definido na proposição (3.1). isto é $\xi_{\omega}=\pi_{\star}\left(\xi^{\omega}\right)$. Portanto, da proposição (3.8), segue que o produto interno $g_{x_{0}}^{\theta^{\alpha}}$ definido em $\mathcal{D}_{x_{0}}$ que corresponde a declarar $\xi_{\theta^{\alpha}}$ ortonormais e perpendiculares a $\mathfrak{p}$ pode ser intrinsicamente definido por $\bar{B}$ :

$$
g_{x_{0}}^{\theta^{\alpha}}(\cdot, \cdot)=\bar{B}\left(\pi_{*}^{-1} \cdot, \pi_{*}^{-1} \cdot\right)
$$

qualquer que seja a base ortonormal $\left(\theta^{\alpha}\right)_{x_{0}}$ fixada em $T_{x_{0}} M$.

Logo, no ponto base o produto interno definido pela relação (3.6), no caso sub-simétrico é dado por:

$$
<\cdot, \cdot>_{x_{0}}=\int_{O(d)} g_{x_{0}}^{A \cdot\left(\theta^{\alpha}\right)_{x_{0}}}(\cdot, \cdot) d \mu(A)=\int_{O(d)} \bar{B}\left(\pi_{*}^{-1} \cdot, \pi_{*}^{-1} \cdot\right) d \mu(A)=\bar{B}\left(\pi_{*}^{-1} \cdot, \pi_{x}^{-1} \cdot\right)
$$

\subsection{Sub-torções no caso sub-simétrico}

A sub-ALIO $(\mathfrak{g}, s, \mathfrak{k}, B), \mathfrak{g}=[\mathfrak{p}, \mathfrak{p}] \oplus \mathfrak{p}$, em um ponto $x_{0}$ de um espaço sub-simétrico $M=$ $G / K^{\circ} \mathrm{com}$ distribuição fortemente geradora por colchetes determina as sub-torções $\tau_{x_{0}}$ e $\sigma_{x_{0}}$ :

Proposição 3.9 Sejam $\tau$ e $\sigma$ as sub-torções de $M ; v^{\prime}, w^{\prime} \in \mathcal{D}_{x_{0}} ; \xi^{\prime}, \eta^{\prime} \in \mathcal{D}_{x_{0}}{ }^{\perp}$. Sejam $\mathrm{v}, \mathrm{w}, \in \mathfrak{p} ; \xi, \eta \in \mathfrak{k}^{\perp}$ tais que $\pi_{*}(\mathrm{v})=\mathrm{v}^{\prime}, \pi_{*}(\mathrm{w})=\mathrm{w}^{\prime}, \pi_{*}(\xi)=\xi^{\prime}$ e $\pi_{*}(\eta)=\eta^{\prime}$. Então:

$$
\begin{aligned}
-2<\tau_{\xi^{\prime}}\left(\mathrm{v}^{\prime}\right), \mathrm{w}^{\prime}>_{x_{0}} & =B([\xi, \mathrm{v}], \mathrm{w})+B([\xi, \mathrm{w}], \mathrm{v}) \\
<\sigma_{\mathrm{v}^{\prime}}\left(\xi^{\prime}\right), \eta^{\prime}>_{x_{0}} & =0
\end{aligned}
$$

onde $\langle\cdot, \cdot\rangle$ é a métrica canônica associada à $M$.

\section{Prova:}


Seja s: $U \subset M \equiv G / K \rightarrow G$ uma seção local tal que $x_{0} \in U$. s $\left(x_{0}\right)=1$ e tal que a imagem $(d \mathrm{~s})_{x_{0}}$ seja $\mathrm{m}=\mathfrak{k}^{\perp} \oplus \mathfrak{p}$. Consideremos os campos vetoriais $\vec{V}, \vec{W}, \vec{\xi}, \vec{\eta}$ em $U$, associados respectivamente aos vetores $v, w . \xi . \eta$, como no lema (3.2). Observando que:

$$
\begin{aligned}
<\vec{V}, \vec{W}>_{x} & =\left\langle\left(d \bar{L}_{\mathrm{s}(x)}\right)_{x_{0}}\left(\pi_{*}(\mathrm{v})\right),\left(d \bar{L}_{\mathrm{s}(x)}\right)_{x_{0}}\left(\pi_{*}(\mathrm{w})\right)\right\rangle_{x} \\
& =\left\langle\pi_{*}(\mathrm{v}), \pi_{*}(\mathrm{w})>_{x_{0}}=\left\langle\mathrm{v}^{\prime}, \mathrm{w}^{\prime}\right\rangle_{x_{0}}\right. \\
<\vec{\xi}, \vec{\eta}>_{x} & =\left\langle\xi^{\prime}, \eta^{\prime}>_{x_{0}}\right.
\end{aligned}
$$

$\forall x \in U$, pois $\bar{L}_{\mathrm{s}(x)}$ é isometria; do Teorema (1.1) segue que

$$
\begin{aligned}
-2<\tau_{\vec{\xi}}(\vec{V}) \cdot \vec{W}> & =\left\langle[\vec{\xi}, \vec{V}]^{\top}, \vec{W}>+<[\vec{\xi}, \vec{W}]^{\top}, \vec{V}>\right. \\
2<\sigma_{\vec{V}}(\vec{\xi}), \vec{\eta}> & =\left\langle[\vec{V}, \vec{\xi}]^{\perp}, \vec{\eta}>+\left\langle[\vec{V}, \vec{\eta}]^{\perp}, \vec{\xi}\right\rangle\right.
\end{aligned}
$$

Assim, usando o lema (3.2), no ponto $x_{0}$ temos que:

$$
\begin{aligned}
-2<\tau_{\xi}\left(\mathrm{v}^{\prime}\right), \mathrm{w}^{\prime}>_{x_{0}} & =<\pi_{*}([\xi, \mathrm{v}]), \pi_{*}(\mathrm{w})>_{x_{0}}+<\pi_{*}([\xi, \mathrm{w}]), \pi_{*}(\mathrm{v})>_{x_{0}} \\
& =B([\xi, \mathrm{v}], \mathrm{w})+B([\xi, \mathrm{w}], \mathrm{v}) \\
<\sigma_{\mathrm{v}^{\prime}}\left(\xi^{\prime}\right), \eta^{\prime}>_{x_{0}} & =0
\end{aligned}
$$

uma vez que $\left[\mathfrak{k}^{\perp}, \mathfrak{p}\right] \subset[[\mathfrak{p}, \mathfrak{p}], \mathfrak{p}] \subset \mathfrak{p}$ e $\pi_{*}(\mathfrak{p})=\mathcal{D}_{x_{0}}$. 


\section{Capítulo 4}

\section{Espaços sub-simétricos de codimensão três com distribuição fortemente geradora por colchetes}

Se $(M, \mathcal{D}, g)$ é uma variedade sub-Riemanniana com distribuição fortemente geradora por colchetes de posto $n$ e codimensão $d \geq 2$, então $n$ é um múltiplo de 4 e $n \geq d+1$. Veja demonstração em [8].

Quando a codimensão é um, a condição fortemente geradora por colchetes é equivalente a condição de contato, neste caso os espaços sub-simétricos simplesmente conexos já estão completamente classificados segundo a sequência de artigos [1], [2], [3].

É natural então estudar esses espaços, sub-simétricos com distribuição fortemente geradora por colchetes, em codimensões mais altas. Neste capítulo apresentaremos a nossa contribuição nesta direção. No que segue, salvo menção explícita em contrário. sempre que nos referirmos a um espaço sub-simétrico subentende-se que este espaço é simplesmente conexo, com distribuição fortemente geradora por colchetes de codimensão três.

\subsection{Classificação dos espaços sub-simétricos em dimen- são sete com grupo de isometrias nilpotente}

Nesta seção, descrevemos a estrutura especial das LISO-álgebras associadas aos espaços subsimétricos, de dimensão sete, que possuem um grupo de Lie de isometrias sub-Riemannianas, nilpotente, agindo transitivamente. Mostramos que em particular, estes espaços são grupos de Lie. Os dois lemas seguintes nos serão úteis posteriormente.

Lema 4.1 Seja $(\mathfrak{g}, s, \mathfrak{k}, B)$ uma LISO-álgebra e seja Kill a forma de Killing de $\mathfrak{g}$. Se a e b são subespaços $K_{\text {ill }}$-ortogonais de $\mathfrak{p}$ tal que b é ad $d_{\mathfrak{k}}$-invariante, então $[a, b] \cap \mathfrak{k}=0$. 
Prova: Semelhante à prova do Lema 8.2.1 de [1i].

Lema 4.2 Se $(\mathfrak{g}, s, \mathfrak{k}, B)$ é uma LISO-álgebra tal que g é uma álgebra de Lie nilpotente então ela admite uma LISO-subálgebra $\left(\mathfrak{g}^{\prime}, s^{\prime}, \mathfrak{k}^{\prime}, B^{\prime}\right)$ com $\left[\mathfrak{p}^{\prime}, \mathfrak{p}^{\prime}\right]=\mathfrak{h}^{\prime}, \mathfrak{k}^{\prime}=0$ e $\mathfrak{g}^{\prime}$ nilpotente.

\section{Prova:}

Visto que $\mathfrak{g}$ é nilpotente, sua forma de Killing $K_{i l l}$ é nula. Em particular $K_{i l l}(\mathfrak{p}, \mathfrak{p})=0$. Pelo lema (4.1) aplicado à $a=b=\mathfrak{p}$, tem-se $[\mathfrak{p}, \mathfrak{p}] \cap \mathfrak{k}=0$. Assim, da proposição (3.6). segue que $(\mathfrak{g}, s, \mathfrak{k}, B)$ admite uma LISO-subálgebra com as propriedades requeridas.

A proposição abaixo é o resultado principal desta seção.

Proposição 4.1 Seja $(\mathfrak{g}, s, \mathfrak{k}, B)$ uma LISO-álgebra com $\mathfrak{k}=0, \mathfrak{h}=[\mathfrak{p}, \mathfrak{p}]$, g nilpotente, dim $\mathfrak{p}=4$ e dim $\mathfrak{g}=\imath$ então $\mathfrak{h}$ é abeliana $e[\mathfrak{h}, \mathfrak{p}]=0$.

\section{Prova:}

Visto que $\mathfrak{g}$ é nilpotente, $\mathfrak{g}=\mathfrak{h} \oplus \mathfrak{p},[\mathfrak{h}, \mathfrak{h}] \subset \mathfrak{h}$ e $[\mathfrak{h}, \mathfrak{p}] \subset \mathfrak{p}$, temos que $\left.a d_{h}\right|_{\mathfrak{p}}: \mathfrak{p} \rightarrow \mathfrak{p}$ é nilpotente $\forall h \in \mathfrak{h}$. Portanto, pelo Teorema de Engel ([6], Teorema 2.4), existe $x_{1} \neq 0$, $x_{1} \in \mathfrak{p}$ tal que $a_{h}\left(x_{1}\right)=0 \forall h \in \mathfrak{h}$. Como $\mathfrak{h}$ é uma subálgebra de Lie nilpotente, de dimensão três, temos apenas duas possibilidades para $\mathfrak{h}: \mathfrak{h}=\operatorname{Lie}\left(H^{3}\right)$ (álgebra de Lie do grupo de Heisenberg) ou $\mathfrak{h}$ abeliana. Mas, $\mathfrak{h}=\operatorname{Lie}\left(H^{3}\right)$ não pode ocorrer. De fato, suponhamos $\mathfrak{h}=\operatorname{Lie}\left(H^{3}\right)$ e tomemos $x_{1} \neq 0, x_{1} \in \mathfrak{p}$ dado pelo Teorema de Engel, como acima, entào da hipótese fortemente geradora por colchetes. existe $x_{2} \in \mathfrak{p}$ tal que $\left[x_{1}, x_{2}\right]=y$, onde $y$ é tal que $\mathfrak{z}\left(\operatorname{Lie}\left(H^{3}\right)\right)=\mathbb{R} y$ (a notação $\mathfrak{j}\left(\operatorname{Lie}\left(H^{3}\right)\right)$ indica o centro da álgebra de Lie do grupo de Heisenberg, que como sabemos, é 1-dimensional). Completamos para uma base $\left\{x_{1}, x_{2}, x_{3}, x_{4}\right\}$ de $\mathfrak{p}$, e novamente da hipótese fortemente geradora por colchetes, temos que $\left\{\left[x_{1}, x_{2}\right]=y,\left[x_{1}, x_{3}\right]=z,\left[x_{1}, x_{4}\right]=w\right\}$ é base de $\operatorname{Lie}\left(H^{3}\right)$, podemos ainda supor que $[z, w]=y$ (caso contrário renomeamos $x_{2}$, de tal maneira que esta suposição seja verificada). Agora, usando as identidades de Jacobi, temos: $\left[z, x_{4}\right]=\left[\left[x_{1}, x_{3}\right], x_{4}\right]=\left[\left[x_{1}, x_{4}\right], x_{3}\right]=\left[w, x_{3}\right]$; e segue a contradição: $y=[z, w]=\left[\left[x_{1}, x_{3}\right], w\right]=\left[\left[w, x_{3}\right], x_{1}\right]=\left[\left[z, x_{4}\right], x_{1}\right]=\left[\left[x_{1}, x_{4}\right], z\right]=$ $[w, z]=-y$. Assim, a única possibilidade é $\mathfrak{h}=[\mathfrak{p}, \mathfrak{p}]$ abeliana. Vamos provar agora, que no caso $\mathfrak{h}$ abeliana tem-se necessariamente $[\mathfrak{h}, \mathfrak{p}]=0$. Suponha que $\mathfrak{h}$ é abeliana e $[\mathfrak{h}, \mathfrak{p}] \neq 0$, então existe $z \neq 0 \in \mathfrak{h}$ e $u \neq 0 \in \mathfrak{p}$ tal que $[z, u] \neq 0$. Agora, $\left.a d_{z}\right|_{\mathfrak{p}}: \mathfrak{p} \rightarrow \mathfrak{p}$ é nilpotente, portanto a dimensão do ker $\left.a d_{z}\right|_{\mathfrak{p}}$ é maior ou igual a um, assim é sempre possível escolher dois elementos, $u_{1}$ e $\left[z, u_{2}\right]$ linearmente independentes em $\mathfrak{p}, u_{1} \in\left(\left.\operatorname{ker} a d_{z}\right|_{\mathfrak{p}}\right)$ e $\left[z, u_{2}\right] \in a d_{z}(\mathfrak{p})$ que tomados desta maneira satisfazem $\left[u_{1},\left[z, u_{2}\right]\right]=0$, uma vez que $[\mathfrak{h}, \mathfrak{h}]=0$ Mas, este 
fato é uma contradição. pois o comutador de dois elementos linearmente independentes de $\mathfrak{p}$ tem que ser diferente de zero, já que $\theta_{u}: \mathfrak{p} \rightarrow[\mathfrak{p}, \mathfrak{p}]$ é sobrejetora $\forall u \in \mathfrak{p}$, $\operatorname{dim} \mathfrak{p}=4 \mathrm{e}$ $\operatorname{dim}[\mathfrak{p}, \mathfrak{p}]=3$. Portanto. $\mathfrak{h}$ é abeliana e $[\mathfrak{h}, \mathfrak{p}]=0$.

Podemos reenunciar a proposição (4.1) da seguinte maneira.

Teorema 4.1 Seja (p. B) um espaço vetoria real 4-dimensional com produto interno. hu um espaço vetorial real de dimensão três $e[\cdot, \cdot]: \mathfrak{p} \times \mathfrak{p} \rightarrow \mathfrak{h}$ uma aplicação bilinear anti-simétrica tal que $i_{X} \circ[\cdot, \cdot]: \mathfrak{p} \rightarrow \mathfrak{h}$ é sobrejetora $\forall X \neq 0 \in \mathfrak{p}$. Então, $(\mathfrak{g}=\mathfrak{p} \oplus \mathfrak{h}, s, \mathfrak{k}=0, B)$ é uma LISO-álgebra nilpotente. onde o colchete de Lie em $\mathfrak{g}$ é a extensão da aplicação bilinear $[\cdot \cdot]$ dada por $[\mathfrak{p}, \mathfrak{h}]=0,[\mathfrak{h} . \mathfrak{h}]=0$ e a involução $s: \mathfrak{g} \rightarrow \mathfrak{g} e ́$ definida por $s(X)=-X, \forall X \in \mathfrak{p} e$ $s(X)=X, \forall X \in \mathfrak{h}$. Além disso, toda LISO-álgebra $(\mathfrak{g}=\mathfrak{p} \oplus \mathfrak{h}, s, \mathfrak{k}=0, B)$ nilpotente com $\mathfrak{h}=[\mathfrak{p}, \mathfrak{p}], \operatorname{dim} \mathfrak{g}=i \in \operatorname{dim} \mathfrak{p}=4$ é desse tipo.

Concluimos que se $(K, \mathcal{D}, g)$ é um espaço sub-simétrico de dimensão 7 , simplesmente conexo, munido de distribuição fortemente geradora por colchetes de posto 4, com um grupo de Lie nilpotente transitivo de isometrias sub-Riemannianas de $M$, então $M$ admite uma LISO-álgebra $(\mathfrak{g}, s, \mathfrak{k}, B) \operatorname{com} \mathfrak{g}=[\mathfrak{p}, \mathfrak{p}] \oplus \mathfrak{p}$ nilpotente, $\operatorname{dim} \mathfrak{g}=7, \operatorname{dim} \mathfrak{p}=4, \mathfrak{k}=0 . \mathfrak{h}=$ $[\mathfrak{p}, \mathfrak{p}]$ abeliana e $[\mathfrak{h}, \mathfrak{p}]=0$; em particular $M$ é um grupo de Lie. Neste caso, existe $3=$ $\left\{x_{1}, x_{2}, x_{3}, x_{4}, y, z, w\right\}$ base de $\mathfrak{g}$, onde $\left\{x_{1}, x_{2}, x_{3}, x_{4}\right\}$ é base ortonormal de $\mathfrak{p}$, satisfazendo:

$$
\begin{array}{ll}
{\left[x_{1}, x_{2}\right]=y} & a_{i j}, b_{i j} \in \mathbb{R} \\
{\left[x_{1}, x_{3}\right]=z} & \quad(i, j=2,3,4 ; i<j) \\
{\left[x_{1}, x_{4}\right]=w} & \text { com } \\
{\left[x_{2}, x_{3}\right]=a_{23} y+b_{23} z+c_{23} w} \\
{\left[x_{2}, x_{4}\right]=a_{24} y+b_{24} z+c_{24} w} \\
{\left[x_{3}, x_{4}\right]=a_{34} y+b_{34} z+c_{34} w}
\end{array} \quad\left\{\begin{array}{l}
b_{23} c_{24}-c_{23} b_{24} \neq 0 \\
c_{23} a_{34}-a_{23} c_{34} \neq 0 \\
a_{24} b_{34}-b_{24} a_{34} \neq 0
\end{array}\right.
$$

Uma classe especial desses exemplos nilpotentes, que denominaremos de exemplos de tipo diagonal, é dada quando: $a_{23}=b_{23}=a_{24}=c_{24}=b_{34}=c_{34}=0$. Neste caso. 
renomeando as constantes de estrutura (para simplificar a notaçào). temos que existe $3=$ $\left\{x_{1}, x_{2}, x_{3}, x_{4}, y, z, w\right\}$ base ortonormal de $\mathfrak{g}$, onde $\left\{x_{1}, x_{2}, x_{3}, x_{4}\right\}$ é base ortonormal de $\mathfrak{p}$, satisfazendo:

$$
\begin{aligned}
& {\left[x_{1}, x_{2}\right]=\frac{1}{\sqrt{1+a^{2}}} y} \\
& {\left[x_{1}, x_{3}\right]=\frac{1}{\sqrt{1+b^{2}}} z} \\
& {\left[x_{1}, x_{4}\right]=\frac{1}{\sqrt{1+c^{2}}} w} \\
& {\left[x_{2}, x_{3}\right]=\frac{c}{\sqrt{1+c^{2}}} w} \\
& {\left[x_{2}, x_{4}\right]=\frac{b}{\sqrt{1+b^{2}}} z} \\
& {\left[x_{3}, x_{4}\right]=\frac{a}{\sqrt{1+a^{2}}} y}
\end{aligned}
$$

e os demais colchetes são nulos

Entào denotando por $\left\{\theta^{1}, \theta^{2}, \theta^{3}, \theta^{4}, \theta^{y}, \theta^{z}, \theta^{w}\right\}$ a base dual de $\left\{x_{1}, x_{2}, x_{3}, x_{4}, y, z, w\right\}$ e dualizando as equações acima, temos as equações de estrutura:

$$
\begin{aligned}
d \theta^{i}= & i=1,2,3,4 \\
d \theta^{y}= & -\frac{1}{\sqrt{1+a^{2}}} \theta^{1} \wedge \theta^{2}-\frac{a}{\sqrt{1+a^{2}}} \theta^{3} \wedge \theta^{4} \\
d \theta^{z}= & -\frac{1}{\sqrt{1+b^{2}}} \theta^{1} \wedge \theta^{3}-\frac{b}{\sqrt{1+b^{2}}} \theta^{2} \wedge \theta^{4} \\
d \theta^{w} & =-\frac{1}{\sqrt{1+c^{2}}} \theta^{1} \wedge \theta^{4}-\frac{c}{\sqrt{1+c^{2}}} \theta^{2} \wedge \theta^{3}
\end{aligned}
$$

De onde obtemos que, nos exemplos de tipo diagonal, as formas de conexão $\omega_{j}^{i}$ e $\omega_{, 3}^{\alpha}$ são todas nulas, as sub-torções $\tau$ e $\sigma$ também são ambas nulas, restando apenas os invariantes $\rho_{i}(i=1, \ldots, 4)$ relativos ao operador bilinear $A: \mathcal{D}^{\perp} \times \mathcal{D}^{\perp} \rightarrow \operatorname{End}(\mathcal{D})$, definidos no capítulo 1 , seção 1.3 , dados por: 


$$
\begin{aligned}
& \rho_{1}=-\frac{1}{1+a^{2}}-\frac{1}{1+b^{2}}-\frac{1}{1+c^{2}}, \\
& \rho_{2}=-\frac{1}{1+a^{2}}-\frac{b^{2}}{1+b^{2}}-\frac{c^{2}}{1+c^{2}}, \\
& \rho_{3}=-\frac{a^{2}}{1+a^{2}}-\frac{1}{1+b^{2}}-\frac{c^{2}}{1+c^{2}}, \\
& \rho_{4}=-\frac{a^{2}}{1+a^{2}}-\frac{b^{2}}{1+b^{2}}-\frac{1}{1+c^{2}} ;
\end{aligned}
$$

os demais invariantes, definidos na seção 1.3, nos exemplos de tipo diagonal são nulos, exceto $\lambda_{\alpha}=-2(\alpha=5,6,7)$.

\subsection{Exemplos quaterniônicos}

De acordo com o Teorema (5.1) de [2], todo espaço sub-simétrico de contato, simplesmente conexo, irredutível é um espaço homogêneo canônicamente fibrado sobre um espaço simétrico Hermitiano irredutível, com fibras difeomorfas ao círculo $S^{1}$. Inspirados nesta idéia, construimos exemplos de espaços sub-simétricos munidos de distribuição fortemente geradora por colchetes, os quais correspondem a uma Sp(1)-fibração sobre espaços simétricos Riemannianos, compactos, simplesmente conexos. equipados com estrutura quaterniônica. O objetivo desta seção é expor a construção destes exemplos. Iniciamos introduzindo alguns resultados preliminares acerca de espaços simétricos Riemannianos com estrutura quaterniônica. Uma referência sobre este assunto pode ser encontrada em [16].

Seja $V$ um espaço vetorial real. Uma álgebra quaterniônica sobre $V$ é uma álgebra $\mathcal{A}$ de transformações lineares de $V$ isomorfa a álgebra real dos quatérnios, e cujo elemento unidade é a transformação identidade de $V$. Se $V$ é espaço com produto interno e os elementos unimodulares de $\mathcal{A}$ são transformações lineares ortogonais de $V$, diremos que $\mathcal{A}$ é uma álgebra quaterniônica ortogonal sobre $V$.

É interessante notar que se $\mathcal{A}$ é uma álgebra quaterniônica sobre $V$, então podemos visualizar $V$ como um espaço vetorial quaterniônico, basta considerar $V$ como espaço vetorial sobre $\mathcal{A}$. Sob esta ótica, as transformações lineares-quaterniônicas são justamente as transformações lineares que comutam com todo elemento de $\mathcal{A}$.

Seja $S$ uma variedade Riemanniana. Dado $x \in S$, denotamos por $\Psi_{x}$ o grupo de holonomia linear da conexão Riemanniana com referência no ponto $x$ (ie, $\Psi_{x}$ é o grupo consistindo 
de todas as transformaçòes lineares do espaço tangente $T_{x} S$ obtidas de transporte paralelo sobre curvas ligando $x$ a $x$ ). Um conjunto $A_{x}$ de transformações lineares de $T_{x} S$ é denominado $\Psi_{x}$-invariante se $g A_{x} g^{-1}=A_{x}, \forall g \in \Psi_{x}$. Um conjunto $A$ de campos de transformaçòes lineares de todos os espaços tangentes de $S$ é denominado paralelo se, dado $x, y \in S$ e uma curva $\sigma$ ligando $x$ a $y$, o transporte paralelo $\tau_{\sigma}$ ao longo de $\sigma$ satisfaz $\tau_{\sigma} A_{x} \tau_{\sigma}^{-1}=A_{y}$. $A_{x}$ se estende a um conjunto paralelo de campos de transformações lineares de espaços tangentes se, e somente se. $A_{x}$ é $\Psi_{x}$-invariante; neste caso a extensão é única e bem definida na notação acima por $A_{y}=\tau_{\sigma} A_{x} \tau_{\sigma}^{-1}$.

Uma estrutura quaternionica sobre uma variedade Riemanniana $S$ é um campo paralelo $A$ de álgebras quaterniônicas ortogonais $\mathcal{A}_{x}$ sobre cada espaço tangente $T_{x} S$.

Seja $S$ uma variedade Riemanniana com estrutura quaterniônica $A$, então dado $x \in S$, $\mathcal{A}_{x}$ é uma $\Psi_{x}$-estável álgebra quaterniônica ortogonal sobre $T_{x} S$. Em outras palaviras. $\Psi_{x}=$ $\Phi_{x} \cdot \mathcal{A}_{x}^{\prime}$ onde $\Phi_{x}$ é o centralizador de $\mathcal{A}_{x}$ em $\Psi_{x}$ e $\mathcal{A}_{x}^{\prime}=\Psi_{x} \cap \mathcal{A}_{x}$. $\Phi_{x}$ e $\mathcal{A}_{x}^{\prime}$ são, respectivamente as partes A-linear e A-escalar de $\Psi_{x}$. Dizemos que o grupo de holonomia possui parte $\epsilon$ scalar real se os elementos de $\mathcal{A}_{x}^{\prime}$ sào escalares reais (1 ou -1), possui parte escalar complera se $\mathcal{A}_{x}^{\prime}$ está contida em um subcorpo complexo de $\mathcal{A}_{x}$ que não é um subcorpo real, possui parte escalar quaterniônica caso contrário (neste caso $\mathcal{A}_{x}^{\prime}$ gera $\mathcal{A}_{x}$ ).

Estamos particularmente interessados nos espaços simétricos Riemannianos com estrutura quaterniônica na qual a holonomia tem parte escalar quaterniônica, por simplicidade denominaremos estes espaços de espaços de Wolf quaternionicos. Tal interesse reside no fato que nossos exemplos são construidos através desta classe de espaços.

Antes, é conveniente lembrar que, uma álgebra de lie involutiva ortogonal (ALIO) é uma trinca $(\mathfrak{g}, s, B)$. onde $\mathfrak{g}$ é uma álgebra de Lie real, $s$ é um automorfismo involutivo de $\mathfrak{g}, o$ auto-espaço +1 de $s$ (denotado por $\mathfrak{h}$ ) não contém ideais não nulos de $\mathfrak{g}$ e $B$ é um produto interno em $\mathfrak{p}$ (auto-espaço -1 de $s$ ) invariante pela representação adjunta de $\mathfrak{h}$.

Uma ALIO $(\mathfrak{g}, s, B)$ é irredutivel se $[\mathfrak{p}, \mathfrak{p}] \neq 0$ e $a d_{\mathfrak{h}}$ é irredutivel sobre $\mathfrak{p}$.

Expomos agora alguns resultados referentes aos espaços de Wolf quaterniônicos. Maiores detalhes podem ser encontrados em [16].

Da caracterização dos espaços simétricos quaterniônicos temos, segundo ([16], Teorema 5.4) e sua demonstração, que existe uma correspondência biunívoca entre os espaços simétricos Riemannianos, compactos, simplesmente conexos, com estrutura quaterniônica na qual a holonomia tem parte escalar quaterniônica e as ALIO's $\left(\mathfrak{g}_{u}, s, B\right)$ com as propriedades adicionais:

i.) $\mathfrak{g}_{u}$ é compacta e simples, em particular $\left(\mathfrak{g}_{u}, s, B\right)$ é irredutivel e consequentemente 
existe um único $a \in \mathbb{R}$. $a<0$ tal que $B=\left.a K_{i l l}\right|_{\mathfrak{p} \times \mathfrak{p}}$. onde $K_{i l l}$ denota a forma de Killing de $\mathfrak{g}_{u}$ e $\mathfrak{p}$ denota o auto-espaço -1 de $s$;

ii.) a involução $s$ e os auto-espaços \pm 1 de $s$, são caracterizados da seguinte maneira: escolhe-se $\mathfrak{I} \subset\left(\mathfrak{g}_{u}\right)$ a álgebra de Lie de um toro máximo. Denota-se por $\mathfrak{G}$ e $\mathfrak{H}$ as respectivas complexificações de $\mathfrak{g}_{u}$ e $\mathfrak{T}$. Tem-se que $\mathfrak{H}$ é uma subálgebra de Cartan da álgebra de Lie simples complexa $\mathfrak{G}$ e toma-se a decomposição de Cartan

$$
\mathfrak{G}=\mathfrak{H} \oplus \sum_{\lambda \in \Delta} \mathfrak{H}_{\lambda}
$$

onde $\Delta$ é o conjunto de todas as raízes de $\mathfrak{G}$ relativas à $\mathfrak{H}$. e $\mathfrak{G}_{\lambda}$ é o subespaço 1-dimensional complexo definido por:

$$
\mathfrak{G}_{\lambda}=\left\{X \in \mathfrak{G}: \operatorname{ad}_{H}\left(X^{\prime}\right)=\lambda(H) X \quad \forall H \in \mathfrak{H}\right\}
$$

Dados $\lambda, \rho \in \Delta$. indica-se por $H_{\lambda}$ o elemento de $\mathfrak{H}$ caracterizado por: $K_{\text {ill }}\left(H_{\lambda}, H\right)=$ $\lambda(H) \forall H \in \mathfrak{H}$, e abrevia-se $K_{i l l}\left(H_{\lambda}, H_{\beta}\right)$ por $K_{i l l}(\lambda . \beta)$. Escolhe-se uma ordem lexicográfica sobre as raízes e denota-se por $\rho$ a raiz máxima relativa a esta ordem. Então $s=A d_{\psi}: \mathfrak{g} \rightarrow \mathfrak{g}$ onde $\psi=\exp \left(\pi \frac{i}{c} H_{\rho}\right), c=K_{i l l}(\rho, \rho) / 2$. O auto-espaço +1 de $s$, denotado por $\mathfrak{h}$, é tal que: $\mathfrak{h}=\mathfrak{l}_{1} \oplus \mathfrak{a}_{1}$, é uma soma direta de ideais onde

$$
\begin{aligned}
& \mathfrak{l}_{1}=\{H \in \mathfrak{T}: \rho(H)=0\}+ \sum_{\lambda>0} \mathfrak{g}_{u} \cap\left(\mathfrak{G}_{\lambda}+\mathfrak{G}_{-\lambda}\right) \\
& \kappa_{i l l}^{-}(\lambda, \rho)=0 \\
& \mathfrak{a}_{1}=\mathfrak{g}_{u} \cap\left(\mathbb{C} H_{\rho}+\mathfrak{G}_{\rho}+\mathfrak{H}_{-\rho}\right) \cong \mathfrak{s p}(1)
\end{aligned}
$$

O auto espaço -1 de $s$, denotado por $\mathfrak{p}$, é dado por:

$$
\mathfrak{p}=\sum_{\substack{\rho \neq \lambda>0 \\ K_{i l l}(\lambda, \rho) \neq 0}} \mathfrak{g}_{u} \cap\left(\mathfrak{G}_{\lambda}+\mathfrak{G}_{-\lambda}\right) .
$$

Esta particularidade da ALIO associada a um espaço de Wolf quaterniônico, simplesmente conexo, compacto, por consequência irredutível. (denominada ALIO quaterniônica compacta irredutivel) nos permite construir a partir deste, de maneira bastante natural, um espaço 
sub-simétrico, compacto. simplesmente conexo. com distribuiçào fortemente geradora por colchetes de codimensão três. O corolário da proposiçào [4.1] nos conta como é feita tal construção, antes porém faz se necessário os seguintes resultados preliminares:

Definição 4.1 Uma base quaterniônica de uma álgebra quaterniônica $\mathcal{A}$ é uma base $\beta=$ $\left\{I d, J_{1}, J_{2}, J_{3}\right\}$ de $\mathcal{A}$ tal que: $J_{i} \circ J_{i}=-I d(i=1,2,3) ; J_{i} \circ J_{k}=-J_{k} \circ J_{i}($ para $i \neq k)$; $J_{1} \circ J_{2}=J_{3}, J_{2} \circ J_{3}=J_{1} . J_{3} \circ J_{1}=J_{2}$.

Lema 4.3 Seja $\left(\mathfrak{g}_{u}, s, B\right)$ uma ALIO quaternionica compacta irredutível, consideremos a decomposição $\mathfrak{g}_{u}=\mathfrak{h} \oplus \mathfrak{p}$ sobs, $\mathfrak{h}=\mathfrak{l}_{1} \oplus \mathfrak{a}_{1} \operatorname{com} \mathfrak{l}_{1}, \mathfrak{a}_{1}$ e $\mathfrak{p}$ dados por (4.1), (4.2) e (4.3). respectivamente. Nesta notação, sejam $E_{\rho} \in \mathfrak{G}_{\rho}$ e $E_{-\rho} \in \mathfrak{G}_{-\rho}$ tais que $K_{\text {ill }}\left(E_{\rho}, E_{-\rho}\right)=1$. Então :

a. $\gamma=\left\{\xi_{1}=\frac{1}{c} i H_{\rho}, \xi_{2}=\frac{1}{\sqrt{c}}\left(E_{\rho}-E_{-\rho}\right), \xi_{3}=\frac{1}{\sqrt{c}} i\left(E_{\rho}+E_{-\rho}\right)\right\}$ é base $a_{1}$, satisfazendo $\left[\xi_{1}, \xi_{2}\right]=2 \xi_{3},\left[\xi_{1}, \xi_{3}\right]=-2 \xi_{2},\left[\xi_{2}, \xi_{3}\right]=2 \xi_{1}$;

b. $\beta=\left\{I d, J_{1}, J_{2}, J_{3}\right\}$, onde $J_{i}=\left.\operatorname{Ad}\left(\exp \frac{\pi}{2} \xi_{i}\right)\right|_{\mathfrak{p}}=\left.\operatorname{ad}_{\xi_{i}}\right|_{\mathfrak{p}}(i=1,2,3)$ gera uma álgebra quaterniônica ortogonal sobre $\mathfrak{p}$ com $\beta$ base quaterniônica.

\section{Prova:}

a. É consequência das seguintes identidades:

$$
\begin{array}{ll}
{\left[H_{\rho}, E_{\rho}\right]=K_{i l l}(\rho, \rho) E_{\rho}=2 c E_{\rho}} & {\left[H_{\rho}, E_{-\rho}\right]=-K_{i l l}(\rho, \rho) E_{-\rho}=-2 c E_{-\rho}} \\
{\left[E_{\rho}, E_{-\rho}\right]=K_{i l l}\left(E_{\rho}, E_{-\rho}\right) H_{\rho}=H_{\rho}} & {\left[E_{\rho}, E_{\rho}\right]=\left[E_{-\rho}, E_{-\rho}\right]=0}
\end{array}
$$

pois $2 \rho$ não é uma raiz.

b. Temos as seguintes identidades:

$$
\left[H_{\rho}, E_{\lambda}\right]=K_{i l l}(\rho, \lambda) E_{\lambda} \quad\left[H_{\rho}, E_{-\lambda}\right]=-K_{i l l}(\rho, \lambda) E_{-\lambda}
$$

Se $\lambda$ é raiz positiva, também temos:

$$
\begin{array}{r}
{\left[E_{\rho}, E_{\lambda}\right]=\left[E_{-\rho}, E_{-\lambda}\right]=0 \quad\left[E_{\rho},\left[E_{\rho}, E_{-\lambda}\right]\right]=\left[E_{-\rho},\left[E_{-\rho}, E_{\lambda}\right]\right]=0} \\
\text { pois. } \rho+\lambda \text { e } 2 \rho-\lambda \text { não são raízes. }
\end{array}
$$

Como $\rho$ é raiz máxima, vale que $2 K_{i l l}(\rho, \lambda) / K_{i l l}(\rho, \rho)=0$ ou 1 para toda raiz positiva $\lambda$, veja demonstração em ([16], seção 4). Portanto, se $\lambda$ é raiz positiva tal que $K_{i l l}(\rho, \lambda) \neq 0$ então, 
$K_{i l l}(\rho, \lambda)=K_{i l l}(\rho, \rho) / 2=c$. Segundo a relação (4.3), um sistema de geradores de $\mathfrak{p}$ é dado por $S=\left\{E_{\lambda}-E_{-\lambda}, i\left(E_{\lambda}+E_{-\lambda}\right): \lambda \neq \rho\right.$ raiz positiva $\left.e K_{i l l}(\rho, \lambda) \neq 0\right\}$. Assim, respeitando as considerações acima e utilizando as identidades de Jacobi, um cálculo direto nos elementos de $S$, mostra que $\left.\left(a d_{\xi_{i}} \circ a d_{\xi_{i}}\right)\right|_{\mathfrak{p}}=-\left.I d\right|_{\mathfrak{p}}$ e portanto $J_{i}=\left.\operatorname{Ad}\left(\exp \frac{\pi}{2} \xi_{i}\right)\right|_{\mathfrak{p}}=\left.\exp \left(a d \frac{\pi}{2} \xi_{i}\right)\right|_{\mathfrak{p}}=$ $\left.\exp \left(\frac{\pi}{2} a d \xi_{i}\right)\right|_{\mathfrak{p}}=\left.a d_{\xi_{i}}\right|_{\mathfrak{p}}, i=1,2.3$; mostra também que os elementos de $\beta$ satisfazem, de fato, as relaçòes quaterniônicas dadas na definicão (4.2). Finalmente, a $B$-ortogonalidade das transformações $J_{i}(\mathrm{i}=1,2,3)$, decorre da $A d_{\mathfrak{h}}$-invariância do produto interno $B$.

Lema 4.4 Se $V$ é um espaço vetorial real com produto interno de dimensão $4 n$ e $\beta=$ $\left\{I d, J_{1}, J_{2}, J_{3}\right\}$ gera uma álgebra quaterniônica ortogonal sobre $V$, com $\beta$ base quaterniônica, então existe $\left\{X_{1}, J_{1}\left(X_{1}\right), J_{2}\left(X_{1}\right), J_{3}\left(X_{1}\right), \ldots, X_{n}, J_{1}\left(X_{n}\right), J_{2}\left(X_{n}\right), J_{3}\left(X_{n}\right)\right\}$ base ortonormal de $V$.

Prova: Faremos a demonstraçào usando indução em $n$.

Se $n=1$, seja $X_{1}$ unitário em $V$ e tem-se $\left\{X_{1}, J_{1}\left(X_{1}\right), J_{2}\left(X_{1}\right), J_{3}\left(X_{1}\right)\right\}$ linearmente independe contido em $V$. De fato, suponhamos que existam escalares $a_{1}, a_{2}, a_{3}, a_{4} \in \mathbb{R}$ tais que $a_{1} X_{1}+a_{2} J_{1}\left(X_{1}\right)+a_{3} J_{2}\left(X_{1}\right)+a_{4} J_{3}\left(X_{1}\right)=0$ então, usando que $J_{1}, J_{2}, J_{3}$ são endomorfismos de $V$ cujo quadrado é igual a -1 , que anti-comutam, e tais que $J_{1} \circ J_{2}=J_{3}, J_{2} \circ J_{3}=J_{1}$, $J_{3} \circ J_{1}=J_{2}$, segue que:

$$
\left\{\begin{array}{l}
a_{1} X_{1}+a_{2} J_{1}\left(X_{1}\right)+a_{3} J_{2}\left(X_{1}\right)+a_{4} J_{3}\left(X_{1}\right)=0 \\
a_{1} J_{1}\left(X_{1}\right)-a_{2} X_{1}+a_{3} J_{3}\left(X_{1}\right)-a_{4} J_{2}\left(X_{1}\right)=0 \\
a_{1} J_{2}\left(X_{1}\right)-a_{2} J_{3}\left(X_{1}\right)-a_{3} X_{1}+a_{4} J_{1}\left(X_{1}\right)=0 \\
a_{1} J_{3}\left(X_{1}\right)+a_{2} J_{2}\left(X_{1}\right)-a_{3} J_{1}\left(X_{1}\right)-a_{4} X_{1}=0
\end{array}\right.
$$

$\mathrm{Ou}$, equivalentemente, em notação matricial:

$$
A \cdot\left[\begin{array}{c}
X_{1} \\
J_{1}\left(X_{1}\right) \\
J_{2}\left(X_{1}\right) \\
J_{3}\left(X_{1}\right)
\end{array}\right]=\left[\begin{array}{l}
0 \\
0 \\
0 \\
0
\end{array}\right] \text { onde } A=\left[\begin{array}{cccc}
a_{1} & a_{2} & a_{3} & a_{4} \\
-a_{2} & a_{1} & -a_{4} & a_{3} \\
-a_{3} & a_{4} & a_{1} & -a_{2} \\
-a_{4} & -a_{3} & a_{2} & a_{1}
\end{array}\right]
$$

Como $X_{1}, J_{1}\left(X_{1}\right), J_{2}\left(X_{1}\right), J_{3}\left(X_{1}\right)$ são todos não nulos, segue que $\operatorname{det}(A)=0$. Agora, $\operatorname{det}(A)=0$ se, e somente se, $a_{i}=0$ para $i=1,2,3,4$. 
Além disso, como $J_{1}, J_{2}, J_{3}$ são transformações lineares ortogonais temos:

$$
\begin{aligned}
<J_{1}\left(X_{1}\right), J_{1}\left(X_{1}\right)> & =<J_{2}\left(X_{1}\right), J_{2}\left(X_{1}\right)>=<J_{3}\left(X_{1}\right), J_{3}\left(X_{1}\right)> \\
& =<X_{1}, X_{1}>=1 \\
<X_{1}, J_{2}\left(X_{1}\right)> & =<J_{2}\left(X_{1}\right),\left(J_{2} \circ J_{2}\right)\left(X_{1}\right)=-<X_{1}, J_{2}\left(X_{1}\right)> \\
& \Longrightarrow<X_{1}, J_{2}\left(X_{1}\right)>=0
\end{aligned}
$$

$$
\begin{aligned}
& \text { Analogamente, }\left\langle X_{1}, J_{3}\left(X_{1}\right)\right\rangle=\left\langle X_{1}, J_{1}\left(X_{1}\right)\right\rangle=0 \\
& \left\langle J_{1}\left(X_{1}\right), J_{2}\left(X_{1}\right)>=\left\langle\left(J_{3} \circ J_{1}\right)\left(X_{1}\right),\left(J_{3} \circ J_{2}\right)\left(X_{1}\right)\right\rangle=-<J_{2}\left(X_{1}\right), J_{1}\left(X_{1}\right)\right\rangle \\
& \Longrightarrow<J_{1}\left(X_{1}\right), J_{2}\left(X_{1}\right)>=0 \\
& \left\langle J_{1}\left(X_{1}\right), J_{3}\left(X_{1}\right)\right\rangle=\left\langle\left(J_{2} \circ J_{1}\right)\left(X_{1}\right),\left(J_{2} \circ J_{3}\right)\left(X_{1}\right)\right\rangle=-\left\langle J_{3}\left(X_{1}\right), J_{1}\left(X_{1}\right)\right\rangle \\
& \Longrightarrow<J_{1}\left(X_{1}\right), J_{3}\left(X_{1}\right)>=0 \\
& \left.\left\langle J_{2}\left(X_{1}\right), J_{3}\left(X_{1}\right)\right\rangle=\left\langle\left(J_{1} \circ J_{2}\right)\left(X_{1}\right),\left(J_{1} \circ J_{3}\right)\left(X_{1}\right)\right\rangle=-<J_{3}\left(X_{1}\right), J_{2}\left(X_{1}\right)\right\rangle \\
& \Longrightarrow<J_{2}\left(X_{1}\right), J_{3}\left(X_{1}\right)>=0
\end{aligned}
$$

Portanto $\left\{X_{1}, J_{1}\left(X_{1}\right), J_{2}\left(X_{1}\right), J_{3}\left(X_{1}\right)\right\}$ é base ortonormal de $V$.

- Hipótese de indução: suponha a tese válida para qualquer espaço vetorial de dimensão $4(n-1)$.

Vamos agora provar que o resultado também é verdade para os espaços vetoriais de dimensão $\operatorname{tn}(n>1)$. De fato, se $\operatorname{dim} V=4 n$, considere $X_{1} \in V$ unitário e do caso $n=1$ temos que $\gamma_{1}=\left\{X_{1}, J_{1}\left(X_{1}\right), J_{2}\left(X_{1}\right), J_{3}\left(X_{1}\right)\right\}$ é um conjunto ortonormal contido em $V$. Consideremos o subespaço 4-dimensional $W$ de $V$ gerado por $\gamma_{1}$ e seja $W^{\perp}$ o complemento ortogonal de $W$ em $V$. Observe que $W^{\perp}$ é $J_{1}, J_{2}, J_{3}$-invariante e portanto $\alpha=\left\{\left.I d\right|_{W_{\perp}^{\perp}},\left.J_{1}\right|_{W^{\perp}},\left.J_{2}\right|_{W^{\perp}},\left.J_{3}\right|_{W^{\perp}}\right\}$ gera uma álgebra quaterniônica ortogonal sobre $W^{\perp}$, com $\alpha$ uma base quaterniônica. Usando então a hipótese de induçào a $W^{\perp}$ temos que existe $\gamma_{2}=\left\{X_{2} . J_{1}\left(X_{2}\right), J_{2}\left(X_{2}\right), J_{3}\left(X_{2}\right), \ldots, X_{n}, J_{1}\left(X_{n}\right), J_{2}\left(X_{n}\right), J_{3}\left(X_{n}\right)\right\}$ uma base ortonormal de $W^{\perp}$. Portanto $\gamma=\gamma_{1} \cup \gamma_{2}$ é uma base ortonormal de $V$ satisfazendo as condições exigidas.

Definição 4.2 Uma LISO-álgebra $(\mathfrak{g}, s, \mathfrak{k}, B)$ é irredutivel se $[\mathfrak{p}, \mathfrak{p}] \cap \mathfrak{k} \neq 0$ e ad $\mathfrak{h}$ é irredutível sobre $\mathfrak{p}$. L'm espaço sub-simétrico, simplesmente conexo, $M=G / K$ é irredutícel se sua LISO-álgebra $(\mathfrak{g}, s, \mathfrak{k}, B)$ é irredutivel. 
Proposição 4.2 Seja $\left(\mathfrak{g}_{u}, s, B\right)$ uma ALIO quaterniônica compacta irredutivel e escreva $\mathfrak{g}_{u}=\mathfrak{h} \oplus \mathfrak{p} \operatorname{sob} s, \mathfrak{h}=\mathfrak{l}_{1} \oplus \mathfrak{a}_{1} \operatorname{com} \mathfrak{a}_{1} \cong \mathfrak{s p}(1)$. Então $\left(\mathfrak{g}_{u}, s, \mathfrak{l}_{1}, B\right)$ é uma LISO-ágtbra irredutivel.

\section{Prova:}

Seja $\gamma=\left\{\xi_{1}, \xi_{2}, \xi_{3}\right\}$ uma base de $\mathfrak{a}_{1}$ tal que $\left[\xi_{1}, \xi_{2}\right]=2 \xi_{3} \cdot\left[\xi_{1}, \xi_{3}\right]=-2 \xi_{2}$ e $\left[\xi_{2}, \xi_{3}\right]=2 \xi_{1}$, dada pelo Lema[4.3]. Seja $K_{i l l}$ a forma de Cartan Killing de $\mathfrak{g}_{u}$. então $\left.\mathfrak{g}_{u}=\mathfrak{l}_{1} \oplus<\xi_{1}, \xi_{2}, \xi_{3}\right\rangle \ni \mathfrak{p}$ é uma decomposição $K_{\text {ill }}$-ortogonal e além disso. pela irredutibilidade de $\left.a d_{\mathfrak{h}}\right|_{\mathfrak{p}}$, $\Lambda_{i l l}=$ $a B$ para algum escalar $a \neq 0$. Consideremos a álgebra quaterniônica ortogonal sobre $\mathfrak{p}$ gerada pela base quaterniônica $\left\{I d, J_{1}, J_{2}, J_{3}\right\}$ onde $J_{i}=.\left.t d\left(\exp \frac{\pi}{2} \xi_{i}\right)\right|_{\mathfrak{p}}=\left.\operatorname{ad}_{\xi_{i}}\right|_{\mathfrak{p}}(i=1.2 .3)$, conforme Lema [4.3]. Seja $\left\{X_{1}, J_{1}\left(X_{1}\right), J_{2}\left(X_{1}\right) . J_{3}\left(X_{1}\right), \ldots . X_{n}, J_{1}\left(X_{n}\right), J_{2}\left(X_{n}\right), J_{3}\left(X_{n}\right)\right\}$ base $B$-ortonormal de $\mathfrak{p}$ dada pelo Lema [4.4]. Temos:

$$
\begin{aligned}
K_{i l l}\left(\xi_{i}, \xi_{i}\right)= & \left.\operatorname{traço}\left(a d_{\xi_{i}} \circ a d_{\xi_{i}}\right)\right|_{\mathfrak{p}}+\left.\operatorname{traço}\left(a d_{\xi_{i}} \circ a d_{\xi_{i}}\right)\right|_{\mathfrak{a}_{1}} \\
= & \operatorname{traço}\left(J_{i} \circ J_{i}\right)-8=-\operatorname{traço}\left(I d_{\mathfrak{p}}\right)-8=-4 n-8 \\
= & \operatorname{traço}\left(a d_{\xi_{i}} \circ a d_{\xi_{j}}\right)=\left.\operatorname{traço}\left(a d_{\xi_{i}} \circ a d_{\xi_{j}}\right)\right|_{\mathfrak{p}}+\left.\operatorname{traço}\left(a d_{\xi_{i}} \circ a d_{\xi_{j}}\right)\right|_{\mathfrak{a}_{1}} \\
K_{i l l}\left(\xi_{i}, \xi_{j}\right)= & \left.\operatorname{traço}\left(a d_{\xi_{i}} \circ a d_{\xi_{j}}\right)\right|_{\mathfrak{p}}=\operatorname{traço}\left(J_{i} \circ J_{j}\right)=-\operatorname{traço}\left(J_{j} \circ J_{i}\right) \\
& \Longrightarrow K_{i l l}\left(\xi_{i}, \xi_{j}\right)=0 \operatorname{para} i \neq j \\
K_{i l l}\left(\xi_{i},\left[x_{k}, x_{j}\right]\right)= & K_{i l l}\left(\left[\xi_{i}, x_{k}\right], x_{j}\right)=K_{i l l}\left(J_{i}\left(x_{k}\right), x_{j}\right)=a B\left(J_{i}\left(x_{k}\right), x_{j}\right)=0 \\
K_{i l l}\left(\xi_{i},\left[x_{k}, J_{i}\left(x_{j}\right)\right]\right)= & K_{i l l}\left(\left[\xi_{i}, x_{k}\right], J_{i}\left(x_{j}\right)\right)=K_{i l l}\left(J_{i}\left(x_{k}\right), J_{i}\left(x_{j}\right)\right)=a B\left(J_{i}\left(x_{k}\right), J_{i}\left(x_{j}\right)\right)=0 \\
& \operatorname{para} k \neq j \\
K_{i l l}\left(\xi_{i},\left[x_{k}, J_{i}\left(x_{k}\right)\right]\right)= & K_{i l l}\left(\left[\xi_{i}, x_{k}\right], J_{i}\left(x_{k}\right)\right)=K_{i l l}\left(J_{i}\left(x_{k}\right), J_{i}\left(x_{k}\right)\right)=a B\left(J_{i}\left(x_{k}\right), J_{i}\left(x_{k}\right)\right)=a \\
K_{i l l}\left(\xi_{l},\left[x_{k}, J_{i}\left(x_{k}\right)\right]\right)= & K_{i l l}\left(\left[\xi_{l}, x_{k}\right], J_{i}\left(x_{k}\right)\right)=K_{i l l}\left(J_{l}\left(x_{k}\right), J_{i}\left(x_{k}\right)\right)=a B\left(J_{l}\left(x_{k}\right), J_{i}\left(x_{k}\right)\right)=0 \\
& \operatorname{para} l \neq i
\end{aligned}
$$




$$
\begin{aligned}
& K_{i l l}\left(\xi_{l},\left[x_{k}, J_{i}\left(x_{j}\right)\right]\right)=a B\left(J_{l}\left(x_{k}\right), J_{i}\left(x_{j}\right)\right)=0 \text { para } k \neq j \text { e } l \neq i \\
& K_{i l l}\left(\xi_{l},\left[J_{i}\left(x_{k}\right), J_{s}\left(x_{k}\right)\right]\right)=K_{i l l}\left(\left[\xi_{l}, J_{i}\left(x_{k}\right)\right], J_{s}\left(x_{k}\right)\right)=K_{i l l}\left(\left(J_{l} \circ J_{i}\right)\left(x_{k}\right), J_{s}\left(x_{k}\right)\right) \\
& = \begin{cases} \pm K_{i l l}\left(J_{s}\left(x_{k}\right), J_{s}\left(x_{k}\right)\right)= \pm a B\left(J_{s}\left(x_{k}\right), J_{s}\left(x_{k}\right)\right)= \pm a & \text { para } l \neq s \neq i \\
K_{i l l}\left(\left(J_{i} \circ J_{i}\right)\left(x_{k}\right), J_{s}\left(x_{k}\right)\right)=-a B\left(x_{k}, J_{s}\left(x_{k}\right)\right)=0 & \text { para } l=i \\
z \text { zero } & \text { caso contrário }\end{cases}
\end{aligned}
$$

Assim

$$
\begin{aligned}
{\left[x_{k}, J_{i}\left(x_{k}\right)\right] } & \equiv \frac{a}{-4 n-8} \xi_{i} \bmod l_{1} \quad \text { para } i=1,2,3 \\
{\left[J_{i}\left(x_{k}\right), J_{s}\left(x_{k}\right)\right] } & \equiv \pm \frac{a}{-4 n-8} \xi_{l} \bmod l_{1} \quad \text { para } i \neq s \neq l
\end{aligned}
$$

Portanto, $\Theta_{X}: \mathfrak{p} \rightarrow \mathfrak{h} / \mathfrak{l}_{1}$ é sobrejetora $\forall X \in \mathfrak{p}$, e isto conclui a demonstração que $\left(\mathfrak{g}_{u}, s, \mathfrak{l}_{1}, B\right)$ é, de fato, uma LISO-álgebra. Finalmente, a irredutibilidade de $\left(\mathfrak{g}_{u}, s, \mathfrak{l}_{1}, B\right)$ é consequência direta da simplicidade de $\mathfrak{g}_{u}$.

Corolário 4.1 Seja $G_{u} / H$ um espaço de Wolf quaterniônico, simplesmente conexo, compacto (portanto irredutícel). Então existe um fibrado canônico $G_{u} / L_{1}$ sobre $G_{u} / H$ com fibras $H / L_{1}$ difeomorfas à esfera $S p(1)$, e que possui uma estrutura de espaço sub-simétrico, simplesmente conexo, compacto, irredutivel.

\section{Prova:}

Considera-se a ALIO quaterniônica compacta irredutível $\left(\mathfrak{g}_{u}, s, B\right)$ correspondente a $G_{u} / H$, decompõe-se $\mathfrak{g}_{u}=\mathfrak{h} \oplus \mathfrak{p}$ sob $s$, com $\mathfrak{h}=\mathfrak{l}_{1} \oplus \mathfrak{a}_{1}, \mathfrak{a}_{1} \cong \mathfrak{s p}(1)$ e obtém-se a LISO-álgebra irredutivel associada $\left(\mathfrak{g}_{u}, s, \mathfrak{I}_{1}, B\right)$, como na Proposição [4.2]. O espaço sub-simétrico compacto construido a partir de $\left(\mathfrak{g}_{u}, s, \mathfrak{l}_{1}, B\right)$ é $G_{u} / L_{1}$, onde $L_{1}=\exp \mathfrak{l}_{1}$, a distribuição sobre $G_{u} / L_{1}$ é a $G_{u}$-distribuição determinada no ponto base por p e a métrica sobre a distribuição é a métrica $G_{u}$-invariante determinada no ponto base por $B$ (neste caso $B$ é um múltiplo negativo da forma de Killing de $\mathfrak{g}_{u}$ ). Agora $H / L_{1} \rightarrow G_{u} / L_{1} \rightarrow G_{u} / H$ é um fibrado, cuja fibra típica $H / L_{1}$ é difeomorfa à $S p(1)$, pois $\mathfrak{h} / \mathfrak{l}_{1} \cong \mathfrak{a}_{1} \cong \mathfrak{s p}(1)$. 
Observamos que em analogia com a teoria de álgebras de Lie involutivas ortogonais, a cada LISO-álgebra ( $\mathfrak{g}, s, \mathfrak{k}, B) \cdot \mathfrak{g}=\mathfrak{h} \oplus \mathfrak{p}$ sob $s$, corresponde uma LISO-álgebra dual $\left(\mathfrak{g}^{\times}, s^{\star}, \mathfrak{k}, B^{\times}\right)$ onde $\mathfrak{g}^{\times}=\mathfrak{h} \oplus \mathfrak{p}^{\times} \operatorname{sob} s^{\times} \cdot \mathfrak{p}^{\times}=i \mathfrak{p} ; s^{\times}(h+i p)=s(h)+i s(p) \forall h+i p \in \mathfrak{g}^{\times} ; B^{\star}(X . Y)=B(i X, i Y)$ $\forall \mathrm{X}, Y \in \mathfrak{p}^{*}$. Além disso. $(\mathfrak{g}, s, \mathfrak{k}, B)$ é irredutível se, e somente se $\left(\mathfrak{g}^{\times}, \mathfrak{s}^{\times} . \mathfrak{k}, B^{\times}\right)$é irredutível. Também, se $\left(\mathfrak{g}^{*}, s^{*}, \mathfrak{k}, B^{\times}\right)$é irredutível então exatamente uma entre $\mathfrak{g}$ e $\mathfrak{g}^{*}$ é compacta. Uma demonstração destas afirmaçòes, no contexto de ALIO's, se encontra em ([1i]. Lema \$.2.6), e pode ser transcrita igualmente aqui, no contexto de LISO-álgebras.

Feitas estas ponderações. a cada espaço de Wolf quaterniônico, compacto, simplesmente conexo, corresponde precisamente um par dual de espaços sub-simétricos, simplesmente conexos, irredutíveis (um compacto e outro nào compacto).

\section{EXEMPLOS:}

Da classificação dos espaços de Wolf quaterniônicos, simplesmente conexos, compactos. irredutíveis ([9], tabela 5. î), organizamos abaixo a lista dos espaços sub-simétricos irredutiveis compactos, construidos como no corolário acima, e de seus duais não compactos:

$$
\begin{array}{rc}
S U(n+2) / S(U(1) \times U(n)) & S U(n, 2) / S(U(1) \times U(n)) \\
S O(n+4) / S O(n) \times\left(S p(1) / Z_{2}\right) & S O_{e}(n, 4) / S O(n) \times\left(S p(1) / Z_{2}\right) \\
S p(n+1) / S p(n) & S p(n+1, I R) / S p(n) \\
E_{6} /\left(S U(6) / Z_{2}\right) & E_{6}^{*} /\left(S U(6) / Z_{2}\right) \\
E_{8} /\left(E_{7} / Z_{2}\right) & E_{8}^{*} /\left(E_{i} / Z_{2}\right) \\
F_{4} /\left(S p(3) / Z_{2}\right) & F_{4}^{*} /\left(S p(3) / Z_{2}\right) \\
G_{2} /\left(S p(1) / Z_{2}\right) & C_{2}^{* *} /\left(S p(1) / Z_{2}\right)
\end{array}
$$

Para ilustrar a tabela acima, descreveremos agora, em detalhes, a estrutura sub-simétrica de algumas variedades sub-Riemannianas referidas nesta lista. Denotemos por $E^{i, j}$ a matriz quadrada com um na $(i, j)$-ésima posição e zero nas demais.

\section{O espaço sub-simétrico $S p(2) / S p(1)$}

A álgebra de Lie $\mathfrak{g}_{u}=\mathfrak{s p}(2)$ possui uma estrutura de LISO-álgebra dada por:

$$
\mathfrak{s p}(2)=\mathfrak{h} \oplus \mathfrak{p} \operatorname{sob} s, \quad \mathfrak{h}=\mathfrak{l}_{1} \oplus \mathfrak{a}_{1}
$$


onde

$$
\begin{gathered}
\mathfrak{l}_{1}=\mathfrak{s p}(1)=\left\{\left(\begin{array}{cccc}
0 & 0 & 0 & 0 \\
0 & i a_{1} & 0 & b_{1}+i c_{1} \\
0 & 0 & 0 & 0 \\
0 & -b_{1}+i c_{1} & 0 & -i a_{1}
\end{array}\right) \in g l(4, \mathbb{C}): a_{1}, b_{1}, c_{1} \in \mathbb{R}\right\} \\
\mathfrak{a}_{1}=\mathfrak{s p}(1)=\left\{\left(\begin{array}{cccc}
i a_{2} & 0 & b_{2}+i c_{2} & 0 \\
0 & 0 & 0 & 0 \\
-b_{2}+i c_{2} & 0 & -i a_{2} & 0 \\
0 & 0 & 0 & 0
\end{array}\right) \in g l(4, \mathbb{C}): a_{2}, b_{2}, c_{2} \in \mathbb{R}\right\} \\
\mathfrak{p}=\mathbb{R}^{4}=\left\{\left(\begin{array}{cccc}
-a_{3}+i b_{3} & 0 & a_{4}+i b_{4} & 0 \\
0 & -a_{4}+i b_{4} & 0 & a_{3}-i b_{3} \\
0 & 0 & -a_{3}-i b_{3} & 0
\end{array}\right) \in g l(4, \mathbb{C}): a_{3}, b_{3}, a_{4}, b_{4} \in \mathbb{R}\right\}
\end{gathered}
$$

A involução $s$ sobre $\mathfrak{g}_{u}=\mathfrak{s} \mathfrak{p}(2)$ é dada por:

$$
\begin{aligned}
s=A d_{\psi}: \mathfrak{s p}(2) & \rightarrow \mathfrak{s p}(2) \\
A & \mapsto \psi \cdot A . \psi
\end{aligned} \quad \text { onde } \quad \psi=\left(\begin{array}{cccc}
-1 & 0 & 0 & 0 \\
0 & 1 & 0 & 0 \\
0 & 0 & -1 & 0 \\
0 & 0 & 0 & 1
\end{array}\right)
$$

Uma base de $\mathfrak{p}$ é: $\gamma=\left\{X_{1}=-E^{1,2}+E^{2.1}-E^{3,4}+E^{4,3}, X_{2}=i\left(E^{1,2}+E^{2,1}-E^{3,4}-\right.\right.$ $\left.\left.E^{4,3}\right), \quad X_{3}=E^{1,4}+E^{2,3}-E^{3,2}-E^{4,1}, X_{4}^{\prime}=i\left(E^{1,4}+E^{2,3}+E^{3,2}+E^{4,1}\right)\right\}$

As possíveis métricas $B$ definidas sobre $\mathfrak{p}, a d_{\mathfrak{l}_{1}}$-invariantes, são tais que:

$$
B\left(X_{i}, X_{i}\right)=a \in \mathbb{R}_{+}^{*} \quad \text { para } i=1,2,3,4 \quad B\left(X_{i}, X_{j}\right)=0 \quad \text { para } i \neq j
$$

A álgebra quaterniônica ortogonal sobre $\mathfrak{p}$ é gerada pela base quaterniônica: $\left\{I d, J_{1}, J_{2}\right.$, $\left.J_{3}\right\}$ onde $J_{k}=\left.a d_{\xi_{k}}\right|_{\mathfrak{p}}(\mathrm{k}=1,2,3), \quad \xi_{1}=i\left(E^{1,1}-E^{3,3}\right), \quad \xi_{2}=E^{1,3}-E^{3,1}, \quad \xi_{3}=i\left(E^{1,3}+E^{3,1}\right)$.

Um cálculo simples, mostra que $\gamma$ é base quaterniônica de $\mathfrak{p}$, pois: $X_{2}=J_{1}\left(X_{1}\right), X_{3}=$ $J_{2}\left(X_{1}\right), X_{4}=J_{3}\left(X_{1}\right)$. 
O espaço sub-simétrico $S U(n+2) / S(U(1) \times U(n))$

A álgebra de Lie $\mathfrak{g}_{u}=\mathfrak{s u}(n+2)$ possui uma estrutura de LISO-álgebra dada por:

$$
\mathfrak{s u}(n+2)=\mathfrak{h} \oplus \mathfrak{p} \text { sob } s, \quad \mathfrak{h}=\mathfrak{l}_{1} \oplus \mathfrak{a}_{1}
$$

onde

$$
\begin{aligned}
& \mathfrak{h}=\mathfrak{s}(\mathfrak{u}(2)+\mathfrak{u}(n))=\left\{\left(\begin{array}{cc}
A & \\
& B
\end{array}\right) \in g l(n+2, \mathbb{C}): \begin{array}{c}
A \in \mathfrak{u}(2), B \in \mathfrak{u}(n) . \\
\operatorname{traço} A+\operatorname{traço} B=0
\end{array}\right\} \\
& \mathfrak{l}_{1}=\mathfrak{s}(\mathfrak{u}(1)+\mathfrak{u}(n))=\left\{\left(\begin{array}{ccc}
i a & 0 \\
0 & i a & \\
& & B
\end{array}\right) \in g l(2+n, \mathbb{C}): \begin{array}{c}
B \in \mathfrak{u}(n), a \in \mathbb{R}, \\
\operatorname{traço} B+2 i a=0
\end{array}\right\}
\end{aligned}
$$$$
\mathfrak{a}_{1}=\mathfrak{s p}(1)=\left\{\left(\begin{array}{cc}
i a_{1} & b_{1}+i c_{1} \\
-b_{1}+i c_{1} & -i a_{1} \\
&
\end{array}\right) \in \operatorname{gl}(n+2, \mathbb{C}): a_{1}, b_{1}, c_{1} \in \mathbb{R}\right\}
$$

$$
\mathfrak{p}=\mathbb{R}^{4 n}=\left\{\left(\begin{array}{ccccccc}
0 & 0 & z_{1} & z_{2} & z_{3} & \ldots & z_{n} \\
0 & 0 & w_{1} & w_{2} & w_{3} & \ldots & w_{n} \\
-\overline{z_{1}} & -\bar{w}_{1} & & & & & \\
-\overline{z_{2}} & -\bar{w}_{2} & & & & \\
-\overline{z_{3}} & -\bar{w}_{3} & & & 0 & \\
& & & &
\end{array}\right) \in g l(2+n, \mathbb{C}): \begin{array}{c}
z_{i}, w_{i} \in \mathbb{C}^{\prime} \\
(i=1,2, \ldots, n)
\end{array}\right\}
$$


A involução s sobre $\mathfrak{g}_{u}=\mathfrak{s u}(n+2)$ é dada por:

$$
\begin{aligned}
s=A d_{\psi}: \mathfrak{s u}(n+2) & \rightarrow \mathfrak{s u}(n+2) \\
A & \mapsto \psi \cdot A \cdot \psi
\end{aligned} \quad \text { onde } \quad \iota \cdot=\left(\begin{array}{ccc}
-1 & 0 & \\
0 & -1 & \\
& & \\
& & I d_{n \times n}
\end{array}\right)
$$

Uma base de p é: $\gamma=\left\{X_{k}=E^{1, k+2}-E^{k+2.1}, \quad Y_{k}=i\left(E^{1, k+2}+E^{k+2,1}\right), Z_{k}=E^{k+2.2}-\right.$ $\left.E^{2, k+2}, \quad D_{k}=i\left(E^{2, k+2}+E^{k+2,2}\right), k=1,2, \ldots, n\right\}$

Um produto interno $B$ definido sobre $\mathfrak{p}, a d_{\mathfrak{l}_{1}}$-invariante, pode ser dado por:

$B(\cdot, \cdot)=\left.a K_{i l l}\right|_{\mathfrak{p}_{\times} \mathfrak{p}}(\cdot, \cdot) \quad(a<0 \in \mathbb{R}), \quad$ onde $\quad K_{i l l}(X, Y)=2(n+2) \operatorname{traço}(X Y) \quad$ é a forma de Killing de $\mathfrak{s u}(n+2)$.

A álgebra quaterniônica ortogonal sobre $\mathfrak{p}$ é gerada pela base quaterniônica: $\left\{I d, J_{1}, J_{2}\right.$, $\left.J_{3}\right\}$ onde $J_{k}=\left.a d_{\xi_{k}}\right|_{\mathfrak{p}}(\mathrm{k}=1,2,3), \quad \xi_{1}=i\left(E^{1,1}-E^{2,2}\right), \quad \xi_{2}=E^{1,2}-E^{2,1}, \quad \xi_{3}=i\left(E^{1,2}+E^{2,1}\right)$.

Um cálculo simples, mostra que $\gamma$ é base quaterniônica de $\mathfrak{p}$, pois: $Y_{k}=J_{1}\left(X_{k}\right), Z_{k}=$ $J_{2}\left(X_{k}\right), D_{k}=J_{3}\left(X_{k}\right)$. 


\section{Bibliografia}

[1] Bieliavsky, P., Falbel. E. and Gorodski, C. : The classification of simply-connected contact sub-Riemannian symmetric spaces, Pacific J. Math. 188 (1) (1999), 65-\$1.

[2] Falbel E. and Gorodski C. : On contact sub-Riemannian symmetric spaces. Ann. Sc. Éc. Norm. Sup. 28 (4) (1995). 5i1-589.

[3] Falbel E. and Gorodski C. : Sub-Riemannian homogeneous spaces in dimensions 3 and 4. Geometriae Dedicata 62 (3) (1996), 227-252.

[4] Falbel E., Gorodski C. and Rumin M. :Holonomy of sub-Riemannian manifolds, International Journal of Mathematics 8 (3) (1997), 317-344.

[5] Falbel E., Verderesi J.A. and Veloso J.M. : The equivalence problem in sub-Riemannian geometry, Preprint IME-USP. 1993.

[6] Helgason, S. : Differential geometry, Lie groups, and symmetric spaces, Academic Press, 1978.

[i] Kobayashi, S. and Nomizu, K. : Foundations of differential geometry, Wiley Interscience Publishers, 1963-1969.

[8] Montgomery, R. : A survey of singular curves in sub-Riemannian geometry, J. Dyn. and Control Syst. 1 (1) (1995). 49-90.

[9] Salamon, S. : Riemannian geometry and holonomy groups, Longman Scientific and Technical, 1989.

[10] Strichartz, R.S. : Sub-Riemannian geometry, J. Differential Geom. 24 (1986), 221-263.

[11] Strichartz, R.S. : Corrections to "Sub-Riemannian geometry", J. Differential Geom. 30 (1989), 595-596. \& (3) (1997) 317-344.

[12] Tausk, D. : Regularidade de curvas minimizantes em espaços sub-Riemannianos homogêneos, dissertação de mestrado IME-USP, 1998. 
[1:3] Hamenstäd, U. : Some regularity theorems for Carnot-Caratheodory metrics. J. Differential Geom. 32 (1990) \$19-\$50.

[14] Varadarajan, V. S. : Lie groups, Lie algebras and their representations. Springer-Verlag, New York, 1984.

[15] Warner, F. W. : Foundations of differentiable manifolds and Lie groups. Glenview, Scott, 1971.

[16] Wolf, J. A. : Complex homogeneous contact manifolds and quaternionic symmetric spaces, J. Math. Mech. 14 (6) (1965), 1033-1047.

[17] Wolf. J. A. : Spaces of constant curvature, Publish or Perish, Inc., 1984. 